\title{
Les occupations néolithiques de Démouville « ZAC du Clos Neuf » (Calvados) et les débuts de la colonisation agricole en Normandie
}

The Neolithic occupations of Démouville "ZAC du Clos Neuf" (Calvados) and the beginning of the spread of agriculture in Normandy

Las ocupaciones neolíticas de Démouville "ZAC du Clos Neuf" (Calvados) y los inicios de la colonización agrícola en Normandía Die neolithischen Siedlungsfundstellen von Démouville "ZAC du Clos Neuf" (Calvados) und die Anfänge der landwirtschaftlichen Besiedlung in der Normandie

Maud Le Saint-Allain, Cyrille Billard, François Charraud, Marie-France Dietsch-Sellami et Denis Jan

\section{OpenEdition} Journals

Édition électronique

URL : https://journals.openedition.org/rao/7239

DOI : $10.4000 /$ rao.7239

ISSN : $1775-3732$

Éditeur

Presses universitaires de Rennes

Édition imprimée

Date de publication : 21 décembre 2021

Pagination : 41-77

ISBN : 978-2-7535-8668-0

ISSN : 0767-709X

Référence électronique

Maud Le Saint-Allain, Cyrille Billard, François Charraud, Marie-France Dietsch-Sellami et Denis Jan, «Les occupations néolithiques de Démouville «ZAC du Clos Neuf » (Calvados) et les débuts de la colonisation agricole en Normandie », Revue archéologique de l'Ouest [En ligne], 37 | 2021, mis en ligne le 21 décembre 2021, consulté le 11 octobre 2022. URL : http://journals.openedition.org/rao/7239 ; DOI : https://doi.org/10.4000/rao.7239 


\title{
Les occupations néolithiques de Démouville « ZAC du Clos Neuf » (Calvados) et les débuts de la colonisation agricole en Normandie
}

\author{
The Neolithic occupations of Démouville "ZAC du Clos Neuf" (Calvados) and the beginning of \\ the spread of agriculture in Normandy
}

Maud Le Saint-Allain

Inrap Grand-Ouest centre de Carquefou, 4 rue du Tertre 44470 Carquefou

Cyrille Billard

DRAC Normandie - service régional de l'archéologie, 13 bis rue Saint-Ouen 14000 Caen, CreAAH, Rennes-UMR 6566

François Charraud

Inrap, 4 boulevard de l'Europe 14540 Bourguébus

Marie-France Dietsch-Sellami

Inrap GSO, Domaine de Campagne, Pôle mixte de Recherches 24260 Campagne, ISEM - Institut des Sciences de l'Évolution de Montpellier, UMR 5554 équipe Dynamique de la biodiversité, anthropoécologie

Denis Jan

Service archéologie du Calvados, 36 rue Fred-Scamaroni 14000 Caen

Résumé : Outre une nécropole de l'âge du Bronze, la fouille préventive du site de Démouville "ZAC du Clos Neuf » (Calvados) a permis la mise au jour de structures néolithiques, dont une bonne partie pourrait correspondre à des structures de stockage. L'analyse du mobilier et les datations radiocarbone ont conduit à individualiser deux occupations principales : l'une au début du Néolithique ancien et la seconde au Néolithique moyen.

Le mobilier céramique de l'occupation du Néolithique ancien de Démouville, bien que peu abondant, permet de rattacher les structures les plus anciennes à un petit groupe de sites normands attribués à une étape finale du RRBP. Par ses datations convergentes avec celles de Colombelles, le site de Démouville assure un bon ancrage chronologique à cette phase pionnière et confirme la présence de la céramique non rubanée de type Limbourg dans le contexte rubané de Normandie. La découverte de deux blocs d'hématite et d'un grand pic en grès confirme également l'intégration précoce de ce matériau colorant dans les réseaux d'échange des pionniers rubanés et peut constituer un indice fort de l'émergence d'une activité d'extraction minière. Les structures du Néolithique ancien sont ici associées à un groupe d'au moins six fosses du Néolithique moyen qui forment un type de sites assez récurrents dans la Plaine de Caen et qui semblent voués à la transformation et au stockage des produits des récoltes.

Démouville constitue enfin une référence par l'abondance de macrorestes végétaux découverts (pour les deux périodes d'occupation néolithiques). Pour le Néolithique ancien, si le site s’inscrit bien dans les pratiques agraires rubanées, il s’en démarque (comme à Colombelles), par l'abondance de l'orge vêtue, qui est seulement observée dans les contextes rubanés à l'est du Rhin. La culture du blé nu vient enfin à nouveau alimenter le débat d'une possible introduction de cette céréale depuis les régions méditerranéennes et d'échanges à longue distance précoces avec la sphère cardiale.

Pour le Néolithique moyen, la prépondérance de l'orge nue, et plus généralement des céréales à grains nus, rejoint les observations formulées à propos des contextes de la moitié nord de la France.

Abstract: In complement to the discovery of a Bronze Age necropolis, the preventive excavations at Démouville " $Z A C$ du Clos Neuf" (Calvados) unearthed Neolithic features, probably storage structures. The analysis of the archaeological material defined two main phases of occupation, the first dating to the Early Neolithic, the second to the Middle Neolithic. 
The Early Neolithic pottery is not abundant, but links the earlier features to a small group of sites in Normandy that are dated to the final phase of the "Rubané Récent du Bassin Parisien". Contemporary to the site at Colombelles, Démouville forms a sound chronological basis for this early phase and highlights the presence of the non rubanée Limbourg pottery in the Rubané context of Normandy. The discovery of two hematite blocks and a sandstone pick also confirms the early presence of this pigment in the exchange networks of the Rubané pioneers and constitutes proof for the emergence of mineral extraction activity in the area.

The Démouville site also includes a group of at least six pits dating to the Middle Neolithic, of a recurrent type in the "Plaine of Caen " area and were probably used for transforming and storing crops.

The abundant botanical macroremains (for both occupation phases) are a reference point. For the Early Neolithic, the site adopts the usual Rubane land use practices, but differs (as at Colombelles), by the abundance of barley, which is only observed in the Rubane contexts east of the Rhine valley. The cultivation of naked wheat feeds the debate on the possible introduction of this cereal from the Mediterranean regions and on early long-distance exchanges with the "Cardial" area.

For the Middle Neolithic, the preponderance of naked barley, and more generally of naked cereals, is consistent with observations about the North half France contexts.

Mots clés : Démouville, Normandie, Néolithique, Rubané, graines, restes végétaux.

Keywords: Démouville, Normandy, Neolithic, Rubané, seeds, vegetal remains.

Mise à part une importante nécropole de l'âge du Bronze qui a déjà donné lieu à une publication (Le SaintAllain et al., 2013), le site de Démouville a livré des vestiges néolithiques qui, bien que modestes, n'en livrent pas moins des informations remarquables sur la néolithisation de la partie normande du Bassin parisien. Son intérêt est accru par la proximité du site de Colombelles dont le rattachement à la sphère rubanée et plus particulièrement à une étape contemporaine de la fin du Rubané récent du Bassin parisien ou du début du groupe de VilleneuveSaint-Germain a été récemment mis en évidence (Billard et al., 2014).

Outre le cumul de plusieurs occupations du Néolithique ancien et du Néolithique moyen, ce site offre la particularité d'avoir livré de nombreuses structures ayant pu avoir une fonction de stockage et de nombreux macrorestes végétaux documentant les premières pratiques agraires.

L'accent sera donc ici porté sur ces occupations néolithiques dont l'imbrication entre Néolithique ancien et moyen mérite d'être analysée.

\section{CirCONSTANCES DE L'OPÉRATION}

La réalisation d'un diagnostic archéologique par François Delahaye (Inrap) en 2006 a permis la mise au jour de vestiges d'habitat attribués au Néolithique sans précision ainsi qu'une nécropole non datée à ce stade, mais pour laquelle il était alors proposé une attribution au Néolithique ou à la protohistoire. Le site néolithique n'était représenté que par quelques fosses dont l'une avait toutefois livré un grand nombre de céréales carbonisées.
La société SHEMA, aménageur de la ZAC du Clos Neuf pour le compte de la commune de Démouville, fit réaliser la fouille en 2010 par la société Oxford Archéologie sous la direction de Maud Le Saint-Allain sur une emprise de $6240 \mathrm{~m}^{2}$ (fig. 1 et 2).

À l'issue de la fouille et du lavage du mobilier, une grande fosse située en limite occidentale d'emprise s'est révélée appartenir à la culture rubanée, conduisant le service régional de l'archéologie à envisager une extension de l'opération vers l'ouest (conduite sous la responsabilité de C. Billard). La présence des remblais de la fouille, stockés en merlon, a compliqué cette extension, mais un complément de décapage a permis d'étendre l'aire d'étude d'environ $1100 \mathrm{~m}^{2}$, soit une surface totale de fouille de $7340 \mathrm{~m}^{2}$ (fig. 2). Aucune nouvelle fosse ni aucun trou de poteau pouvant être rattaché à l'occupation néolithique n'a pu être identifié dans l'emprise de cette extension.

\section{LE CADRE GÉOGRAPHIQUE ET GÉOLOGIQUE}

Le site de Démouville est localisé dans la Plaine de Caen, à l'est de l'agglomération caennaise, à une dizaine de kilomètres de la côte (fig. 1). Malgré la mise en culture de la parcelle, aucune découverte de surface ne laissait présager la présence de vestiges archéologiques.

Le site est bordé au nord-ouest par l'estuaire de l'Orne et la vallée de la Dives à l'est. À l'échelle locale, les variations d'altitude sont très peu marquées. Deux tiers de l'emprise fouillée se caractérisent par une légère dépression orientée sud-ouest - nord-est qui matérialise l'amorce de la vallée de la Gronde. La zone d'étude occupe un vaste plateau de calcaires bathoniens faiblement incli- 

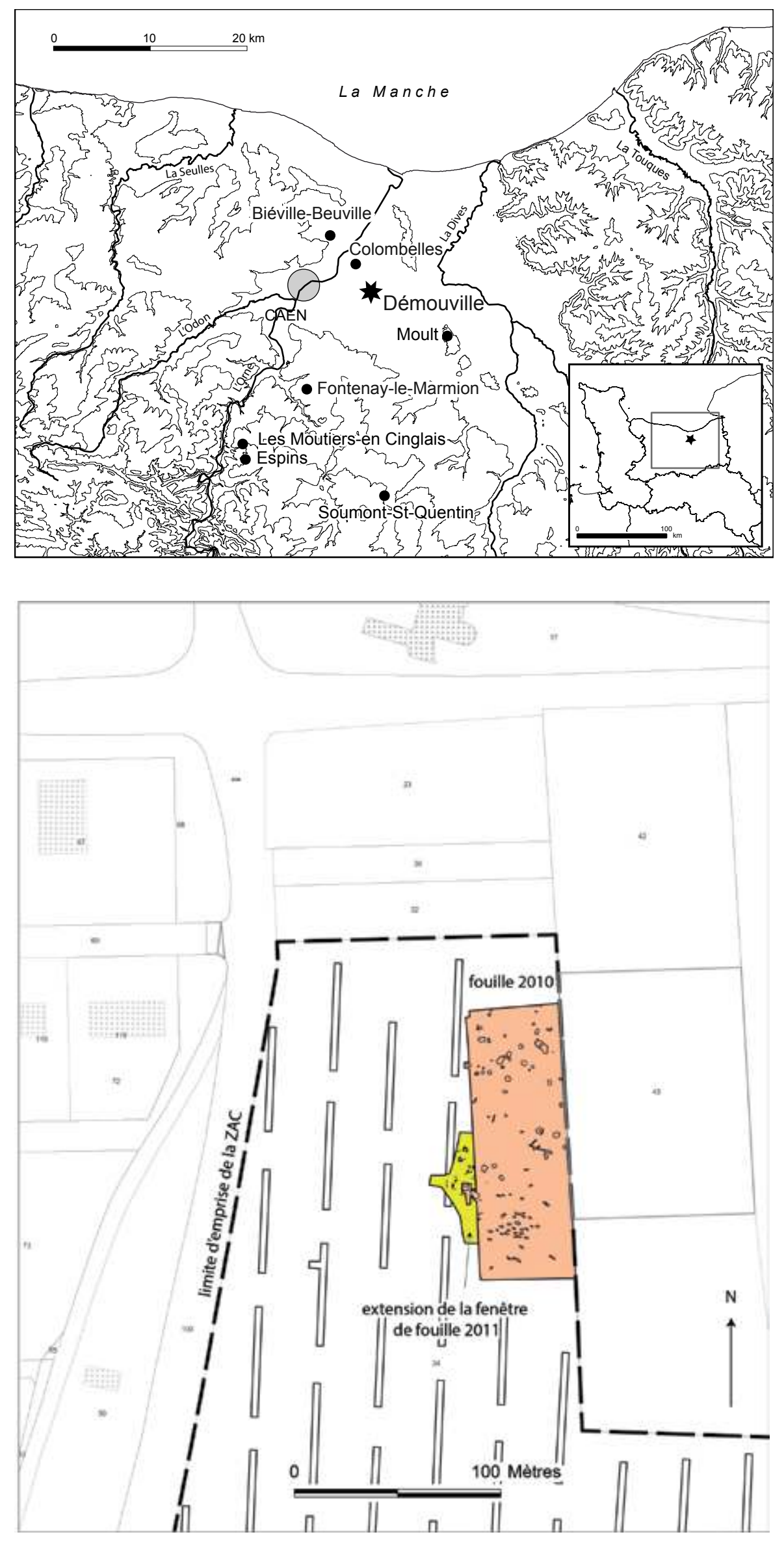

Figure 1 : Situation du site de Démouville et des principaux sites nommés dans le texte.

Figure 1: Location of the Démouville site and other principal sites named in the text.
Figure 2 : Plan de l'emprise de la fouille.

Figure 2: Plan of the excavation. 
nés vers l'est-nord-est, à une altitude située entre 46 et $52 \mathrm{~m}$. Ce plateau, traditionnellement dévolu aux grandes cultures céréalières et industrielles, est recouvert de limons quaternaires en placages dont l'épaisseur augmente en se rapprochant du littoral de la Manche. Ces loess fortement carbonatés offrent un profil de sols bruns décarbonatés en surface tout à fait comparables à ceux observés sur le site de Colombelles et peuvent dépasser $2 \mathrm{~m}$ d'épaisseur.

\section{POSITION DE LA NÉCROPOLE DE L'ÂGE DU BRONZE PAR RAPPORT AUX VESTIGES NÉOLITHIQUES (FIG. 3 ET 4)}

D'une manière générale, les vestiges néolithiques sont localisés en limite ouest de l'emprise et dans sa partie nord. La nécropole de l'âge du Bronze occupe la partie sud de l'emprise et se partage entre des inhumations et des enclos circulaires partiels (Le Saint-Allain et al., 2013; Le Saint-Allain, 2013). Elle a livré quatre enclos circulaires partiels auxquels s'ajoutent des tronçons de fossés qui rappellent une organisation semblable à celle de la nécropole de Mondeville (Chancerel et al., 2006). Les fossés sont très peu marqués : en moyenne, une trentaine de centimètres de large pour une vingtaine de centimètres de profondeur conservée. Le nombre de sépultures s'élève à 50,45 inhumations attestées et 5 fosses vides de restes osseux. Signalons que l'ensemble de la zone a donné lieu à un décapage complémentaire, qui a d'ailleurs permis de reconnaître trois de ces sépultures.

Létat de conservation des squelettes est globalement médiocre. Parmi les 45 individus, 30 sont préservés à moins de $30 \%$ et seul un individu est complet. Les données biologiques en sont restreintes.

La plupart des sépultures offre un mode de décomposition en espace semi-colmaté et quelques effets de contrainte ont pu être observés en dehors des parois de la fosse. L'orientation des sépultures est assez hétérogène. De la même façon, les positions des individus sont très variées.

Seules trois sépultures ont livré un mobilier d'accompagnement constitué d'un collier de dentales (sép. 83), de deux perles en pierre (sép. 111) et de quatre perles en ambre (sép. 97).

Les datations ${ }^{14} \mathrm{C}$ (tabl. 8) s'échelonnent entre la fin du Bronze ancien et le Bronze moyen.

\section{LES VESTIGES NÉOLITHIQUES}

La particularité du site de Démouville vient de la superposition sur le même emplacement de quatre structures rattachables au premier front de colonisation agricole et de six structures appartenant au Néolithique moyen. Six autres structures néolithiques, non datées précisément, viennent compléter cet ensemble caractérisé par l'absence de restes fauniques, mais par une bonne conservation des macrorestes végétaux.

Face à un faible corpus céramique, la seule méthode pour essayer de distinguer les différentes occupations a consisté à multiplier les datations par radiocarbone, réalisées le plus souvent sur des carporestes à durée de vie courte.

\section{Une nouvelle occupation rubanée dans la Plaine de Caen (st. 26, 28, 86, 174)}

\section{Description des structures (fig. 5)}

\section{- La structure 86 (fig. 5 à 7 )}

La structure 86 est une grande fosse rubanée à remplissage limoneux brun sombre. De forme oblongue, elle atteint 4,80 $\mathrm{m}$ de long pour une largeur de $1,80 \mathrm{~m}$ et une profondeur conservée de $0,52 \mathrm{~m}$. Elle respecte une orientation nord-ouest/sud-ouest, caractéristique de ce type d'habitat. Deux comblements principaux ont été observés : le comblement supérieur (US 161.1 et 161.2) consiste en un limon argileux brun grisâtre, assez meuble et présentant des inclusions charbonneuses. Il recouvre une matrice limono-argileuse constituée d'un limon brun jaunâtre, à rares particules charbonneuses. Le mobilier abondant qui en est issu (céramique, industrie lithique, bloc d'hématite) renvoie aux productions du Rubané (voir infra) et n'a pas montré de répartition préférentielle au sein de la fosse.

Une datation par radiocarbone AMS, effectuée sur noisette carbonisée (tabl. 8) a livré le résultat suivant : Lyon7798(GrA) : [5214 à 4947] av. J.-C. cal.

\section{- La structure 174 (fig. 5)}

La structure 174, grande fosse rubanée à remplissage limoneux brun sombre, se situe dans le même alignement que la précédente et présente les mêmes caractéristiques de comblement et de profil. Son extrémité ouest a livré quelques charbons de bois (dont un carporeste), quelques esquilles de silex et une armature danubienne. Le mobilier y a été retrouvé en bien moindre abondance, mais il est conforme à celui de la fosse 86 . Cette fosse a fait elle aussi l'objet d'une datation ${ }^{14} \mathrm{C}$ sur carporeste carbonisé livrant un intervalle très similaire au précédent : Lyon-8302 : [5207-4947] av. J.-C. cal.

\section{- La fosse 26 (fig. 5 et 8)}

La structure 26 est éloignée d'une soixantaine de mètres au nord-est des deux fosses précédentes. Elle se 
structures rattachables au Néolithique ancien

structures rattachables

au Néolithique moyen

structures probablement

néolithiques

structures rattachables

à l'âge du Bronze (nécropole)

sépultures de l'âge du Bronze

structures Seconde

Guerre mondiale

structures non datées

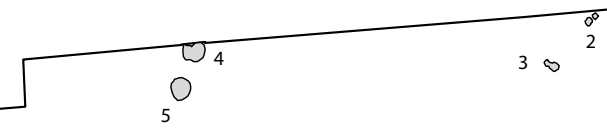

$\stackrel{\circ}{2} 1$

i 7

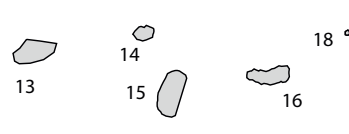

$\bigodot_{9}^{8} 8_{11}^{10}$

$\mathrm{O}_{12}$

$0_{0}^{178}{ }_{38}{ }_{33}^{\circ} \overbrace{167}^{26} \int_{31}^{150}$

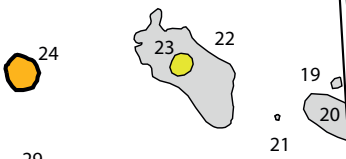

$\infty_{30}^{29}$

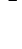

20

$0^{0} \mathrm{O}_{34 / 103}$

$28 \quad 27$

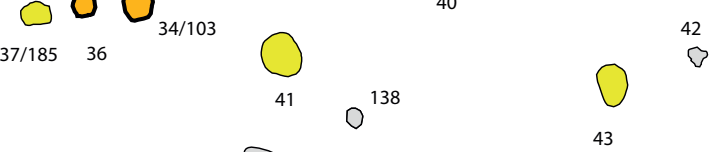

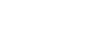

$\bigcirc$

${ }_{139} \circ \int_{152} 0_{137}$

${ }_{46}^{\circ} \int_{47} \quad{ }_{160}{ }_{0^{\circ}}^{0^{49}}{ }^{159}$

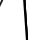

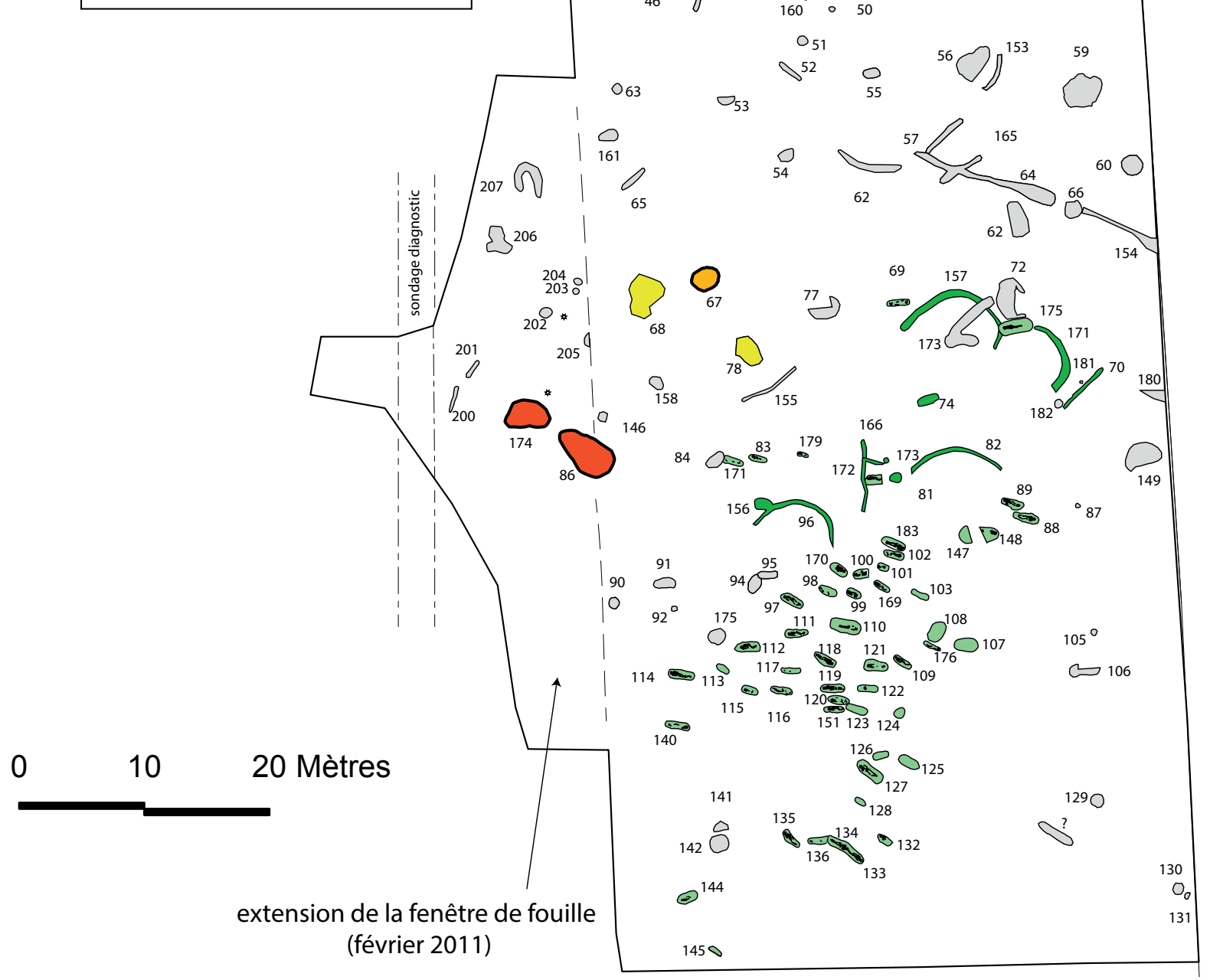

Figure 3 : Plan général phasé des structures.

Figure 3: General plan of the features by phase. 


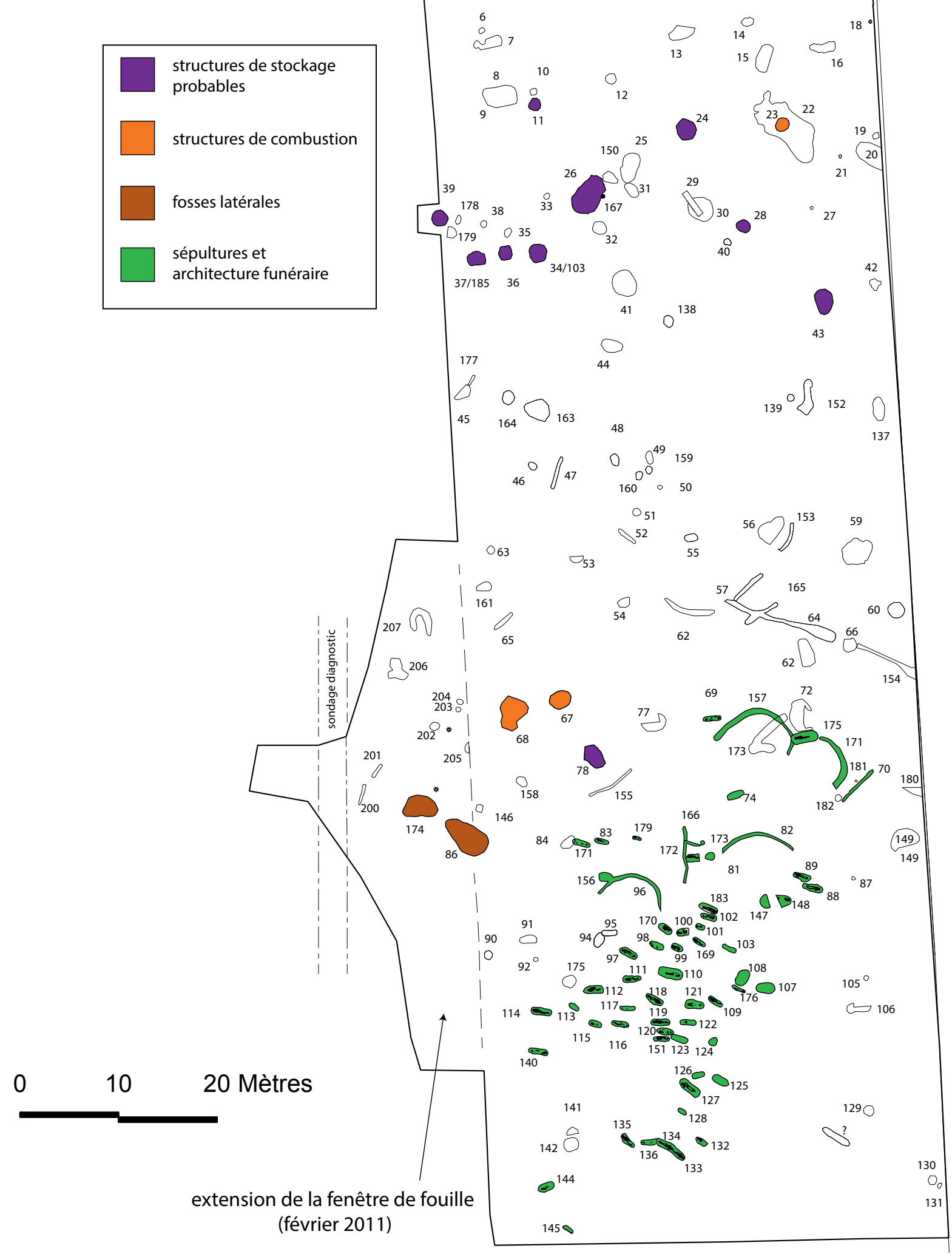

Figure 4 : Plan général des structures par types fonctionnels.

Figure 4: General plan of the features by function. 


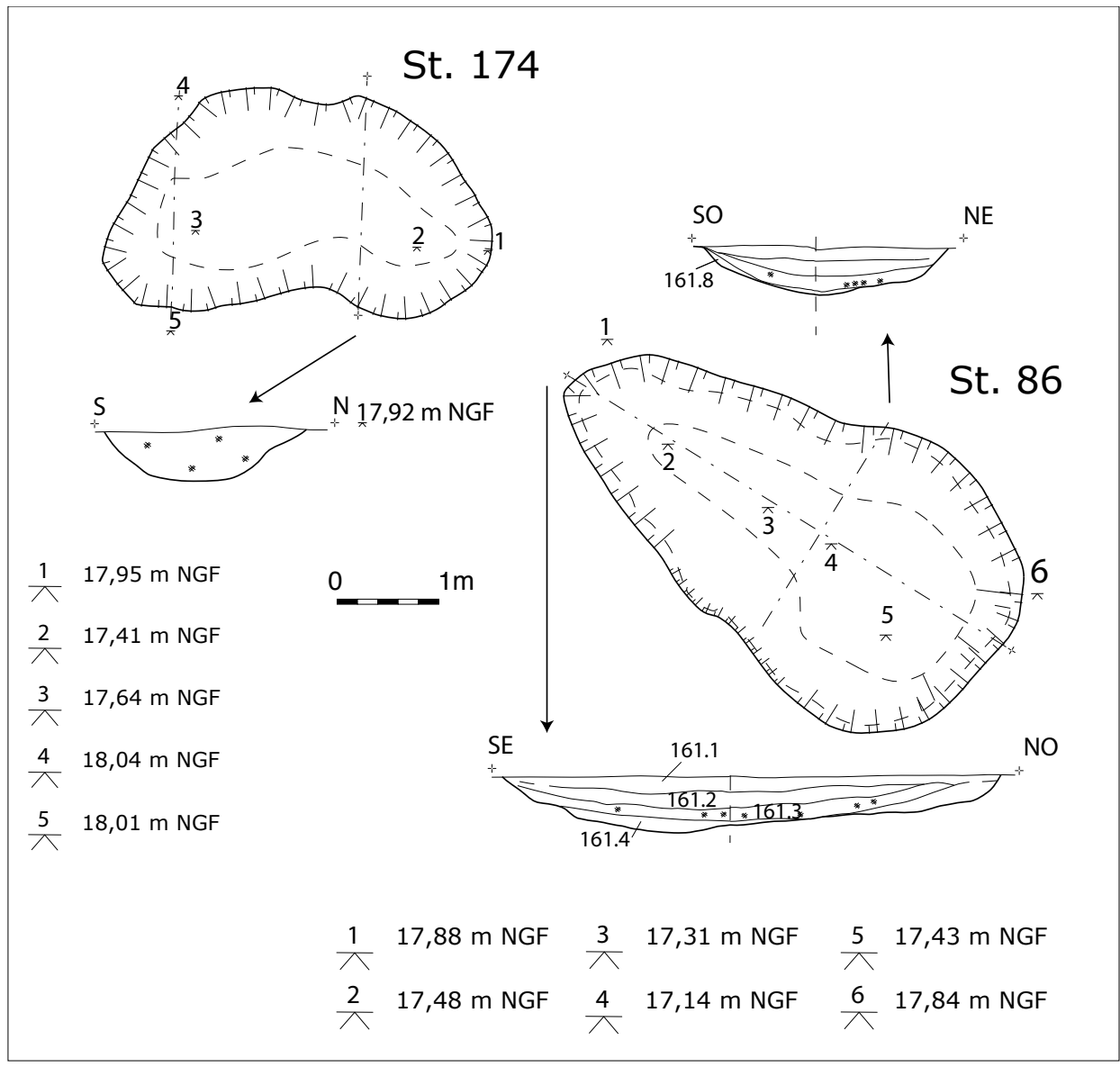

* Concentration de charbons

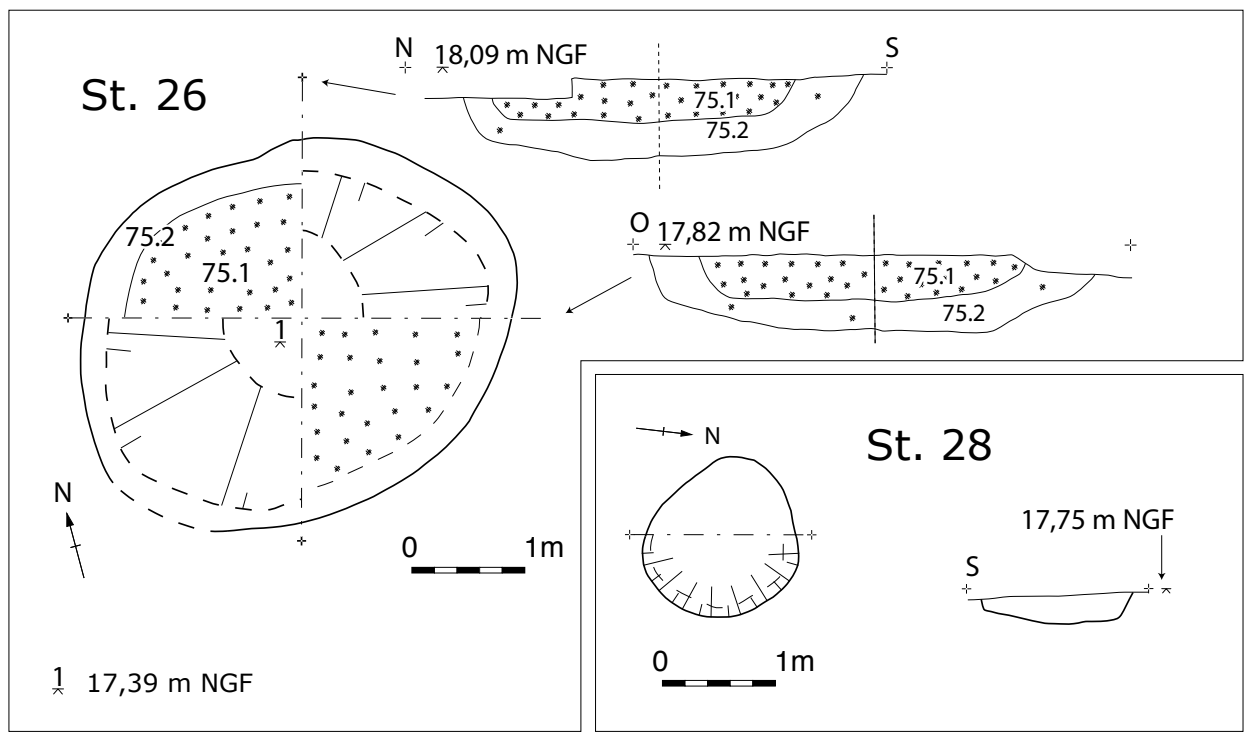

Figure 5 : Plan détaillé et coupes des structures attribuées au Néolithique ancien.

Figure 5: Detailed plan and sections dating to the Early Neolithic. présente comme une fosse de forme ovoïde, d'environ $3 \mathrm{~m}$ de diamètre, orientée est-ouest. Ses parois offrent un profil presque vertical et un fond plat. Deux comblements successifs ont été observés. Le remplissage initial de la structure est d'une trentaine de centimètres d'épaisseur, et constitué d'un limon argileux semi-compact et homogène, incluant quelques particules charbonneuses et rubéfiées. Reposant sur le limon, cet horizon présente un creusement plus important sur le pourtour de la fosse. Quelques éléments lithiques ont été recueillis dans cette couche. La couche supérieure se caractérise par un limon argileux comprenant une forte densité de charbons et de graines carbonisées, ce qui ne permet pas d'affirmer l'hypothèse d'une structure de stockage, tout au plus celle 


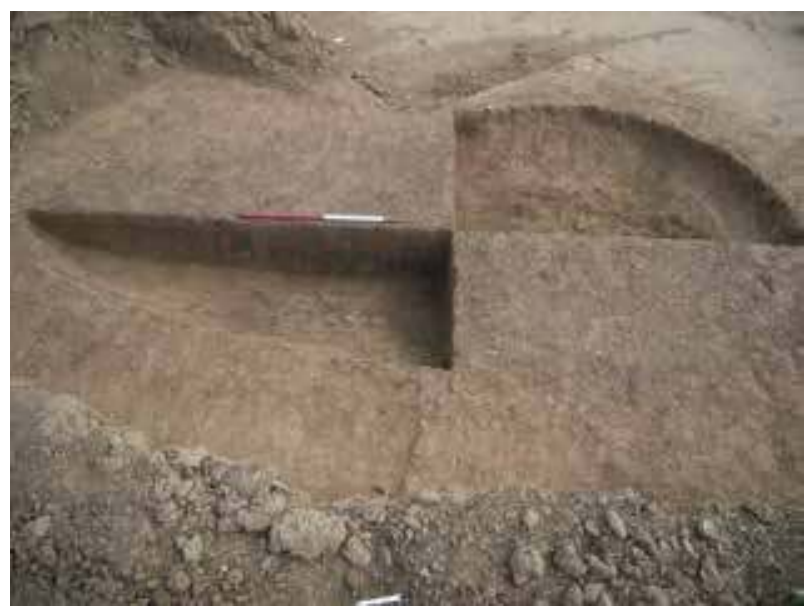

Figure 6 : Vue d'ensemble de la fosse 86.

Figure 6: Overview of pit 86.

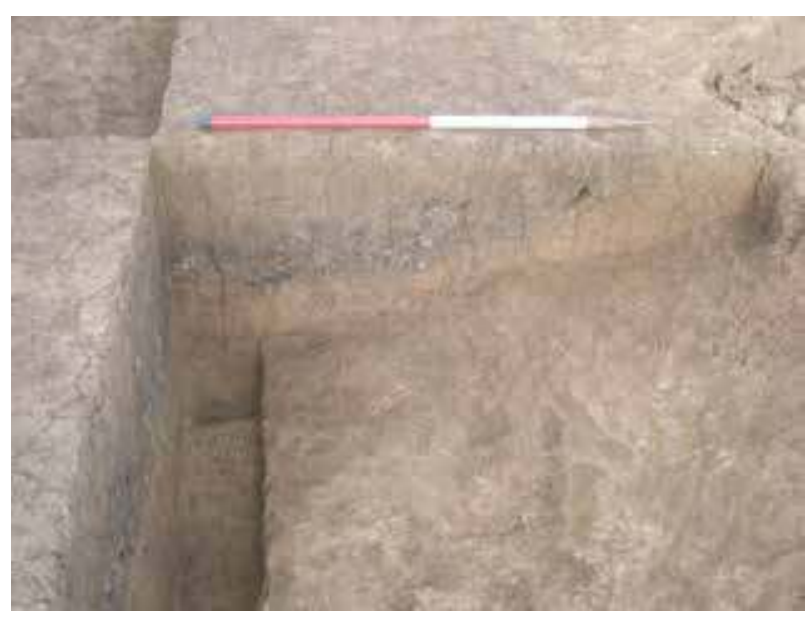

Figure 7 : Coupe de la fosse 86.

Figure 7: Section of pit 86.

d'une concentration de graines. Cette structure se signale par ailleurs par la présence d'un grand pic massif en grès qui reposait sur le fond de la structure.

Cette fosse a fait l'objet d'une datation AMS sur carporeste, dont le résultat est le suivant : Lyon-7800(GrA) : [5201 à 4847] av. J.-C. cal.

\section{- La fosse 28 (fig. 5 et g)}

La structure 28 n'est éloignée que d'une douzaine de mètres de la structure 26. L'étude du mobilier montre que cette petite fosse est susceptible de se rattacher à l'occupation du Néolithique ancien. Elle présente un plan subcirculaire orienté grossièrement est-ouest, de 1,28 m pour $0,26 \mathrm{~m}$ de profondeur. Le profil est abrupt, à parois verticales, et son fond est irrégulier et concave. Le remplissage limono-argileux, homogène et compact, de couleur brun-

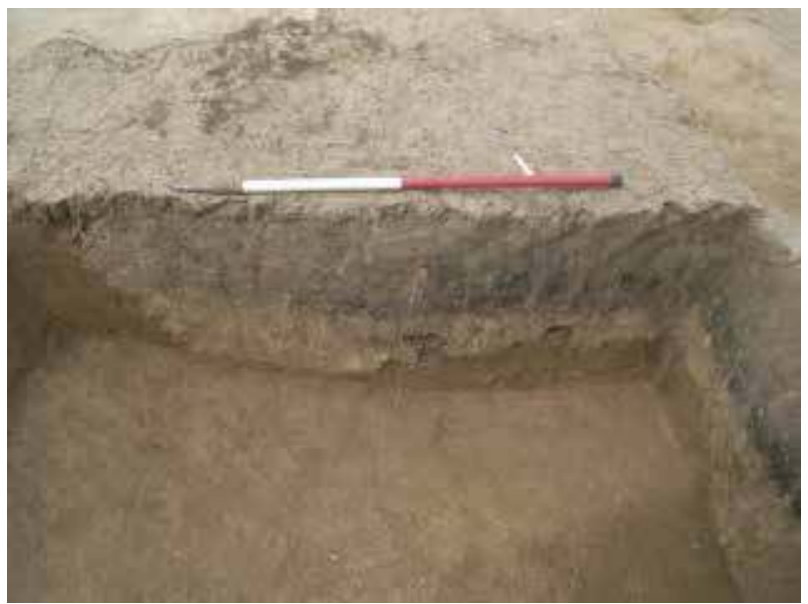

Figure 8 : Coupe de la st. 26.

Figure 8: Section of pit 26.

morphologie évoque une possible fonction de silo : aucun macroreste n'y a cependant été retrouvé. Le mobilier en présence, uniquement constitué d'éléments lithiques, renvoie à une production du Néolithique ancien (voir infra). Elle a livré 33 pièces, dont un grattoir sur lame. La totalité de l'industrie est laminaire et sur silex du Cinglais. Mais la pauvreté du mobilier ne permet pas d'être catégorique sur cette détermination. On ne dispose pas de datation par radiocarbone pour cette fosse.

\section{Le mobilier céramique du Néolithique ancien}

La céramique du Néolithique ancien du site de Démouville représente un petit corpus, dont le faible nombre de tessons est compensé par une certaine variété de décors ${ }^{1}$. Elle provient du comblement des grandes fosses 86 et 174 qui correspondent à une rangée de fosses latérales d'habitation relevant très probablement d'une même phase de fonctionnement. Le remplissage de la fosse 174 a permis de mettre au jour sept tessons $(23 \mathrm{~g})$ appartenant à deux récipients. Il s'agit de poteries fines à pâte sableuse ( 6 et $7 \mathrm{~mm}$ ) aux surfaces lissées. La faiblesse du mobilier céramique disponible ne permet pas de statuer sur la chronologie de cette structure. Cependant, sa proximité avec la fosse 86 laisse supposer qu'elle appartient au même horizon chronoculturel. Les comblements sommitaux de la fosse 86 ont fourni 139 tessons (261 g; tabl. 1). Ces derniers sont relativement bien conservés mais très fragmentés (2 g par tesson). Le nombre minimum d'individus identifiés est de neuf vases pour un nombre maximum de quatorze récipients. Cet ensemble est modeste, mais les éléments de forme et surtout les décors identifiés témoignent de son insertion chronologique.

1. Remerciements à Mike Ilett et Claude Constantin pour leur aide dans l'analyse de ce mobilier 


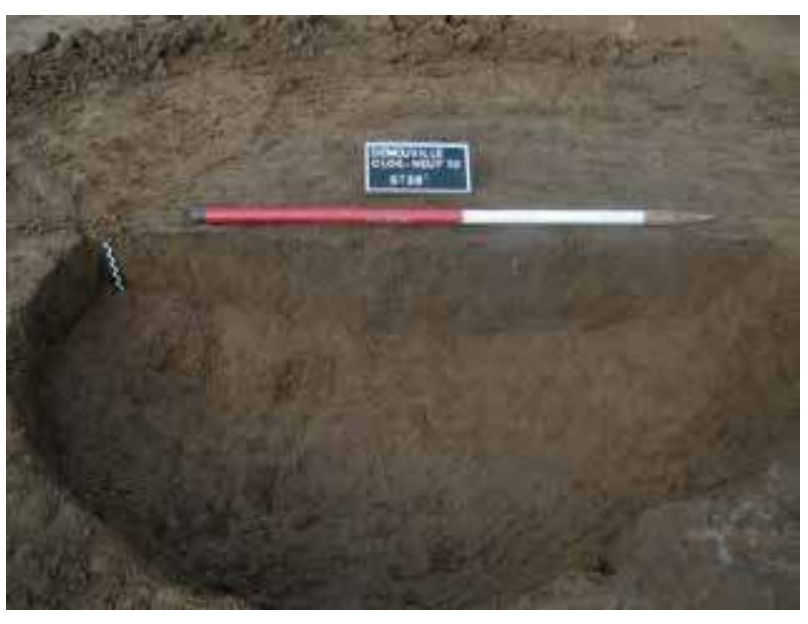

Figure 9: Vue et coupe de la st. 28.

Figure 9: View and section of the structure 28.

\section{- Les techniques de fabrication}

Lidentification des inclusions visibles dans les poteries a permis de caractériser au moins trois groupes macroscopiques de terre argileuse. Les céramiques vacuolaires (5) se distinguent par une forte porosité et ne renferment que de rares inclusions. Les matériaux employés pour la confection de ces récipients pourraient être des marnes bioclastiques disponibles dans la Plaine de Caen. Les céramiques limoneuses (5) renferment quant à elles assez peu d'inclusions, quelques quartz et de fines particules de micas. L'origine de ces matériaux n'est pas identifiée, il pourrait s'agir de limons argileux disponibles aux environs du site. Les céramiques à particules sableuses (4) se caractérisent par la présence de nombreux quartz arrondis ou anguleux, associés à des micas. Un individu comporte de nombreuses esquilles de silex, d'origine naturelle ou bien volontairement ajoutées comme dégraissant (fig. 10 et $11: 86.8$ ). L'ajout volontaire d'un dégraissant est visible sous la forme d'éléments arrondis et anguleux de couleur gris-beige à noir. Il s'agit très probablement de chamotte ajoutée dans la terre argileuse de quatre poteries.

Le montage au colombin est observé par quelques cassures en biseau et orientations préférentielles de celles-ci. Les surfaces des poteries sont bien lissées, seuls trois vases d'aspect grossier soulignent un faible investissement sur les surfaces. Une poterie révèle un tracé préparatoire pour la réalisation du décor (fig. 10 et $11: 86.7$ ). L'épaisseur des parois des vases varient de 4 à $10 \mathrm{~mm}$, avec une épaisseur moyenne de $6 \mathrm{~mm}$. Les surfaces des vases sont de couleur beige orangé à brun-noir, le cœur est principalement gris à noir. Un récipient conserve sur sa surface interne des restes de résidus de cuisson.

\section{- Les formes et les éléments de préhension-suspension}

Un bord à lèvre amincie est identifié. Il correspond probablement à un vase hémisphérique ouvert. La taille réduite du tesson ne permet pas d'estimer son diamètre à l'ouverture. Ses parois ont une épaisseur de $6 \mathrm{~mm}$ et ses surfaces sont lissées (fig. 10 et $11: 86.1$ ).

Deux poteries sont pourvues d'éléments de préhension et/ou suspension. Le premier est très érodé et n'est par conséquent pas identifiable. Le second est aussi fragmenté, mais il s'agit vraisemblablement d'un bouton perforé (fig. $10: 86.2$ ).

\section{- Les décors}

Dans cet ensemble, sept vases sont décorés par des impressions et des incisions (fig. 10 et 11). Ces techniques décoratives sont conjuguées dans trois cas.

L'impression est mise en œuvre par l'intermédiaire de peignes (3), de poinçons (2) et d'une spatule (1). Les peignes, employés de manière pivotante, mesurent de 3 à $5 \mathrm{~mm}$ de largeur. Il est difficile de discerner sur une poterie s'il s'agit d'un peigne à deux ou à trois dents puisque les impressions sont mal conservées (86.4). L'autre céramique est décorée d'impressions au peigne à trois dents parfois moins bien conservées (86.7). Un autre tesson très érodé révèle de possibles impressions au peigne à trois dents (86.3). Les poinçons ont, dans un cas, une extrémité arrondie de $1 \mathrm{~mm}$ (86.6) et, dans l'autre cas, une extrémité quadrangulaire de $3 \mathrm{~mm}$ (86.1). Une dernière poterie est décorée d'impressions à la spatule, voire au tranchant d'outil à front légèrement curviligne (86.8).

Les incisions sont fines (86.7) ou larges, de l'ordre du millimètre à profil en $U(86.3,86.6$ et 86.8$)$. Un récipient (86.5) est entaillé par de larges incisions, associées à des incisions plus fines pour former une grille.

La fragmentation des tessons ne permet de distinguer que les motifs décoratifs : les thèmes sont incomplets, tandis que les registres et la structure décorative sont le plus souvent hypothétiques.

Un décor du bord est identifié par un thème de ruban horizontal incisé, hachuré en échelle (86.7).

Les décors principaux les mieux représentés sont les thèmes de bandes verticales. Leurs motifs sont formés d'au moins une bande imprimée au peigne, d'une bande oblique imprimée au poinçon, de lignes incisées, de trois lignes incisées cernées par des impressions au poinçon et de lignes incisées délimitées par une bande d'impressions à la spatule, voire au tranchant d'outil ou même à l'ongle. Les autres décors sont formés par un thème rayonnant constitué de bandes d'impressions au peigne et par un thème couvrant composé d'une succession de fines incisions recoupées par des incisions plus larges. 


\begin{tabular}{|c|c|c|c|c|c|c|c|c|c|c|c|}
\hline US & Ind. & $\mathbf{N r}$ & $\begin{array}{l}\mathrm{PNr} \\
\text { g. }\end{array}$ & B & D & $\mathbf{P}$ & Aspect & $\begin{array}{l}\text { Ep. } \\
\text { mm }\end{array}$ & S Ext./Cœur/ S Int. & Pâte & Observation \\
\hline 1 & 86.3 & 2 & 8,4 & - & 1 & - & Bosselé & 7 & Beige orangé/Noir/Brun noir & Vacuolaire & Vac. $=$ bioclastes? \\
\hline 1 & 86.11 & 2 & 6,5 & - & - & - & Lisse & 5 & Noir/Noir/Noir & Sableuse & - \\
\hline 2 & 86.8 & 4 & 29,3 & - & 1 & - & Rugueux & 7 & Brun Noir/Noir/Noir & Sabl silex & 1 ind $4 \mathrm{~T} 4$ décors \\
\hline 2 & 86.5 & 1 & 1 & - & 1 & - & Lisse & 5 & Noir/Noir/Noir & Limoneuse & 1 ind $2 \mathrm{~T} 2$ décors \\
\hline 2 & 86.11 & 4 & 16,9 & - & - & - & Lisse & 6 & Noir/Noir/Noir & Sableuse & - \\
\hline 2 & 86.12 & 52 & 52,5 & - & - & - & Bosselé & 6 & Beige orangé/Gris/Gris & Vacuolaire & - \\
\hline 2 & 86.3 & 2 & 5,4 & - & - & - & Lisse & 8 & Beige orangé/Noir/Brun noir & Vacuolaire & Vac.= bioclastes? \\
\hline 2 & 86.1 & 4 & 7,1 & - & - & - & Lisse & 10 & $\begin{array}{c}\text { Beige orangé/Gris/Beige } \\
\text { orangé }\end{array}$ & Vacuolaire & Incl arr gris/beige $=$ cham? \\
\hline 2 & - & 9 & 6,3 & - & - & - & - & - & - & - & Esquilles \\
\hline 2 & 86.13 & 6 & 8,5 & - & - & 1 & Lisse & 7 & Brun noir/Noir/Brun noir & Vacuolaire & Préhension très abimé \\
\hline 2 & 86.14 & 2 & 3,2 & - & - & - & Lisse & 7 & Beige/Gris/Beige & Limoneuse & Incl gris/bleu noir=cham! \\
\hline 3 & 86.1 & 2 & 3,9 & 1 & 1 & - & Lisse & 6 & Beige orangé/Gris/Gris & Vacuolaire & Incl arr gris/beige $=$ cham? \\
\hline 3 & 86.2 & 1 & 3,8 & - & - & 1 & Lisse & 5 & Gris brun/Gris/Gris brun & Limoneuse & - \\
\hline 3 & 86.9 & 3 & 20,3 & - & - & - & Lisse & 6 & Beige orangé/Gris/Brun & Sableuse & Incl ang noires=cham? \\
\hline 3 & 86.7 & 5 & 22,8 & - & 1 & - & Lisse & 4 & Brun Noir/Gris/Brun & Limoneuse & $\begin{array}{l}\text { Incl vég? } 1 \text { ind } 5 \mathrm{~T} \\
5 \text { décors }\end{array}$ \\
\hline 3 & 86.5 & 1 & 4,7 & - & 1 & - & Lisse & 8 & Noir/Noir/Noir & Limoneuse & 1 ind $2 \mathrm{~T} 2$ décors \\
\hline 3 & 86.6 & 5 & 12 & - & 1 & - & Lisse & 5 & Brun/Brun noir/Brun noir & Sableuse & $\begin{array}{c}\text { Incl orga } 1 \text { ind } 3 \mathrm{~T} \\
\text { 3décors }\end{array}$ \\
\hline 3 & 86.4 & 7 & 4,1 & - & 1 & - & Lisse & 5 & Brun orangé/Orangé/Orangé & Limoneuse & Incl arr gris/blanc=cham? \\
\hline 3 & 86.3 & 3 & 2,7 & - & - & - & Bosselé & 7 & Beige orangé/Noir/Brun noir & Vacuolaire & Vac. $=$ bioclastes? \\
\hline 3 & 86.9 & 1 & 4,1 & - & - & - & Lisse & 6 & Beige orangé/Gris/Brun & Limoneuse & Résidu cuisson int \\
\hline 3 & 86.10 & 3 & 11,7 & - & - & - & Lisse & 14 & Beige/Gris/Rouge orangé & Vacuolaire & - \\
\hline 3 & 86.11 & 17 & 24,7 & - & - & - & Lisse & 5 & Noir/Noir/Noir & Sableuse & - \\
\hline 3 & - & 3 & 1,7 & - & - & - & - & - & - & - & Esquilles \\
\hline
\end{tabular}

Tableau 1: Données quantitatives et pondérales du mobilier céramique de la fosse 86.

Table 1: Quantitative and weight data of pottery from pit 86.

\section{- Le vase 86.7 (fig. 10 et 11)}

Plusieurs tessons permettent de proposer une reconstitution d'une grande partie de l'organisation des décors de ce vase. Son thème principal en chevron est constitué d'un motif en ruban incisé à remplissage de lignes transversales incisées, légèrement obliques. Ces chevrons s'accrochent à une bande incisée en échelle qui longe le bord. Les autres décors secondaires sont constitués de bandes d'impressions au peigne formant de possibles thèmes rayonnants et par des bandes verticales imprimées au peigne, disposées de part et d'autre du probable thème en chevron.

\section{- De possibles tessons Limbourg}

Claude Constantin a observé chacun des tessons à la binoculaire et a rapproché ses observations de celles de
Mike Ilett. Malgré la fragmentation de la série, il est possible de reconnaître quelques éléments Limbourg comme le décor de grille incisée dans la pâte fraîche (86.5) et celui de lignes verticales incisées parallèles, margées d'impressions à la spatule (86.8). Il en est peut-être de même pour le 86.3 dont le décor de bande imprimée margée d'incisions est trop effacé pour être affirmatif.

\section{- Attribution chronoculturelle et comparaisons}

Le mobilier céramique de Démouville se rapporte incontestablement aux toutes premières occupations néolithiques en Normandie. Les décors ont de fortes affinités avec ceux de la fin du Rubané final du bassin de la Seine, anciennement étape finale du RRBP (Constantin et Ilett, 1997; Constantin, 2013; Ilett et Meunier, 2013). En effet, dans cette étape, les impressions sont principalement pivo- 


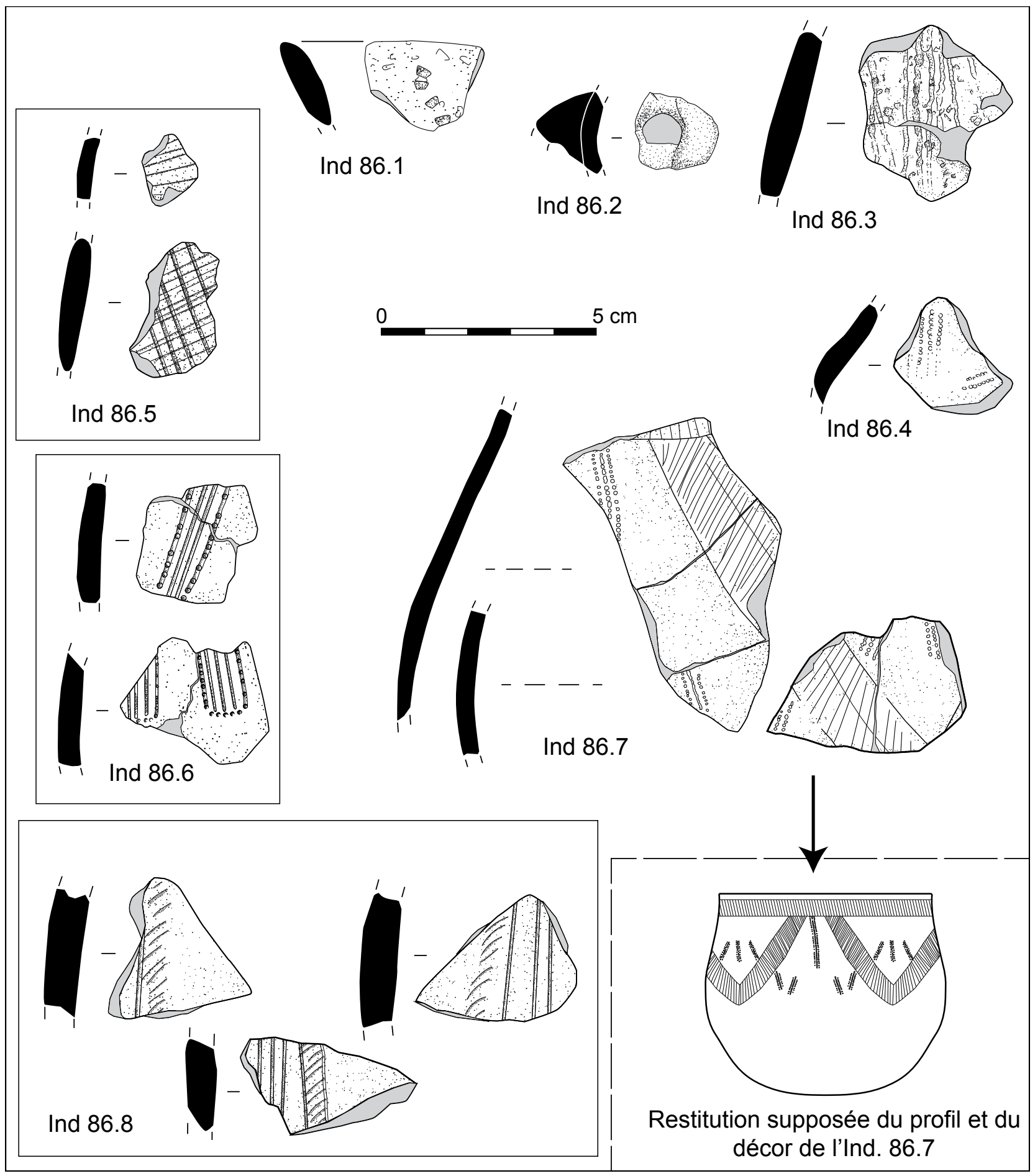

Figure 10 : Démouville « ZAC du Clos Neuf», mobilier céramique de la st. 86 et interprétation de la structure décorative.

Figure 10: Démouville "ZAC du Clos Neuf", pottery from structure 86 and interpretation of the ornemental motifs.

tantes avec l'emploi de peignes de deux à trois dents. Les décors sur le bord sont régulièrement constitués de rubans hachurés horizontaux souvent associés à un décor principal du même type. Le décor principal en chevron est également bien identifié à cette étape, souvent formé de rubans incisés remplis de lignes transversales incisées. Les décors secondaires sont principalement réalisés par impression au peigne. Enfin, la céramique du Limbourg est présente dans un peu moins de la moitié des sites de cette période.

Les motifs en ruban incisé à remplissage de lignes incisées présents à Démouville semblent être typiques de la fin du Rubané final du bassin de la Seine (fig. 10 : 86.7). Ils sont bien représentés sur les sites de Colombelles "Le Lazzaro ", de Chaumont " les Grahuches » ou 


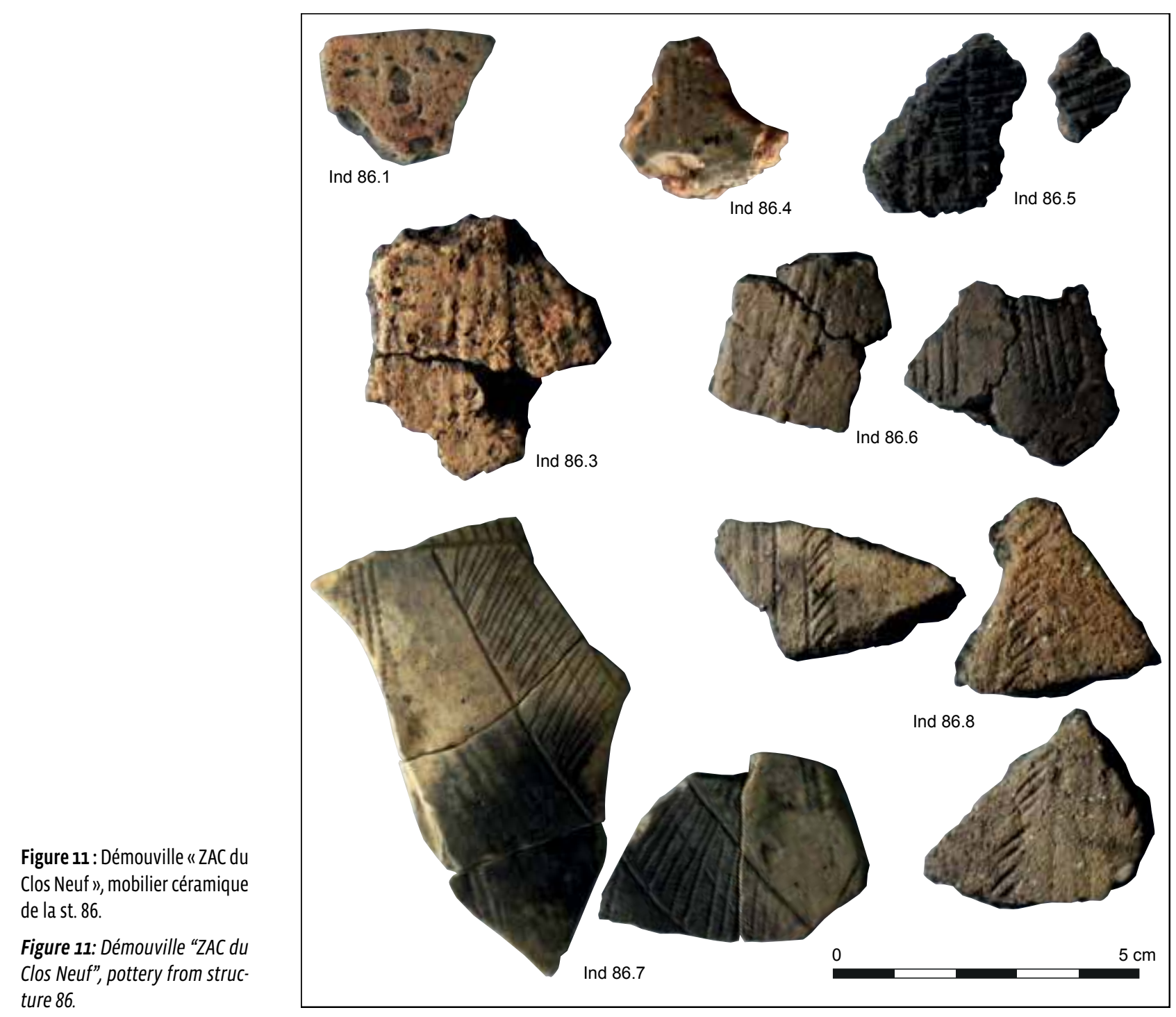

encore de Balloy " les Réaudins" (Billard et al., 2014²; Meunier, 20123). Sur le site de Démouville, deux poteries (fig. $10: 86.5$ et 86.8 ) et une possible troisième (fig. $10: 86.3$ ) ont des décors apparentés à la céramique du Limbourg. Les fouilles archéologiques récentes démontrent la présence de cette céramique dans la région, mais elle était jusqu'à présent extrêmement rare. En effet, seuls deux petits tessons évoquant la céramique Limbourg sont reconnus sur le site de Colombelles « Le Lazzaro " proche de celui de Démouville (Billard et al., 2014 ${ }^{4}$ ). Le site de Moult «Le Relais de la Poste " (14) en a aussi livré récemment quelques tessons (Ghesquière et al., 2018)

Les décors en thème couvrant de larges incisions entrecroisées, de larges incisons verticales cernées de courtes

2. Fig. 74 st. $16-1$ n $^{\circ} 3$ et 4 , fig. 79 st. 55 n 6 , fig. 89 st. $464 n^{\text {os }} 1$ et 2

3. Pl. 5 st. 7 n $^{\circ} 7$, pl. 7 st. 130 n $^{\circ}$ 5, pl. 18 st. 1 no $^{\circ} 10$. incisions sont fréquemment employés dans les céramiques du Limbourg comme sur les sites d'Aubechies « CoronMaton ", de Cuiry-lès-Chaudardes "Les Fontinettes ", de Juvigny "Les Grands Traquiers » ou encore de PontSainte-Maxence « Le Joncquoire II » (Constantin, 1985; Meunier, 2013a; Alix et al., 1997). Le motif de lignes incisées bordées d'impressions à la spatule (fig. 10 : 86.8) trouve des équivalents sur les sites du RRBP de Barbey «Le Buisson rond » et de Passy « Les Graviers » (Meunier, $2012^{5}$ ). Ces différentes comparaisons permettent de placer la fosse 86 du site de Démouville à la fin du Rubané final du Bassin de la Seine.

Malgré la modestie du corpus de Démouville, des différences avec la céramique de Colombelles sont toutefois perceptibles : la céramique de type Limbourg semble mieux représentée et le récipient 86.7 fait appel, selon une grammaire rubanée, à des décors secondaires qui sont

5. Pl. 9 st. 18 n $^{\circ}$ 2, pl. 65 st. 3 n $^{\circ} 10$. 
totalement absents à Colombelles. D'ailleurs, ces décors secondaires disparaîtront du corpus régional B-VSG.

\section{Le mobilier lithique du Néolithique ancien}

Le mobilier en silex de la phase rubanée concerne un bon millier d'objets et provient des fosses 28,86 et 174 (tabl. 2). La majorité de l'effectif est concentrée dans la structure 86 (894 objets sur 1042 ). Le matériel comprend une forte proportion de débris (75\%), parmi lesquels de nombreuses esquilles et débris thermiques, dont la surreprésentation est liée à la finesse de la fouille et au tamisage des déblais. On compte 158 déchets de taille, technologiquement significatifs (15\%), et 104 outils (10\%).

Les matières premières reconnues dans la série sont régionales à $96 \%$ : il s'agit essentiellement de différentes variantes de silex jurassiques de la Plaine de Caen, proches en qualité du silex du Cinglais. Ces matériaux sont employés dans la région durant tout le Néolithique en raison de leur grande qualité, et à plus forte raison au Néolithique ancien où ils font l'objet d'une extraction minière liée à une production de lames standardisées qui diffuse sur de longues distances. Les autres matériaux reconnus dans la série sont très rares : il s'agit principalement de ressources de la vallée de la Seine et du Pays de Caux. Ces ressources ne sont pas représentées en quantité suffisante pour supposer un apport régulier et planifié, mais plutôt un apport anecdotique.

Les déchets de taille témoignent de la coexistence de deux chaînes opératoires de débitage dans la série : lames et éclats. Aucun déchet technique issu d'une chaîne opératoire de façonnage n'a été observé. Plusieurs éclats de dégrossissage épais et éclats de préparation de nucléus témoignent sans doute d'un apport de rognons partiellement préparés sur le site, puis entièrement débités sur place. Les déchets de débitage laminaire témoignent d'une méthode très caractéristique du Néolithique ancien, dans la région comme dans le reste du Bassin parisien (Bostyn et al., 2019). Ils témoignent de la mise en place d'une crête frontale (une pièce à crête) produisant des déchets caractéristiques (6 éclats de préparation de crête). De nombreux cassons de lames ou supports laminaires témoignent des intentions des tailleurs : 3 lames entières brutes, 25 fragments de lames et 30 lames transformées en outils. Les produits sont conformes aux productions caractérisées dans la région pour le reste du Néolithique ancien (Charraud, 2015) : il s'agit de produits très réguliers, aux plans de frappe lisses, détachés exclusivement par percussion indirecte. Les lames sont de petit module avec une longueur recherchée estimée à 8 ou $9 \mathrm{~cm}$ en moyenne. Les éclats d'entretien (12 tablettes d'avivage, 25 éclats d'entretien de carène) témoignent de la grande régularité du débitage et de la bonne maîtrise technique

\begin{tabular}{|c|c|c|c|c|}
\hline Déchets de taille & $\begin{array}{l}\text { St. } \\
86\end{array}$ & $\begin{array}{c}\text { St. } \\
174\end{array}$ & $\begin{array}{l}\text { St. } \\
28\end{array}$ & Total \\
\hline Éclat de dégrossissage & 20 & 0 & 1 & 21 \\
\hline Éclat prépa crête & 4 & 2 & 0 & 6 \\
\hline Pièce à crête & 0 & 0 & 1 & 1 \\
\hline Tablette d'avivage & 12 & 0 & 0 & 12 \\
\hline Éclat entretien carène & 15 & 5 & 5 & 25 \\
\hline Lame entière PDD & 3 & 0 & 0 & 3 \\
\hline Lame fragment prox. PI & 13 & 4 & 2 & 19 \\
\hline Lame fragment més. & 8 & 2 & 1 & 11 \\
\hline Lame fragment dist. & 6 & 0 & 0 & 6 \\
\hline Lamelle ou fragment & 7 & 0 & 1 & 8 \\
\hline Éclat allongé brut & 12 & 1 & 1 & 14 \\
\hline Éclat court brut & 15 & 11 & 3 & 29 \\
\hline NE multidirectionnel & 2 & 1 & 0 & 3 \\
\hline Total débitage & 117 & 26 & 15 & 158 \\
\hline \multicolumn{5}{|l|}{ Débris } \\
\hline Esquille & 625 & 69 & 10 & 704 \\
\hline Casson d'éclat & 61 & 7 & 5 & 73 \\
\hline Casson de nucléus & 3 & 0 & 0 & 3 \\
\hline Total débris & 689 & 76 & 15 & 780 \\
\hline \multicolumn{5}{|l|}{ Outillage } \\
\hline Grattoir sur éclat & 5 & 3 & 0 & 8 \\
\hline Grattoir sur lame & 4 & 3 & 1 & 8 \\
\hline Burin sur éclat & 2 & 0 & 0 & 2 \\
\hline Burin sur lame & 2 & 0 & 0 & 2 \\
\hline Perçoir (mèche) & 1 & 0 & 0 & 1 \\
\hline Éclat retouché/écaillé & 32 & 3 & 2 & 37 \\
\hline Éclat denticulé & 1 & 0 & 0 & 1 \\
\hline Lame retouchée/écaillée & 9 & 0 & 0 & 9 \\
\hline Lame tronquée & 2 & 0 & 0 & 2 \\
\hline Armature tranchante & 4 & 0 & 0 & 4 \\
\hline Armature danubienne & 3 & 3 & 0 & 6 \\
\hline Pièce esquillée & 13 & 1 & 0 & 14 \\
\hline Pièce à encoche & 2 & 0 & 0 & 2 \\
\hline Pièce bouchardée & 4 & 0 & 0 & 4 \\
\hline Frag. pièce bouchardée & 5 & 0 & 0 & 5 \\
\hline Total outillage & 89 & 13 & 3 & 105 \\
\hline Total série & 895 & 115 & 33 & 1043 \\
\hline
\end{tabular}

Tableau 2 : Décompte de l'industrie lithique des structures du Néolithique ancien.

Table 2: Lithic counts from the Early Neolithic features.

des tailleurs. La quantité des déchets de taille suffit à expliquer les lames présentes sur le site, mais ne permet pas de supposer une production excédentaire. 
Le débitage d'éclats est représenté par quelques éclats bruts courts (29) ou allongés (14) et trois nucléus multidirectionnels. Il ne présente pas de caractères particuliers. Il est entièrement réalisé par percussion directe dure selon un schéma opératoire simple, multidirectionnel et non standardisé. Il s'agit clairement, de manière classique dans les assemblages du Néolithique ancien, d'une production simple qui complète les besoins d'un outillage plus investi techniquement. La part des éclats produits spécifiquement pour les besoins de l'outillage est difficile à évaluer, car de nombreux supports ont été récupérés parmi les déchets de débitage laminaire.

La série comprend 104 outils, dont 88 pour la seule fosse 86. L'outillage oppose de manière classique un panel d'outils sur lames et un autre sur éclats ou débris. Les outils sur lame sont des grattoirs (8), des burins (2), des lames écaillées/retouchées (9) ou tronquées (2) (fig. 12). S'y ajoutent 10 armatures de flèches, dont 4 tranchantes et 6 perçantes asymétriques (fig. 13). L'outillage sur éclat et masse centrale concerne principalement des outils expédients typologiquement non normés : on trouve notamment 37 éclats écaillés ou retouchés (fig. 14). La quasi-absence des denticulés est remarquable. Les pièces esquillées sont en revanche très bien représentées avec 14 exemplaires (fig. 14).

\section{Le pic massif de la structure 26}

Un pic massif en grès a été découvert dans la structure 26. Il mesure $33 \mathrm{~cm}$ de long, $9 \mathrm{~cm}$ de large et pèse $2,6 \mathrm{~kg}$ (fig. 15). La matière première est un grès-quartzite d'âge primaire disponible dans un rayon de $20 \mathrm{~km}$ autour du site (Rioult et al., 1989a et b). Il présente globalement des sections prismatiques à quadrangulaires. La mise en forme de l'outil est réalisée par percussion directe au moyen de grands enlèvements, à partir d'un bloc présentant des surfaces naturelles diaclasées. Ce type d'objet est reconnu à plusieurs phases du Néolithique comme un outil de creusement. Ils existent en contexte de carrières ou de mines
Figure 12 : Démouville « Le Clos Neuf » : mobilier lithique du Néolithique ancien. Outils sur lame.

Figure 12: Démouville "ZAC du Clos Neuf" : Early Neolithic lithics. Tools on blade.

1 : burin dièdre ; $2,3,5$ à 7 : grattoirs sur lames; 4 : burin sur lame ; 8 à 11 : lames écaillées/retouchées.

1: "burin dièdre"; 2, 3, 5 à 7: scrappers on blades;
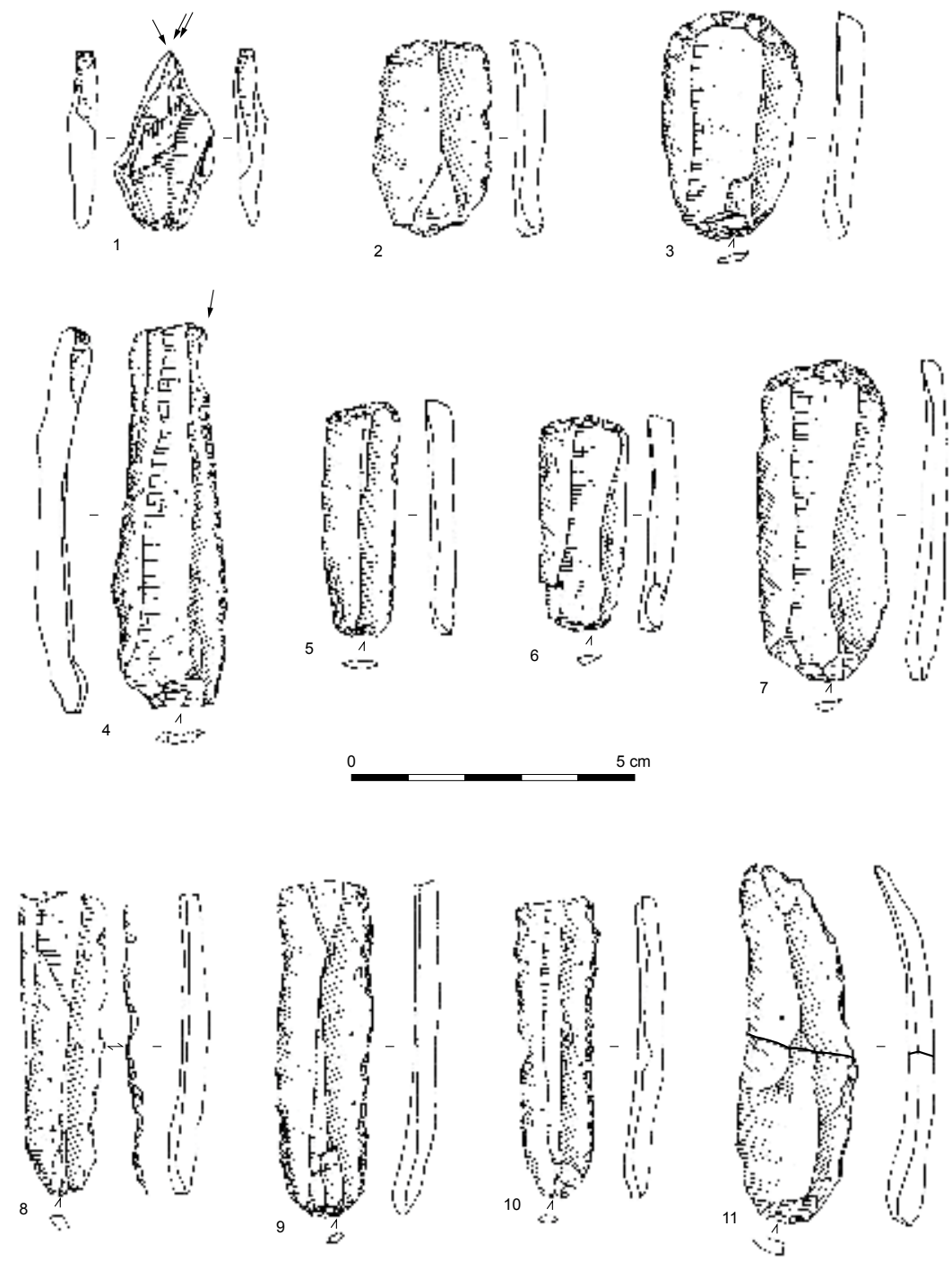
(Plussulien : Le Roux, 1999; Bretteville-le-Rabet : Desloges, 1986); mais également dans d'autres contextes où le creusement de substrats rocheux s'impose. Dans la région, on en trouve dans les sépultures type Passy à Fleury-sur-Orne (Ghesquière, 2019) ainsi qu’à Saint-Sylvain (Fromont et al., 2013), mais également en contexte domestique dans l'enceinte de Saint-Martin-de-Fontenay (Ghesquière et Marcigny, 2014). L'exemplaire de Démouville présente des émoussés très réguliers et très importants, postérieurs au détachement des éclats de mise en forme. Il ne s'agit donc pas d'émoussés naturels (érosion), mais bien d'émoussés d'origine fonctionnelle, difficiles à interpréter en l'état actuel. Cependant, d'importantes surfaces de la pièce sont restées brutes (surfaces diaclasées).
Initialement définis comme industries " montmorenciennes " en Île-de-France par L. Franchet et L. Giraux (1923), réévaluées par J. Tarrête (1977) et plus récemment par S. Griselin (2020), ces outils apparaissent aujourd'hui réservés à ces contextes fonctionnels très particuliers (creusement). Leur présence liée à leur fonction semble s'affranchir des limites chronoculturelles, bien qu'ils soient plus fréquents en contextes du premier Mésolithique et du Néolithique moyen.

\section{Les deux blocs d'hématite}

La fosse 86 a livré deux blocs d'hématite : le premier est un bloc oblong de $204 \mathrm{~g}$ présentant des surfaces polies au niveau de ses arêtes, le second est un débris de très
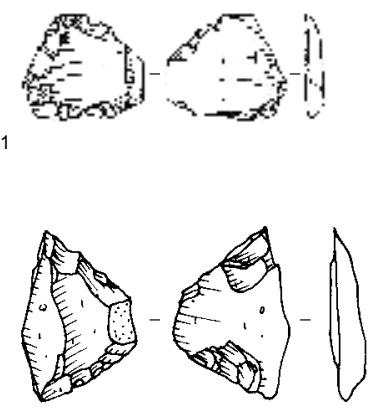

3
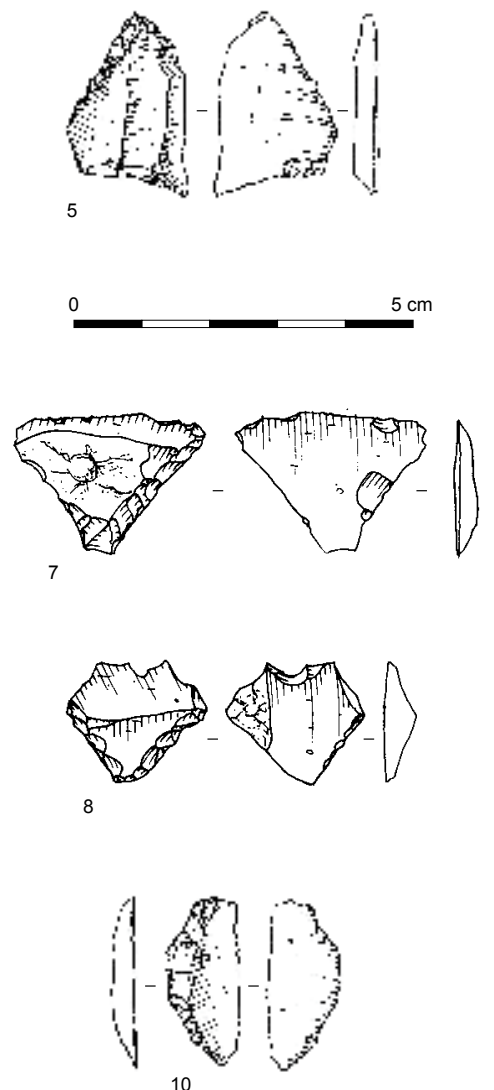
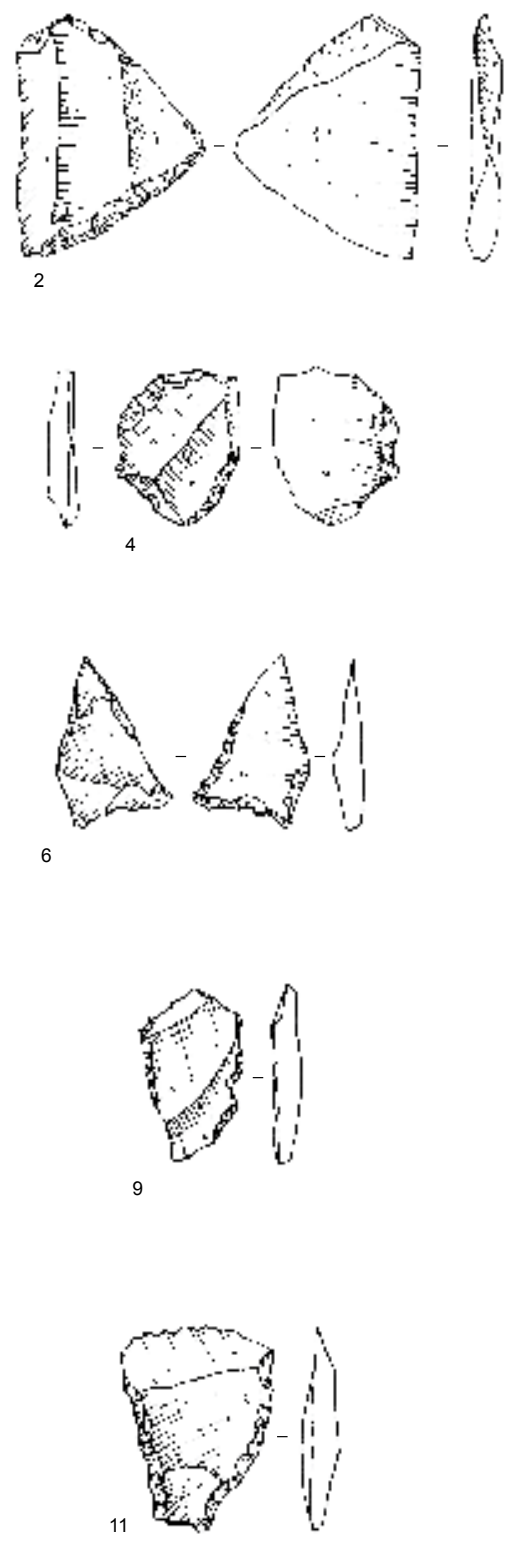

Figure 13 : Démouville « Le Clos Neuf » : mobilier lithique du Néolithique ancien. Armatures de flèches.

Figure 13: Démouville "ZAC du Clos Neuf": Early Neolithic lithics. Arrowheads. 
petites dimensions (fig. 16). Ce dernier objet a donné lieu à des analyses dans le cadre du PCR hématite mené avec nos collègues belges et qui s'est achevé par la table-ronde de Jambes (Belgique) [Billard et al., 2016a et 2016b; Goemaere et al., 2016a et 2016b]. Dans le cadre de ce travail, des analyses physico-chimiques ont été réalisées sur onze échantillons normands, dont celui de Démouville (Goemaere et al., 2016). On se référera à cette publication pour le protocole analytique et le détail des résultats obtenus. Ces échantillons ont été comparés à ceux des gisements géologiques disponibles en Normandie, en Belgique et dans l'Eiffel allemand.

Les principaux dépôts d'hématite normande datent de l'Ordovicien : ils sont issus de la remobilisation du fer issu de l'altération des terres émergées voisines (ancienne cor- dillère de Coutances) sous forme d'oolithes ferrugineuses riches en hématite, chlorite et sidérite. Ces formations ont été largement plissées à la fin de l'ère primaire. Les affleurements sont donc accessibles, au sud de la Plaine de Caen, sur les flancs sud et nord des synclinaux, dont les trois principaux sont (du nord au sud) le synclinal de May, le synclinal d'Urville, le synclinal de la zone bocaine. Vers l'est et le sud, c'est-à-dire en position distale par rapport à la péninsule, on trouve un minerai moins riche en hématite et principalement composé de sidérite et de chlorite, qui n'a pas de qualité colorante. Les gisements ont une épaisseur importante allant de 3 à $6 \mathrm{~m}$.

L'échantillon d'hématite oolithique de Démouville est tout à fait compatible avec ceux des sources géologiques échantillonnées régionalement à May-sur-Orne, Saint-
Figure 14 : Démouville «Le Clos Neuf »: mobilier lithique du Néolithique ancien. Outils sur éclats.

Figure 14: Démouville "ZAC du Clos Neuf”: Early Neolithic lithic industry. Tools on flakes.

1 : grattoir ; 2,5 et 6 : pièces esquillées ; 3 et 4 : éclats retouchés

1: scrapper; 2, 5 et 6: "pièces esquillées";
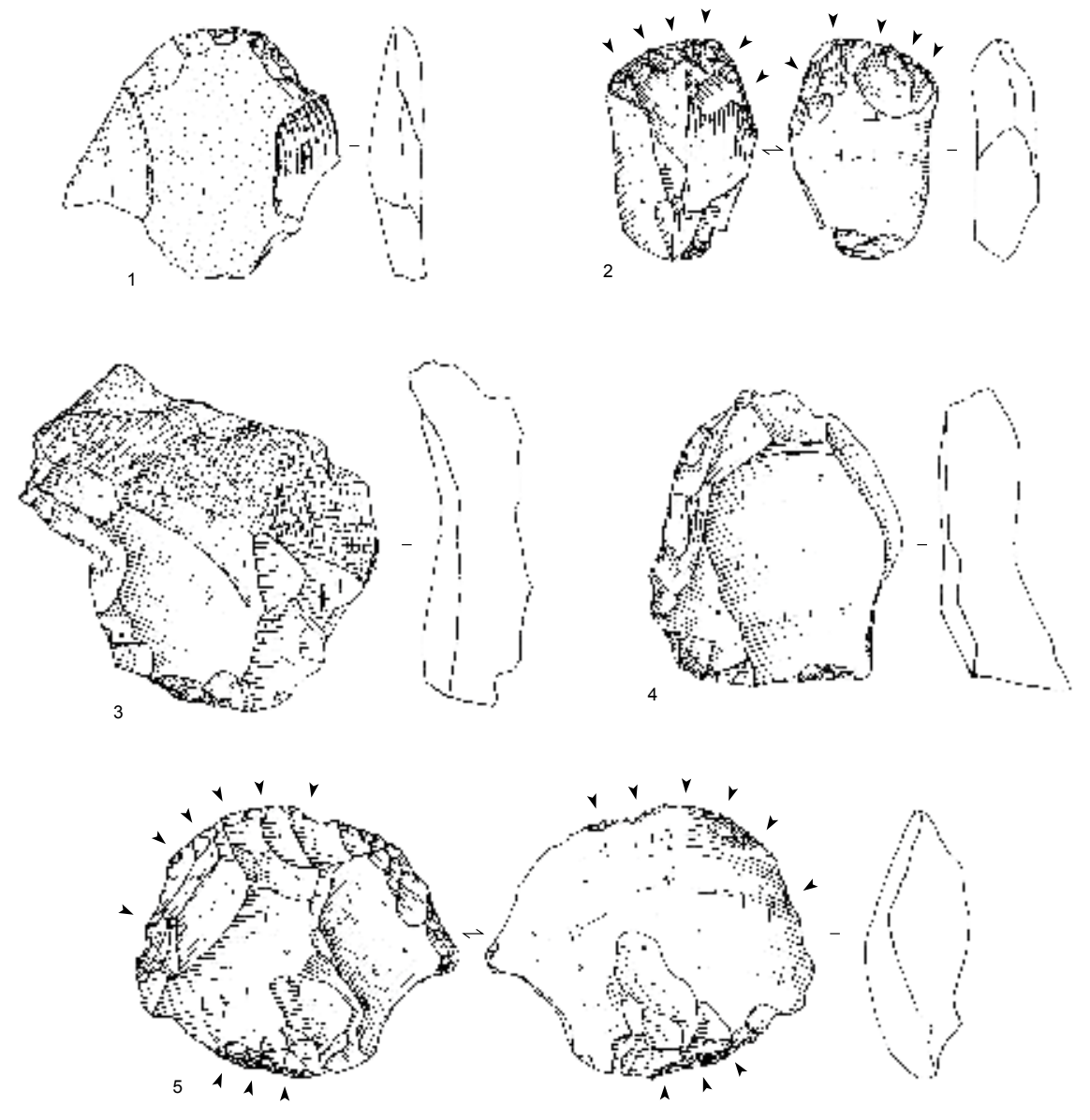

4
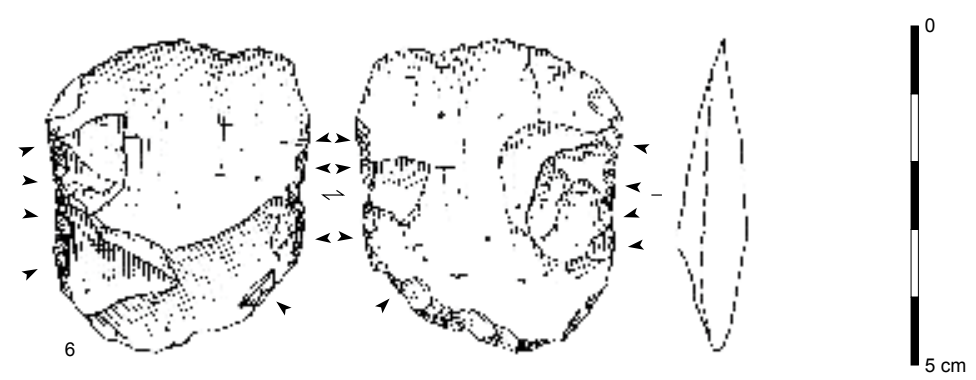

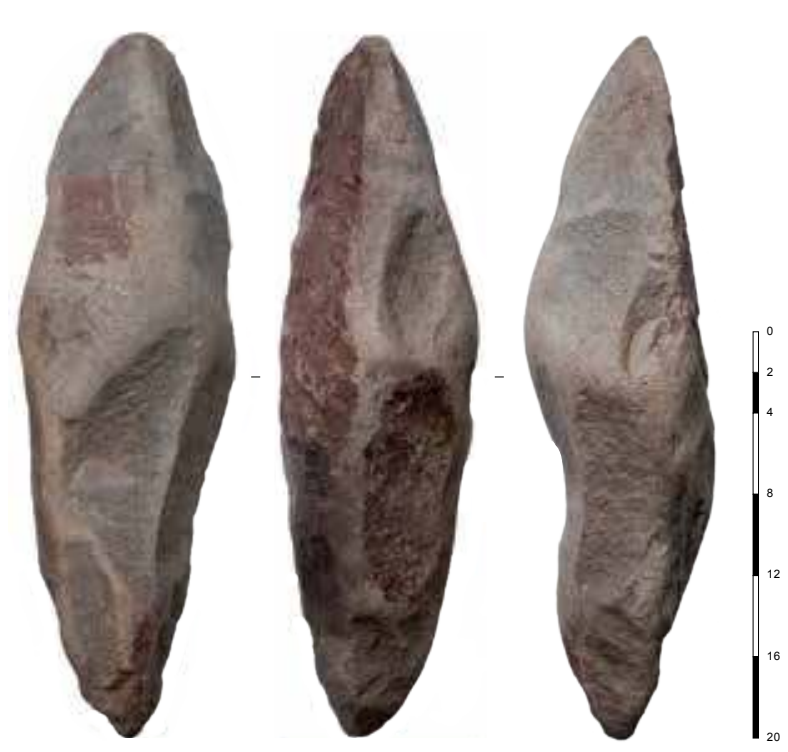

Figure 15 : Démouville « Le Clos Neuf » : mobilier lithique du Néolithique ancien. Pic massif en grès provenant de la st. 26.

Figure 15: Démouville "ZAC du Clos Neuf": Early Neolithic lithics. Sandstone flint pick from st. 26.

Rémy et Urville (14). On ne retrouve donc aucun apport d'hématites dévoniennes belges (Famennien inférieur, Frasnien...) ni de Lorraine ni de l'Eiffel.

Comme les objets de Colombelles, les deux objets de Démouville proviennent de couches géologiques peu altérées indicatives d'une probable extraction par creusement et non d'un simple ramassage de surface. Ils suggèrent que l'activité minière, bien attestée maintenant pour l'extraction de silex (Desloges et al., 2010; Charraud, 2015), concerne également l'exploitation d'autres matériaux tels que l'hématite.

La mise en évidence d'une activité de production d'hématite oolithique en Normandie est très récente, avec notamment la fouille du site de Colombelles. La découverte d'hématite travaillée sur les sites de BiévilleBeuville "Le Vivier " (Mésolithique final) et Démouville semble indiquer un contexte d'utilisation " ancien " et trouvant son aboutissement lors de la néolithisation, avec de possibles transferts culturels entre populations autochtones et colons néolithiques. Les datations obtenues pour Colombelles et Démouville (à la charnière des $\mathrm{VI}^{\mathrm{e}}$ et $\mathrm{V}^{\mathrm{e}}$ millénaires) autorisent aujourd'hui des comparaisons avec la sphère rubanée classique.

La série normande comprend peu de vestiges en contexte archéologique : Colombelles avec un total de 148 restes, dont 20 abrasés, les deux blocs de Démouville, une dizaine d'éléments à Biéville-Beuville. Le reste du corpus est principalement constitué de blocs provenant de ramassages de surface.

La présence de nouveaux blocs d'hématite à Démouville confirme l'importance de cette activité d'extraction dans ce premier Néolithique de la Plaine de Caen. Avec la présence d'un gros bloc abrasé que l'on peut qualifier de portatif, elle montre toutefois une différence de dimension avec le site de Colombelles, qui n'a livré que des petits fragments et où l'on entrevoit une forte activité de broyage. Ce type d'objets portatifs n'a pour l'instant été observé que sur des objets issus de ramassages de surface et du site de Biéville-Beuville attribué à l'extrême fin du Mésolithique (Artur et Billard, 2008), et dont le caractère primaire et homogène est loin d'être bien établi.

\section{Les structures du Néolithique moyen \\ (st. 11, 24, 34, 3639 et 67) : (fig. 17)}

\section{- La structure 11}

La structure 11 se présente comme une fosse de plan plus ou moins circulaire, à bords légèrement convexes et fond concave. Son diamètre atteint $1,10 \mathrm{~m}$ pour une profondeur de $0,45 \mathrm{~m}$. Le remplissage supérieur scellant la structure, fortement charbonneux, évoque un rejet de foyer. Aucun macroreste n'a été perçu dans la matrice limono-argileuse. Cependant, son profil l'apparente à une fosse-silo, ayant pu fonctionner comme dépotoir lors de son comblement. Elle a fait l'objet d'une datation par le radiocarbone qui la situe au début du Chasséen : 5305 \pm 30 BP, soit [4235-4045] av. J.-C. cal.

\section{- La structure 24 (fig. 18)}

Situé dans la partie la plus septentrionale de l'emprise, le silo 24 se caractérise par une forme en plan circulaire de $1,50 \mathrm{~m}$ de diamètre, un profil en cuvette, et entaille le lœess sur $20 \mathrm{~cm}$ de profondeur. Son comblement est homogène et consiste en un limon cendreux brun-noir associé à une forte densité de charbons de bois. Des graines carbonisées en quantité importante ont été observées, sans toutefois témoigner d'une fonction de stockage de cette structure : comme on le verra, la composition de cet assemblage, mêlant des céréales qui ne requièrent pas les mêmes traitements et dont certaines apparaissent moins nettoyées que d'autres, est le reflet de différents processus antérieurement à leur rejet dans la fosse.

La fosse a donné lieu à une datation qui situe son fonctionnement probablement au début du Chasséen : 5315 \pm 40 BP, soit [4311-4004] av. J.-C. cal.

\section{- La structure $34^{6}$}

De plan légèrement subcirculaire, elle présente un profil trapézoïdal et un fond plat assis sur le lœss carbonaté.

6. Anciennement $n^{\circ} 103$ du diagnostic. 
Figure 16 : Démouville « Le Clos Neuf $n$ : mobilier en hématite oolithique.

Figure 16: Démouville "ZAC du Clos Neuf", artefacts in oolithic hematite.
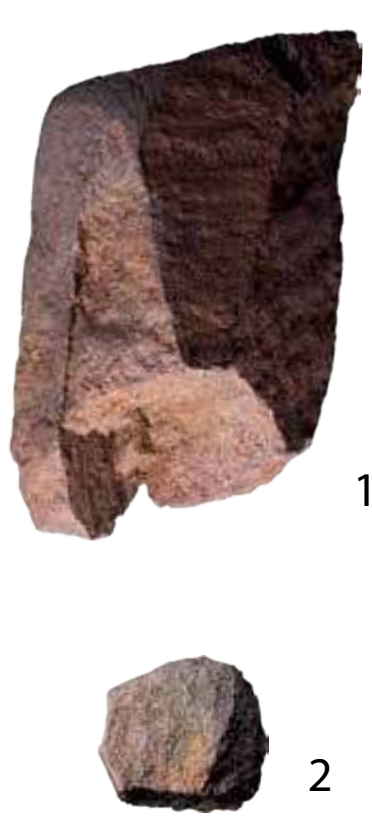

St. 86 , US $161-9$

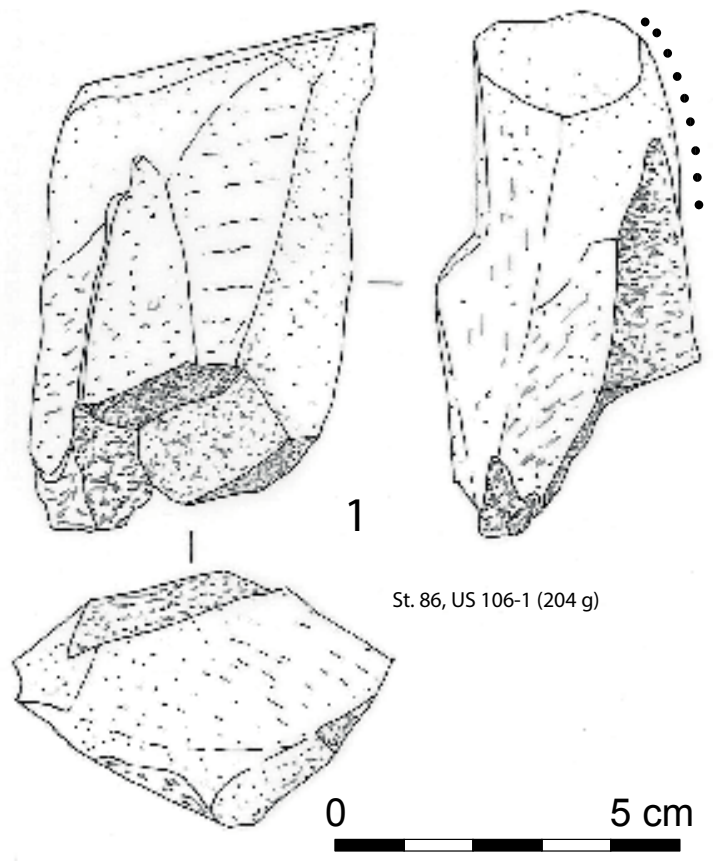

- La structure de combustion 67

La structure 67 se présente comme une fosse circulaire de 2,20 $\mathrm{m}$ de diamètre, pour une profondeur conservée de $0,20 \mathrm{~m}$. Les parois observent un profil vertical et le fond majoritairement plat montre une légère déclinaison au centre. Trois remplissages différents ont été observés : le premier, qui constitue le premier remplissage de la fosse (US 208.2) est un limon argileux induré de couleur brun foncé à rougeâtre, fortement rubéfié par endroits.

Quelques inclusions charbonneuses parsèment la matrice. À l'ouest de la fosse, un horizon plus compact observé sur une faible portion pourrait correspondre à une paroi du four ou une éventuelle sole. Aucun mobilier susceptible d'éclairer la fonction de cette structure (foyer alimentaire ou autre?) n'était inclus dans les différentes couches. Elle a bénéficié d'une datation par radiocarbone $(5795 \pm 40 \mathrm{BP}$, soit [4767-4541] av. J.-C. cal.) qui permet de la placer probablement à la fin du BVSG ou au début du Cerny.

\section{Le mobilier céramique du Néolithique moyen}

\section{- Présentation générale}

La fouille de l'unique comblement de la fosse 39 a permis de découvrir 81 tessons de poterie $(301,3 \mathrm{~g}$; tabl. 3 et fig. 19). Ils sont très fragmentés ( $4 \mathrm{~g}$ par tesson) et quelques surfaces sont érodées par des radicelles. Le nombre minimum d'individus est de trois vases pour un nombre maximum d'individus estimé à cinq. Aucun décor n'est identifié dans ce lot.
Il s'agit d'une fosse circulaire d'environ $1,50 \mathrm{~m}$ de diamètre, à profil resserré. La fosse a livré un ensemble conséquent de tessons de céramique appartenant à une écuelle carénée. 


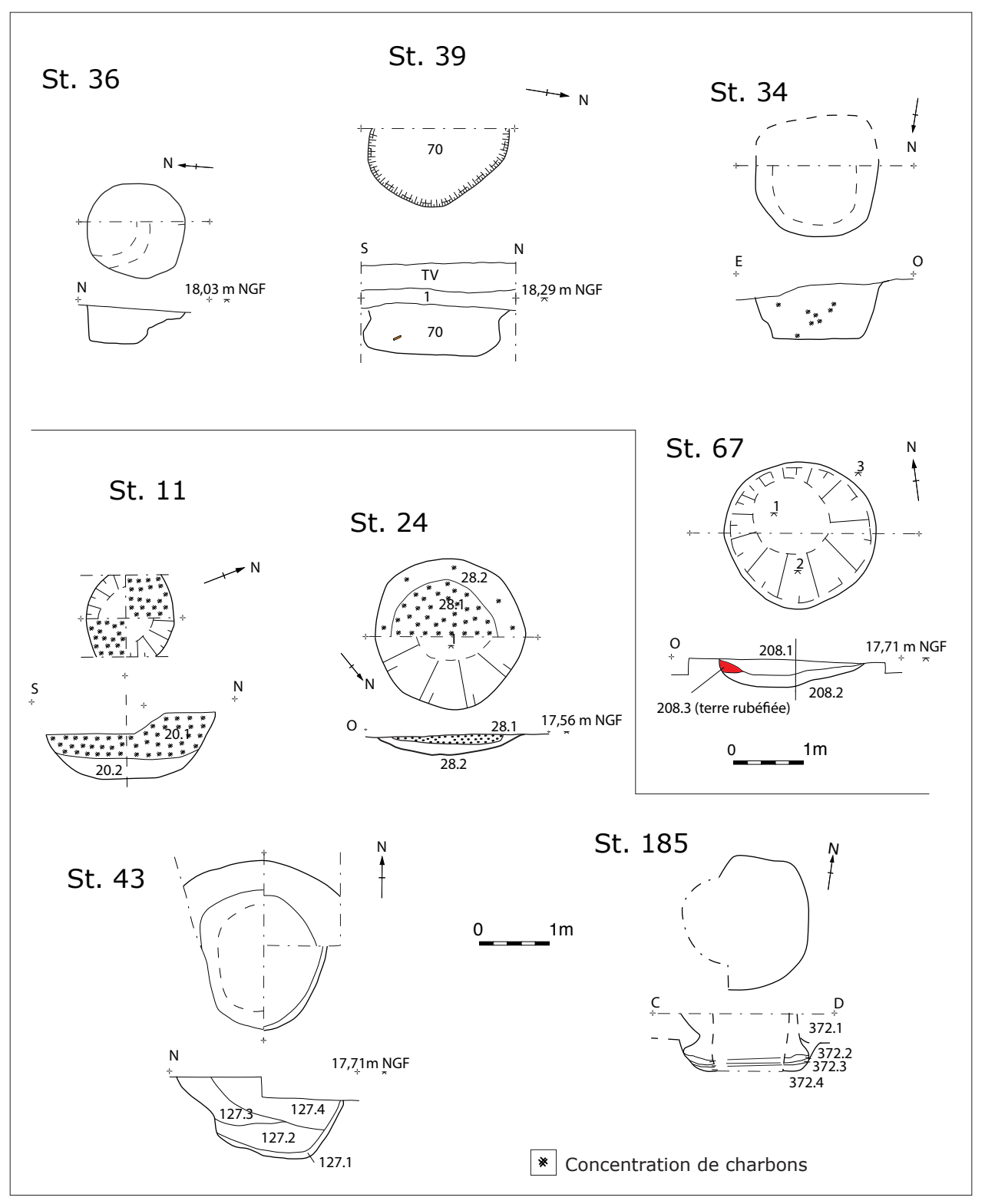

Figure 17 : Plans et coupes des autres structures attribuées au Néolithique : en haut, structures attribuées au Néolithique moyen ; en bas, structures probablement néolithiques sans attribution précise.

Figure 17: Plans and sections of the other features dating to the Neolithic; above, features dating to Middle Neolithic; below, features probably dating to the Neolithic, without precise chronology.

\section{- Les techniques de fabrication}

La détermination des inclusions visibles dans les poteries a permis de définir au moins trois groupes macroscopiques de terre argileuse. Une céramique vacuolaire se distingue par une forte porosité associée à de très rares inclusions. La terre mise en œuvre pour la confection de ce vase pourrait être une marne bioclastique disponible dans la Plaine de Caen. Une autre poterie est façonnée à partir d'une terre argileuse à glauconie. Elle renferme de nombreuses glauconies associées à quelques quartz arrondis à subanguleux. Ces terres argileuses ont une origine qui se situerait plutôt dans le Pays d'Auge, à l'est du Calvados. Les autres poteries renferment de nombreux quartz et feldspaths arrondis et anguleux associés à de nombreux gros micas blancs et fines paillettes de micas

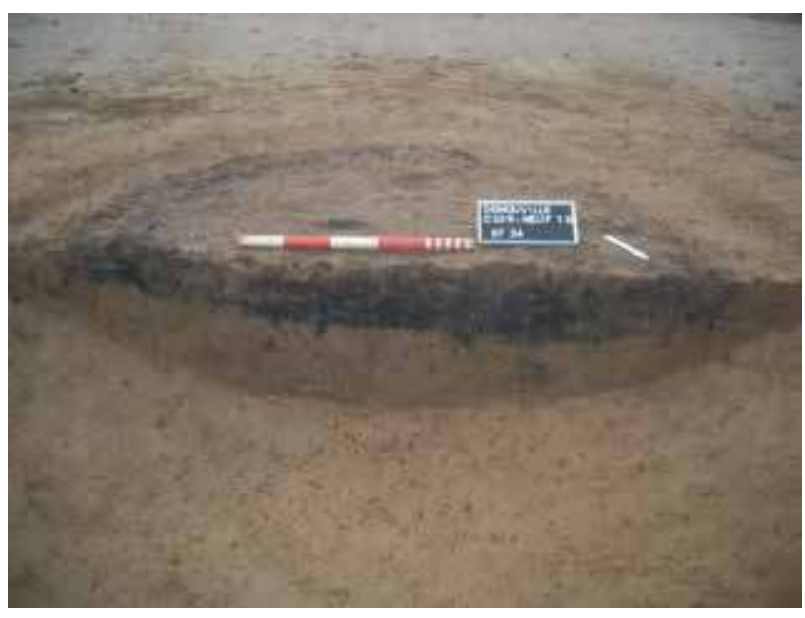

Figure 18: Vue et coupe de la st. 24.

Figure 18: View and section 24. 
(muscovites). Un dégraissant végétal est possiblement ajouté dans la pâte de la céramique vacuolaire.

Le montage au colombin est attesté par l'orientation horizontale des cassures. Les surfaces des récipients sont bien lissées. Une poterie a des surfaces externes possiblement polies (39.3). L'épaisseur des parois varie de 4 à $7 \mathrm{~mm}$. Les surfaces ont des couleurs brun orangé à noir et le cœur est généralement gris à noir.

\section{- Les formes et les éléments de préhension-suspension}

Deux individus correspondent à des formes basses à profil segmenté ouverte et fermée (fig. 19: 39.1 et 39.3). Ces formes ont de larges cols évasés surmontés par des lèvres amincies et arrondies. Leur diamètre à l'ouverture est d'environ 18 et $19 \mathrm{~cm}$. Elles ont des épaisseurs de $5 \mathrm{~mm}$. Ces formes ne sont pas décorées. Un individu est reconnu à partir d'un élément de préhension-suspension (fig. 19 : 39.2). Il est fragmenté et n'est par conséquent pas identifiable.

\section{- Attribution chronoculturelle et comparaisons}

Régionalement, l'individu 39.3 est comparable à un vase mis au jour sur le site du Néolithique moyen II de Cairon "La Pierre Tourneresse " (Calvados; Ghesquière et Marcigny, 2011 : fig. 109). D'autres comparaisons extrarégionales peuvent être mentionnées. Plusieurs formes semblables à l'individu 39.1 ont été découvertes sur le site chasséen de La Celle-Saint-Avant "Le Carroi Potet " (Indre-et-Loire; Lethrosne, 2016 : fig. 4). Les formes de Démouville sont également comparables à celles mises au jour dans les couches D du site Chasséen de Bouryen-Vexin «Le Cul-froid»(Oise; Martinez, 1991). Ces quelques comparaisons s'accordent pour dater la fosse st. 39 de Démouville au Néolithique moyen II.

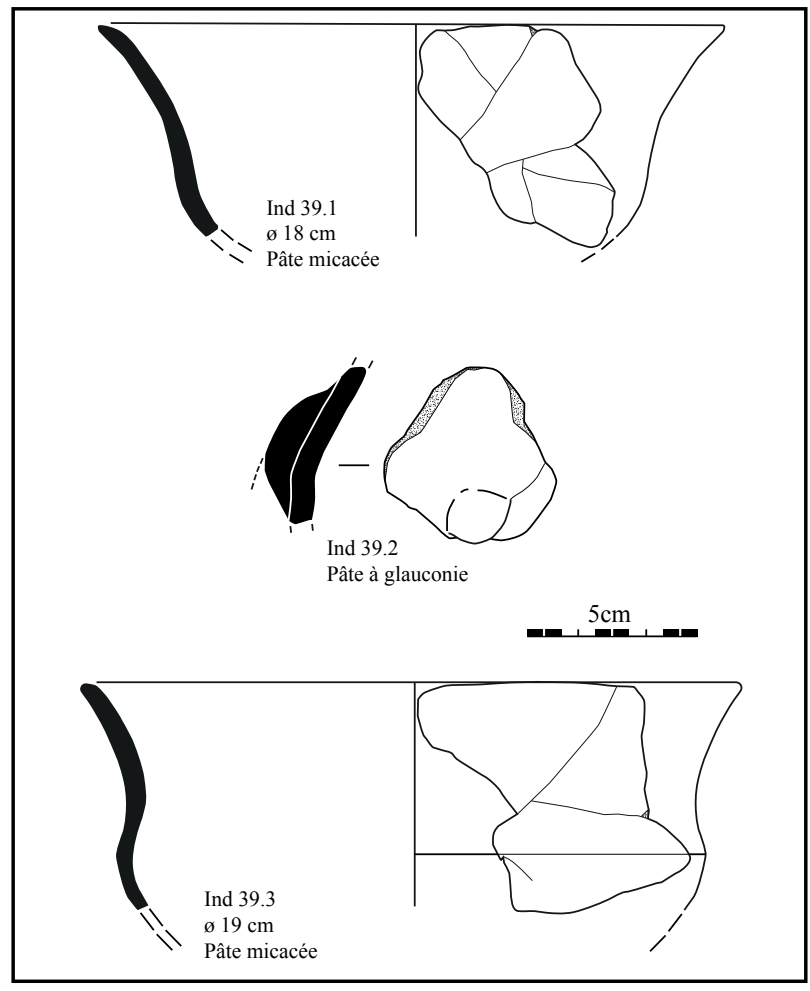

Figure 19: Démouville «ZAC du Clos Neuf » : mobilier céramique de la structure 39.

Figure 19: Démouville "ZAC du Clos Neuf”: pottery from the structure 39.

\section{Le mobilier lithique du Néolithique moyen II}

L'ensemble des structures attribuées au Néolithique moyen a livré un effectif relativement réduit de matériel lithique (tabl. 4) : au total 230 objets en silex, dont une forte proportion de débris (155 débris, soit $68 \%$ ); 56 déchets de taille (24\%) et seulement 19 outils ( $8 \%$ ). L'approvisionnement en silex est diversifié. Il recourt à une variété de ressources régionales visiblement apportées

\begin{tabular}{|c|c|c|c|c|c|c|c|c|c|c|c|c|}
\hline St & Ind. & Nr & PNr g. & B & D & $\mathbf{P}$ & Relief & Aspect & Ep. mm & S Ext./Cour/ S Int. & Pâte & Observation \\
\hline 39 & 39.1 & 34 & 125,9 & 1 & - & - & Rugueux & Mat & $5 \mathrm{~mm}$ & $\begin{array}{c}\text { Brun orangé/Noir/ } \\
\text { Brun orangé }\end{array}$ & Micacée & 1 ind $4 \mathrm{~B}$ \\
\hline & 39.2 & 4 & 32 & - & - & 1 & Lisse & Mat & $7 \mathrm{~mm}$ & Brun beige/Gris/Gris & Glauconie & \\
\hline & 39.3 & 29 & 106,3 & 1 & - & - & Lisse & Brillant & $5 \mathrm{~mm}$ & Noir/Noir/Noir & Micacée & 1 ind 2B \\
\hline & 39.4 & 11 & 32 & - & - & - & Lisse & Mat & $4 \mathrm{~mm}$ & $\begin{array}{c}\text { Brun orangé/Noir/ } \\
\text { Noir }\end{array}$ & Micacée & \\
\hline & 39.5 & 2 & 1,2 & - & - & - & $\begin{array}{c}\text { Lisse } \\
\text { bosselé }\end{array}$ & Mat & $5 \mathrm{~mm}$ & $\begin{array}{c}\text { Beige orangé/Gris } \\
\text { noir/Gris noir }\end{array}$ & Vacuolaire & Noir gris/Orange/ \\
Noir gris & & - & Historique \\
\hline
\end{tabular}

Tableau 3 : Données quantitatives et pondérales du mobilier céramique de la fosse 39. 

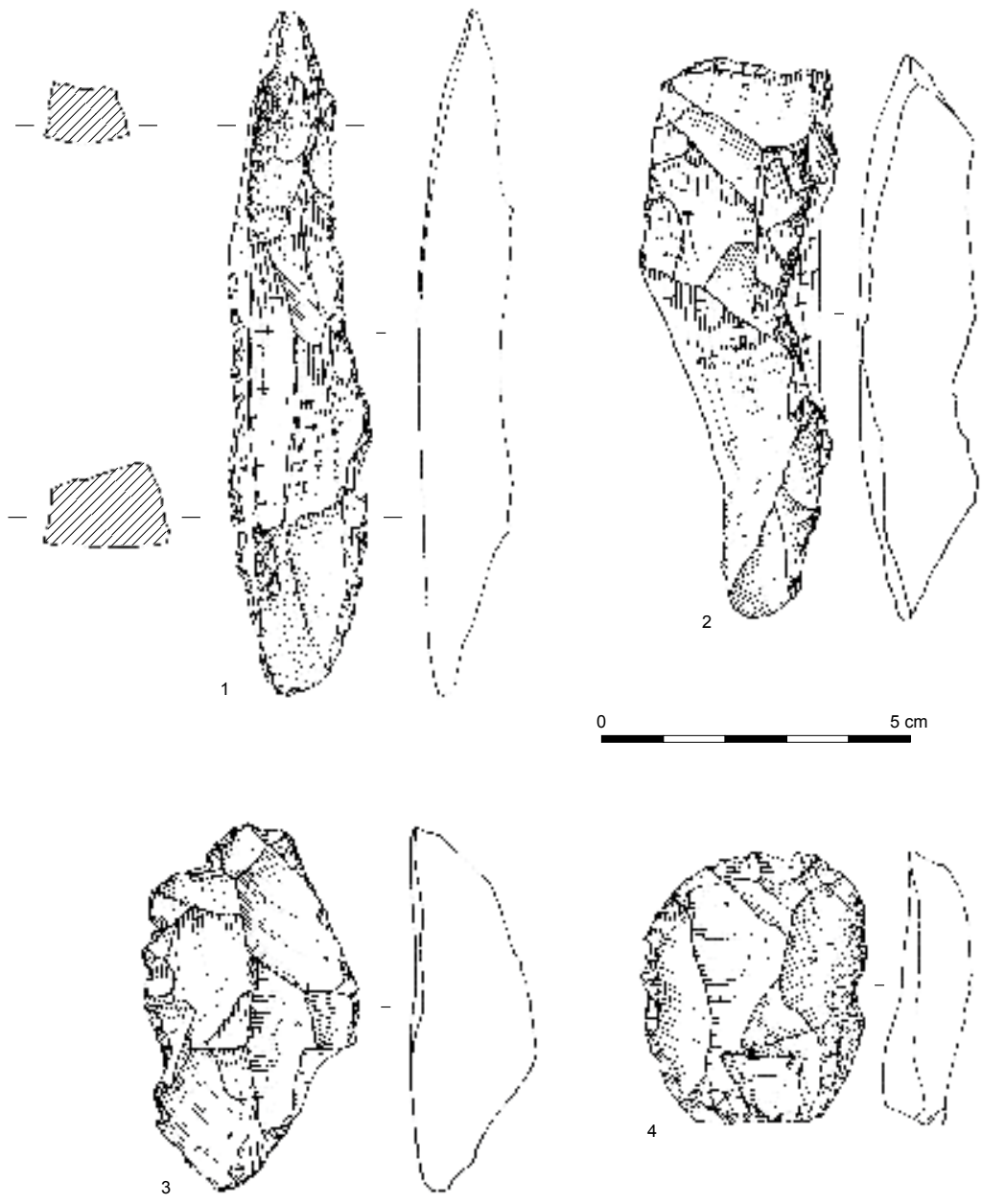

Figure 20 : Démouville « Le Clos Neuf » : mobilier lithique du Néolithique moyen II.

Figure 20: Démouville "ZAC du Clos Neuf": Middle Neolithic Il, lithics. brutes et entièrement débitées sur place. Quelques matériaux ont visiblement été apportés depuis le littoral. Le silex du Cinglais est toujours fortement représenté (deux tiers de la série), mais l'exigence de qualité des blocs n'est pas essentielle dans le cadre du débitage réalisé au Néolithique moyen. Les déchets de taille témoignent en effet d'un unique schéma opératoire simple de production d'éclats, non prédéterminé, entièrement réalisé par percussion directe dure selon un schéma multidirectionnel. Outre neuf éclats de dégrossissage, on trouve trente-huit éclats bruts, dont dix-huit allongés et vingt courts, pour un seul nucléus. L'outillage est également très réduit pour cette phase d'occupation : sur les dix-neuf outils présents, les seuls outils typologiquement identifiés sont sept grattoirs sur éclat (fig. 20), outils ubiquistes et sans signification chronotypologique, et un petit pic en silex (st. 36, fig. 20). Cet objet peut être mis en rapport avec le creusement des fosses circulaires. Le reste de l'outillage ne présente pas de caractères particuliers : il s'agit essentiellement d'outils expédients sur éclats, notamment sept éclats retouchés ou écaillés (fig. 20) et deux éclats denticulés (fig. 20). Quelques éléments laminaires sont présents dans les struc- tures du Néolithique moyen : il s'agit de quatre fragments de lames en silex du Cinglais et d'une petite lame écaillée. Ces objets sont sans doute des éléments de l'occupation du Néolithique ancien repris dans les terres de comblement des structures ultérieures. Pour le reste, les rares éléments typologiques présents ne permettent pas une comparaison pertinente avec les autres ensembles du Néolithique moyen dans la région.

\begin{tabular}{|c|c|}
\hline Déchets de taille & \\
\hline Éclat de dégrossissage & 9 \\
\hline Fragment de lame & 4 \\
\hline Éclat allongé brut & 18 \\
\hline Éclat court brut & 20 \\
\hline NE multidirectionnel & 1 \\
\hline Total débitage & 56 \\
\hline Débris & 139 \\
\hline Esquille & 16 \\
\hline Casson d'éclat & 4
\end{tabular}




\begin{tabular}{|c|c|}
\hline Total débris & $\mathbf{1 5 5}$ \\
\hline Outillage & \\
\hline Grattoir sur éclat & 7 \\
\hline Pic & 1 \\
\hline Ébauche de tranchet & 1 \\
\hline Éclat retouché/écaillé & 7 \\
\hline Éclat denticulé & 2 \\
\hline Lame écaillée & 1 \\
\hline Total outillage & $\mathbf{1 9}$ \\
\hline Total série & $\mathbf{2 3 0}$ \\
\hline
\end{tabular}

Tableau 4 : Décompte de l'industrie lithique des structures du Néolithique moyen.

Table 4: Lithic counts from the Middle Neolithic features.

Une petite hache polie en dolérite a également été découverte dans la structure 34 (fig. 21). Elle mesure $8 \mathrm{~cm}$ de long et $4 \mathrm{~cm}$ de large. Ses faces et ses flancs sont entièrement polis. Le tranchant présente un net émoussé et quelques ébréchures rapportables à une utilisation. En particulier, quelques écaillures unifaciales du tranchant, associées à des traces d'impact sur le talon de la pièce, signalent potentiellement une utilisation de l'objet en percussion indirecte, à la manière d'un ciseau, sans doute à l'occasion d'un réemploi. Une autre hache polie d'un module comparable a été découverte hors contexte, mais en surface de décapage à proximité de la st. 34 (fig. 21). Elle est également en dolérite. La longueur conservée de la pièce est de $6,5 \mathrm{~cm}$, pour une largeur maximale de $3,7 \mathrm{~cm}$. Son état de surface laisse apparaitre un traitement moins poussé que pour l'exemplaire de la structure 34 (fig. 21) : ses flancs présentent encore des traces de bouchardage. L'état des surfaces polies varie également, entre les zones proches du talon de la hache, sur les faces et les facettes partant du tranchant. Les flancs et les faces sont traités à l'aide d'un abrasif à grain épais. Les facettes proches du tranchant révèlent sans nul doute un raffûtage réalisé à l'aide d'un abrasif fin. Le tranchant originel poli n'est pas conservé : il est écaillé et emporté par deux enlèvements qui couvrent une part importante de la surface d'une des faces de la pièce. Les arêtes émoussées de cette face semblent indiquer une ultime tentative de repolissage avant abandon.

\section{Les autres structures probablement néolithiques $(2,18,23,41,43,68,78,167$ et 185) (fig. 17)}

\section{- La structure de combustion 18}

Une structure de combustion a été détectée au nordouest, accolée à la berme ouest du site. Aucun creusement n'était perceptible et les seuls témoins d'une activité terre rubéfiée irrégulière, mal conservée, sur $1 \mathrm{~cm}$ d'épaisseur. Il s'agit d'un épandage de terre cuite architecturale qui pourrait relever d'un possible reliquat de sole.

\section{- La structure 23}

La structure 23, la plus septentrionale, se présente comme une fosse de plan circulaire de $1,40 \mathrm{~m}$ de diamètre pour $0,35 \mathrm{~m}$ de profondeur conservée. Son profil est plutôt évasé et son fond plat. Deux types de remplissage ont été distingués : le premier, affectant le fond de la fosse, est un niveau charbonneux de quelques centimètres d'épaisseur, comportant de nombreuses traces de rubéfaction. Le remplissage supérieur est composé d'un limon brun grisâtre moyen contenant également quelques résidus de combustion. Un fragment de panse à pâte sombre et deux micro-esquilles osseuses calcinées sont apparus dans son comblement.

\section{- La structure 41}

Cette fosse subcirculaire a livré un fragment de fond rond qui autorise une attribution au Néolithique ancienmoyen sans plus de précision. L'une de ses US très organique (106-2) a également fourni quelques dents de bovinés (Bos taurus), un fragment d'os spongieux brûlé et quelques éclats de silex.
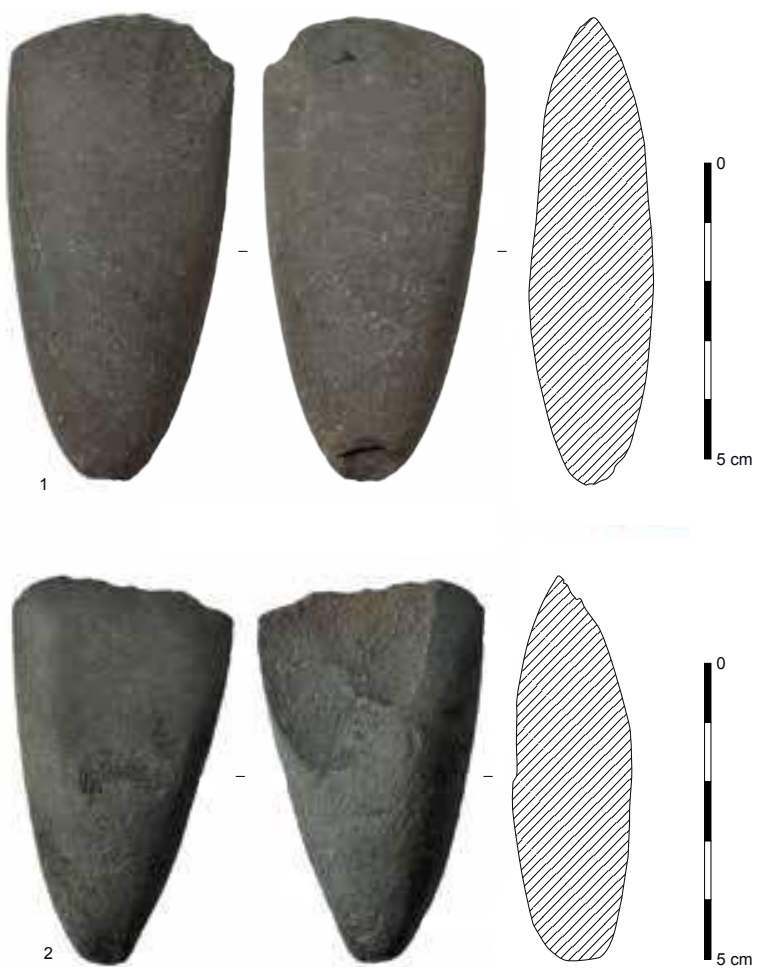

Figure 21 : Démouville "Le Clos Neuf » : outillage poli du Néolithique moyen II.

Figure 21: Démouville "ZAC du Clos Neuf”: Middle Neolithic II polished tools. 


\section{- La structure 43 (fig. 22)}

Située à l'est du site, dans un alignement géographique avec le regroupement de silos précédemment évoqué, la structure 43 se présente comme une fosse de plan circulaire aux parois verticales s'évasant sur sa partie supérieure. Le fond est presque plat et entaille partiellement le substrat calcaire. Ses dimensions sont importantes : elle affecte 2,5 $\mathrm{m}$ de diamètre à l'ouverture pour $1,1 \mathrm{~m}$ de profondeur. Quatre phases de remplissage ont été observées (de bas en haut) :

- I'US 127.1, tapissant le fond de la fosse sur une dizaine de centimètres, se présente comme un limon sableux brunjaune moyen, de consistance semi-compacte. Cet horizon semble agir comme une interface entre le loess carbonaté encaissant et le remplissage effectif de la structure.

- l'US 127.2, un limon argileux brun foncé à consistance semi-compacte, a livré quelques fragments d'une céramique délitée ou de terre cuite architecturale ainsi que de rares charbons de bois.

- l'US 127.3 s'en distingue par une couleur jaune foncé incluant quelques charbons. Cette couche reconnue sur la paroi nord de la structure résulte probablement de l'effondrement des parois de la structure lors de son fonctionnement à ciel ouvert.

- l'US 127.4, ultime remplissage de la fosse, présente à nouveau dans sa matrice limoneuse une densité de charbon notable, évoquant un rejet de foyer.

Malgré ces caractéristiques et en l'absence de graines, cette fosse ne peut être qualifiée de structure d'ensilage.

\section{- La structure 68}

Située à côté de la structure précédente, elle se présente comme une fosse irrégulière de 3,08 $\mathrm{m}$ de long pour 1,46 m de large. Elle présente une orientation nord-sud. Sa forme en plan est très irrégulière et comprend grossièrement deux lobes. Deux couches la caractérisent : le remplissage primitif de la fosse comprend quelques inclusions charbonneuses disséminées dans la matrice limoneuse. Dans sa partie nord, on observe une poche brun orangé foncé d'un diamètre d'1 $\mathrm{m}$. Elle n'est conservée que sur quelques centimètres et relève sans doute d'un rejet de foyer provenant de la vidange de la structure de combustion 67 voisine.

\section{- La structure 185}

Détectée à l'ultime phase de la campagne de fouille, la fouille de cette structure illustre les difficultés de lecture inhérentes à la nature du substrat. En effet, seule une tache circulaire évoquant un possible trou de poteau a tout d'abord été perçue dans le substrat limoneux. Un décaissement mécanique complémentaire a finalement révélé une fosse nettement plus imposante, d'environ 1,70 à $1,80 \mathrm{~m}$ d'envergure pour une profondeur conservée de $0,80 \mathrm{~m}$ sous la terre végétale et l'horizon limoneux, probablement un silo.

Contrairement aux autres fosses, cette structure affecte un profil bitronconique très nettement dessiné, avec une partie supérieure évasée, un étranglement et une partie inférieure globulaire. De même, les différentes strates observées témoignent d'une bonne conservation de la structure :

- l'US 372.5 est la couche de lœess carbonaté naturel dans lequel s'est probablement inscrit le creusement de la structure, reposant elle-même sur le substrat calcaire;

- constituant la paroi du silo, l'horizon 372.4 se compose d'une bande irrégulière d'environ $5 \mathrm{~cm}$ d'épaisseur, fortement rubéfiée et charbonneuse, qui pourrait résulter d'un assainissement de la structure par le feu;

- cet horizon est surmonté d'un limon argileux sombre, extrêmement induré et riche en charbons (US 372.3). Quelques graines carbonisées ont pu être observées dans la matrice limoneuse, mais cette structure n'a pas fait l'objet d'un échantillonnage carpologique.

Une couche intermédiaire, observant le même " litage " horizontal, a été identifiée entre les horizons 372.3 et les horizons supérieurs. Le comblement supérieur affectant la structure est un horizon à matrice limoneuse brun moyen, d'une puissance de $60 \mathrm{~cm}$, comportant quelques traces de charbons et de rares silex. On notera que du mobilier lithique a été retrouvé en relative abondance aux différents niveaux de remplissage de la structure.

\section{- Les trous de poteau}

Seules deux structures peuvent être assimilées à des trous de poteau, bien qu'isolées et localisées en dehors de tout ensemble organisé. La structure 2 affecte un plan ovoïde de $0,40 \mathrm{~m}$ de diamètre, un profil en cuvette

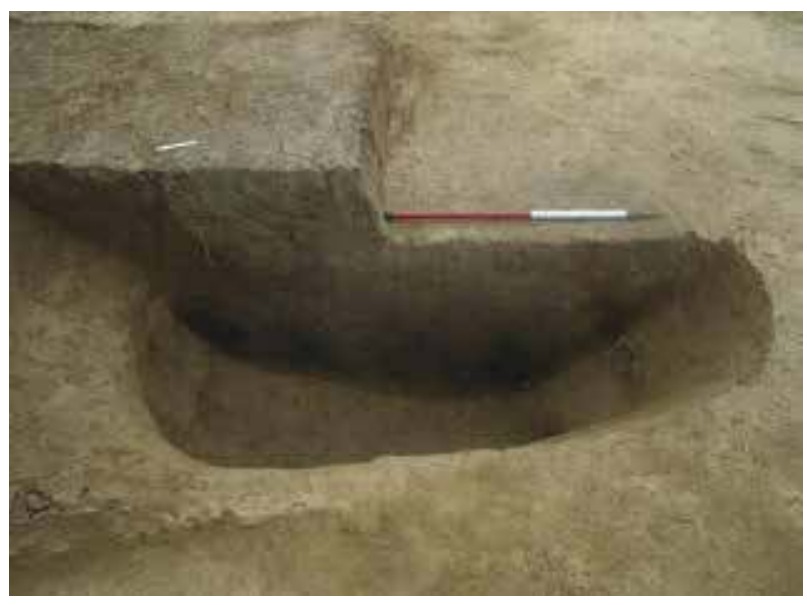

Figure 22 : Vue et coupe de la st. 43.

Figure 22: View and section 43. 
pour une profondeur de $0,38 \mathrm{~m}$. La structure 167 a été observée à proximité immédiate de la structure 26. Elle présente un plan circulaire de $0,45 \mathrm{~m}$ de diamètre et un profil en V. La matrice limono-argileuse de couleur brun foncé présentait quelques inclusions charbonneuses. $\mathrm{Ni}$ la stratigraphie relative ni aucun indice chronologique ne permet d'associer ces deux structures.

\section{LES MACRORESTES VÉGÉTAUX}

L'étude des macrorestes végétaux de Démouville « ZAC du Clos Neuf " avait été initiée par P. Marinval sous la forme d'un test destiné à estimer la richesse des différents prélèvements, puis abandonné. La présence de très nombreux macrorestes carbonisés dans certaines des structures échantillonnées nous a incités à reprendre cette étude.

\section{Matériaux et méthodes}

Les sédiments, issus d'une demi-douzaine de structures relevant des différentes occupations du site au Néolithique (voir infra), avaient été flottés sur trois tamis calibrés aux mailles de $4 \mathrm{~mm}, 2 \mathrm{~mm}$ et $0,5 \mathrm{~mm}$ d'ouverture. L'absence d'éléments grossiers explique que la plupart des échantillons n'ont pas généré de refus de $4 \mathrm{~mm}$, ce qui importe peu pour notre étude. En revanche, il était primordial de disposer des deux refus plus fins $(2 \mathrm{~mm}$ et $0,5 \mathrm{~mm}$ ), exigence respectée dans presque tous les cas. La seule exception réside dans le cas de la structure 11. Le tableau fourni avec les refus de flottation à étudier stipulait l'absence de refus de $2 \mathrm{~mm}$. Nous nous sommes toutefois demandé s'il en manquait vraiment un, et si tel était le cas, s'il ne s'agissait pas plutôt de celui de $0,5 \mathrm{~mm}$ dans la mesure où nous n'avons pas isolé de semences de petites dimensions.

Tous les refus de flottation ont été intégralement triés, sauf celui de $2 \mathrm{~mm}$ de la fosse 24. Dans ce dernier cas, seul $300 \mathrm{ml}$ de refus de tamis ont été triés, soit environ la moitié du volume total du refus.

Le tableau 5 mentionne les échantillons étudiés, leur origine au sein de chaque structure, le volume traité, généralement égal à 40 litres par US, sauf dans le cas de la fosse rubanée 86. Au sein de l'US 161-3, le volume du prélèvement s'élève à 50 litres et au sein de l'US 1615, deux prélèvements ont été extraits, ce qui monte à 80 litres le volume total de sédiment étudié pour cette US.

Les sept structures néolithiques sur lesquelles porte cette étude consistent en :

- deux fosses (st. 86 et st. 26) attribuées au Rubané. Les six échantillons qui en proviennent totalisent un volume de 250 litres de sédiment;
- quatre fosses (st. 24, st. 34, st. 11, st. 67) attribuées au Néolithique Moyen II. Les quatre échantillons étudiés représentent un volume total de 200 litres;

- une structure de combustion (st. 23) qui ne peut être rapportée à l'une ou l'autre période d'occupation néolithique. L'échantillon qui en est issu possède un volume de 40 litres de sédiment.

L'origine des échantillons au sein des différentes structures sera précisée lors de la présentation des données carpologiques recueillies pour les différentes périodes d'occupation. Les résultats chiffrés figurent au sein des tableaux 2 et 3 , respectivement pour les structures du Néolithique ancien et du Néolithique moyen II. Ces résultats sont exprimés en NMI, obtenus en divisant par deux le nombre de fragments de grains de céréales identifiées comme blés ou orges et les semences d'herbacées sauvages, et par quatre les fragments de grains de céréales indéterminées (Cerealia) et de coquilles de noisettes (Corylus avellana). Les résultats concernant les vannes de céréales (furcas, rachillets et bases de glumes de blés vêtus, fragments de rachis de blé nu) sont exprimés en nombre de restes. Le sous-échantillonnage appliqué au refus de flottation de $2 \mathrm{~mm}$ de la fosse 24 a conduit à pondérer les résultats obtenus (tabl. 5 et 7).

\section{Composition générale et distribution des macrorestes}

Sur l'ensemble des structures étudiées, le tri des refus de flottation a généré 13216 macrorestes végétaux, tous carbonisés. La majorité d'entre eux (8398, soit 63,55 \%) provient des structures du NMII, dans une moindre mesure, des fosses rubanées (4816, soit 36,44\%) et enfin de la structure de combustion 23 dont l'appartenance à l'une ou l'autre période d'occupation au sein du Néolithique n'a pu être précisée. Cette structure ne livre qu'un seul grain de céréale, attribuable à l'orge nue (Hordeum vulgare var. nudum).

Le spectre taxonomique est globalement riche de six plantes cultivées et de douze plantes sauvages, dont sept identifiées au rang spécifique.

\section{Les macrorestes végétaux associés à l'occupation rubanée}

Les deux fosses rubanées ne s'avèrent pas aussi riches d'informations l'une que l'autre. Bien qu'un important volume de sédiment en ait été extrait et traité, la première, st. 86, ne fournit que huit restes au total, dont une majorité de fragments de coquilles de noisette (Corylus avellana). Le seul grain de céréale identifié provient vraisemblablement d'un blé nu (Triticum cf. aestivum/turgidum/durum). 
La fosse st. 26, de forme ovoïde, appartient peutêtre à une autre unité d'habitation que la précédente. L'échantillon étudié provient de la couche supérieure de son comblement, couche que les fouilleurs décrivent comme un limon argileux densément fourni en charbons et graines carbonisées. Si l'on rapporte le nombre de macrorestes carbonisés qui en ont été extraits (4808), au volume de sédiment traité (40 litres), la densité obtenue, de 120 restes au litre, s'avère suffisamment élevée pour que l'on puisse parler de concentration de graines. Elle est toutefois bien inférieure à ce que fournirait un assemblage en position primaire, interprétation à laquelle s'oppose d'ailleurs son origine spatiale au sein de cette fosse.

Cet assemblage est essentiellement constitué de restes de plantes cultivées $(98,6 \%)$. Il s'agit principalement de céréales - orge vêtue (Hordeum vulgare, fig. 23, n 1 ), amidonnier (Triticum dicoccum, fig. 23, $\mathrm{n}^{\circ} 2$ ), engrain (Triticum monococcum, fig. 23, ${ }^{\circ} 3$ et 4), blé nu (Triticum aestivum/turgidum/durum, fig. $\left.23, \mathrm{n}^{\circ} 5\right)$ - auxquelles s'ajoute le pavot (Papaver somniferum), sous la forme d'une demi-douzaine de ses semences oléagineuses carbonisées (fig. 23, $\mathrm{n}^{\circ} 8$ ). Dans l'ensemble, les restes céréaliers consistent majoritairement en grains $(97,4 \%$ ), laissant peu de place aux sous-produits de leur traitement - vannes et semences d'herbacées sauvages - dans la composition de cet assemblage, à laquelle elles contribuent équitablement à hauteur de 1,3\% (fig. 24). Dans le détail, l'orge vêtue, céréale très nettement prédominante (67\%) (fig. 25) ne livre aucun article de rachis et ses grains apparaissent débarrassés de leurs enveloppes. En ce qui concerne les blés, la même impression de grains nettoyés peut être déduite de l'absence de rachis de blé nu et de la faible représentation des vannes de blés vêtus, autrement dit de furcas et bases de glumes d'amidonnier et d'engrain (fig. $23, n^{\circ} 6$ ), en regard des grains que fournissent ces derniers. Amidonnier et engrain représentent respectivement $16 \%$ et $7 \%$ des restes céréaliers, le blé nu $10 \%$ (fig. 26). Il est probable que toutes les semences d'herbacées sauvages - renouée liseron (Fallopia convolvulus, fig. 23, $\mathrm{n}^{\circ}$ 9), lampsane commune (Lapsana communis, fig. $23, \mathrm{n}^{\circ} 10$ ), chénopode blanc (Chenopodium album), gaillet gratteron (Galium aparine), pâturin annuel (Poa annnua), vesce hérissée (Vicia hirsuta) - soient celles d'adventices des cultures, dans la mesure où elles font partie du cortège des pestes des récoltes habituellement associées aux assemblages de céréales dès le Rubané. Néanmoins, s'agissant de grains en position secondaire, il n'est pas non plus exclu que certaines de ces semences, surtout celles de plantes au statut plus ambigu - tels la lampsane, fréquente en lisière de forêt, le gaillet gratteron, commun dans les terrains embroussaillés ou le pâturin annuel, auquel les sols piétinés des abords des habitations ou des chemins fournissent de bonnes conditions de développement - se soient trouvées mêlées aux grains, sans toutefois provenir des mêmes parcelles cultivées. Quelle que soit l'hypothèse retenue, la présence de ces herbacées parmi les grains de céréales est des plus ténues (voir supra). Il s'agirait donc de récoltes prêtes à être consommées et on peut suggérer leur chute accidentelle dans une même structure de combustion dévouée à leur préparation, les grains d'orge vêtue figurant le dernier " repas ", ceux des autres céréales, des préparations antérieures, l'ensemble mêlé aux charbons de bois également nombreux dans le comblement de cette US.

À elle seule, la fosse 26 de Démouville livre davantage de macrorestes végétaux que les 16 fosses échantillonnées à Colombelles «Le Lazarro " (Dietsch-Sellami, 2014). Le cortège des céréales mises en culture est identique sur les deux sites, aux occupations, semble-t-il, assez proches

\begin{tabular}{|c|c|c|c|c|c|c|c|}
\hline Occupations & $\mathbf{N}^{\mathbf{0}}$ structure & $\mathbf{N}^{\mathbf{P V}}$ & $\mathbf{U S}$ & Volume & $\mathbf{4} \mathbf{~ m m}$ & $\mathbf{2} \mathbf{~ m m}$ & $\mathbf{0 , 5} \mathbf{~ m m}$ \\
\hline Rubane் & St 86 & 17 & $161-3$ & 501 & TI & TI & TI \\
\hline & St 86 & 18 & $161-5$ & 401 & Abs. & TI & TI \\
\hline & St 86 & 19 & $161-5$ & 401 & Abs. & TI & TI \\
\hline & St 86 & 20 & $161-6$ & 401 & Abs. & TI & TI \\
\hline & St 86 & 21 & $161-7$ & 401 & Abs. & TI & TI \\
\hline & St 26 & 6 & $75-1$ & 401 & Abs. & TI & TI \\
\hline NMII & St 34 & 4 & 56 & 401 & Abs. & TI & TI \\
\hline & St 11 & 1 & $20 / 01 / 20$ & 401 & TI & TI & $?$ \\
\hline & St 24 & 3 & $28 / 01 / 20$ & 401 & Abs. & TP & TI \\
\hline & St 67 & 30 & $208-1$ & 401 & Abs. & TI & TI \\
\hline Néolithique & St 23 & 25 & 219 & 401 & Abs. & TI & TI \\
\hline
\end{tabular}

Tableau 5 : Liste des structures étudiées à Démouville « ZAC du Clos Neuf » (Calvados) (TI : tri intégral ; TP : tri partiel).

Table 5: List of studied features from Démouville "ZAC du Clos Neuf” (Calvados) (TI: integral sample; TP: partial sample). 


\begin{tabular}{|c|c|c|c|c|c|c|c|}
\hline & Structure & & 26 & 86 & 86 & 86 & 86 \\
\hline Démouville & Échantillon & & 6 & 17 & 18 & 19 & 21 \\
\hline \multirow[t]{2}{*}{ Néolithique ancien } & US & & 75.1 & 161.3 & 161.5 & 161.5 & 161.7 \\
\hline & Litrage & & 401 & 501 & 401 & 401 & 401 \\
\hline \multirow[t]{6}{*}{ Céréales, grains } & Hordeum vulgare & & 3110 & * & * & * & * \\
\hline & Triticum aestivum/turgidum/durum & & 464 & * & $*$ & * & * \\
\hline & Triticum cf. aestivum/turgidum/durum & & $*$ & * & * & $*$ & 1 \\
\hline & Triticum dicoccum & & 708 & * & * & * & * \\
\hline & Triticum monococcum & & 322 & * & * & * & * \\
\hline & Cerealia & & 59 & 1 & * & * & * \\
\hline \multirow[t]{6}{*}{ Céréales, vannes } & Triticum aestivum/turgidum/durum & fgts rachis & 2 & * & $*$ & * & * \\
\hline & Triticum dicoccum & furcas & 3 & * & $*$ & * & $*$ \\
\hline & Triticum dicoccum & bases glumes & 43 & * & * & * & $*$ \\
\hline & Triticum dicoccum & rachillet & 1 & * & * & * & * \\
\hline & Triticum monococcum & furcas & 3 & * & * & * & $*$ \\
\hline & Triticum monococcum & bases glumes & 13 & $*$ & * & * & $*$ \\
\hline Oléagineuse & Papaver somniferum & & 13 & $*$ & $*$ & * & $*$ \\
\hline \multirow[t]{15}{*}{ Flore sauvage } & Bromus sp. & & 1 & $*$ & * & * & $*$ \\
\hline & cf. Phleum & & 1 & * & * & * & $*$ \\
\hline & Chenopodium album & & 2 & * & * & * & $*$ \\
\hline & Corylus avellana & & 1 & 2 & 1 & 1 & $*$ \\
\hline & Fallopia convolvulus & & 50 & $*$ & $*$ & $*$ & $*$ \\
\hline & Galium aparine & & 1 & $*$ & * & $*$ & $*$ \\
\hline & Lapsana communis & & 5 & * & * & $*$ & $*$ \\
\hline & Poa annua & & 1 & $*$ & $*$ & $*$ & $*$ \\
\hline & Vicia hirsuta & & 2 & $*$ & * & * & $*$ \\
\hline & Vicia sp. petite & & 1 & $*$ & $*$ & * & $*$ \\
\hline & cf. Vicia & & $*$ & $*$ & * & 1 & * \\
\hline & Fabaceae & & 1 & * & * & * & * \\
\hline & cf. Fabaceae & & * & * & * & * & 1 \\
\hline & Indéterminé & & 1 & * & $*$ & $*$ & $*$ \\
\hline & Nombre total de restes & & 4808 & 3 & 1 & 2 & 2 \\
\hline
\end{tabular}

Tableau 6 : Décomptes des macrorestes du Néolithique ancien de Démouville « ZAC du Clos Neuf ».

Table 6: Early Neolithic macroremains from Démouville "ZAC du Clos Neuf".

dans le temps. La seule différence notable réside dans la présence du pois à Colombelles, celle du pavot somnifère à Démouville. Les commentaires engendrés par l'étude carpologique de Colombelles valent également à Démouville et concernent :

- la présence systématique de l'amidonnier et de l'engrain sur les sites rubanés (Bakels, 1979; 1984; 1991; Bakels et Rousselle, 1985; Heim, 1985; 1988).

- celle, beaucoup plus surprenante de l'orge, d'autant qu'il s'agit, sur l'un comme l'autre site, d'orge vêtue (Hordeum vulgare). Or les quelques mentions d'orge enre- gistrées en contexte rubané concernaient jusqu’à présent la variété à grains nus (Hordeum vulgare var. nudum). Cette dernière est attestée dans la vallée de l'Aisne, notamment à Menneville "Derrière le Village " (Bakels, 1984), à Berryau-Bac «Le Chemin de la Pêcherie » (Bakels, 1995a), ainsi qu’à Bucy-le-Long "La Fosse Tounise »/ « La Héronnière " (Bakels, 1995b). À propos de Menneville, C. Bakels souligne : "The presence of barley is remarkable. This cereal is usually missing in the Bandkeramik settlements from Central and Western Europe [...]. In France, the cereal was perhaps more often cultivated than in the adjacent regions. " Quant à 


\begin{tabular}{|c|c|c|c|c|c|}
\hline Démouville & Structure & & 24 & 34 & 11 \\
\hline & Échantillon & & 3 & 4 & 1 \\
\hline \multirow[t]{2}{*}{ Néolithique moyen II } & US & & 28.1 & 56 & 20.1 \\
\hline & Litrage & & 401 & 401 & 401 \\
\hline \multirow[t]{5}{*}{ Céréales, grains } & Hordeum vulgare nudum & & 2286 & * & 416 \\
\hline & Triticum aestivum/turgidum/durum & & 78 & $*$ & 196 \\
\hline & Triticum dicoccum & & 4200 & $*$ & 27 \\
\hline & Triticum monococcum & & 349 & * & 6 \\
\hline & Cerealia & & * & * & 6 \\
\hline \multirow[t]{6}{*}{ Céréales, vannes } & Triticum aestivum 1.s. & fgt rachis & 2 & $*$ & $*$ \\
\hline & Triticum dicoccum & furcas & 41 & $*$ & $*$ \\
\hline & Triticum dicoccum & bases glumes & 446 & $*$ & $*$ \\
\hline & Triticum dicoccum & rachillets & 46 & * & * \\
\hline & Triticum monococcum & furcas & 15 & * & $*$ \\
\hline & Triticum monococcum & bases glumes & 41 & * & * \\
\hline \multirow[t]{9}{*}{ Flore sauvage } & Chenopodium album & & 1 & $*$ & $*$ \\
\hline & Corylus avellana & & 1 & 1 & 2 \\
\hline & Fallopia convolvulus & & 168 & $*$ & $*$ \\
\hline & Lapsana communis & & 35 & * & $*$ \\
\hline & Poa annua & & 3 & * & $*$ \\
\hline & Poaceae & & 8 & * & $*$ \\
\hline & Indéterminés & & 22 & $*$ & $*$ \\
\hline & Abdomens insectes & & 2 & $*$ & $*$ \\
\hline & Nombre total de restes & & 7744 & 1 & 653 \\
\hline
\end{tabular}

Tableau 7 : Décomptes des macrorestes du Néolithique moyen II de Démouville « ZAC du Clos Neuf ».

Table 7: Middle Neolithic macroremains from Démouville "ZAC du Clos Neuf".

l'orge vêtue, variété dont il est question à Démouville, elle se cantonne, au Rubané, à l'est du Rhin (Marinval, 1993). Ce n'est qu'au Post-Rubané que cette orge se développe dans les régions situées à l'ouest du fleuve (Heim, 1983; Bakels, 1990). Nous en avons identifié sur le site VSG de Trosly-Breuil (Aisne) [Dietsch-Sellami, 2001]. La situation géographique des sites bas-normands, Démouville et Colombelles, très à l'ouest des sites où l'orge était attestée jusqu'à présent, confere à sa découverte un caractère tout à fait novateur pour le Rubané. Dotée d'une fréquence élevée à Colombelles, elle apparaît comme la céréale prédominante au sein de la fosse 26 de Démouville, confirmant son importance aux yeux des populations rubanées de la région.

La bonne représentation du blé nu - rappelons qu'il constitue $10 \%$ des restes céréaliers - conforte les remarques suscitées par sa présence, plus ténue, à Colombelles. Sa mise en culture semble désormais bien ancrée dès la fin du Rubané, non seulement dans l'est du pays, comme l'a montré L. Berrio (2012) sur le site de Marainville-surMadon "Sous le chemin de Naviot» (Vosges), mais aussi dans l'Ouest comme l'attestent désormais les séries de Colombelles et de manière encore plus nette de Démouville. En l'absence de fragments de rachis, il n'est pas possible d'affirmer que le blé mis en culture à Démouville est, comme à Marainville-sur-Madon, Colombelles et dans les contextes cardiaux (Marinval, 1988 et 1990), de type hexaploïde (Triticum aestivum l.s.). L'hypothèse de l'introduction de cette céréale en Europe nord-occidentale par voie de diffusion méditerranéenne, puis par des contacts entre cardiaux et rubanés sur le territoire de la France actuelle, s'est vue confortée par les travaux de L. Berrio (2012). L'existence de contacts entre les populations cardiales de la péninsule Ibérique et celles de Colombelles est fortement suggérée par la présence conjointe à Colombelles d'une perle en variscite et de ce blé nu auxquels les agriculteurs 


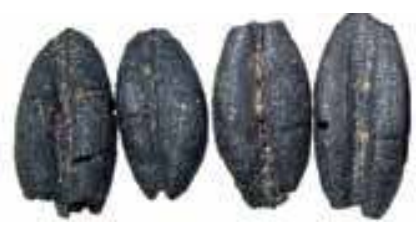

1

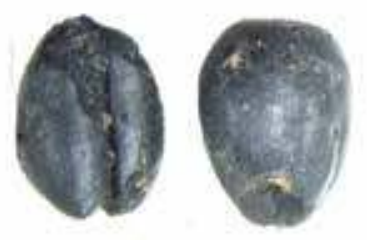

5

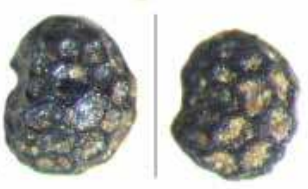

8

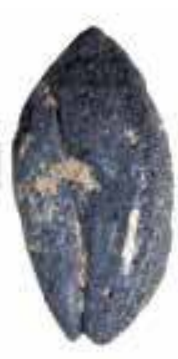

2

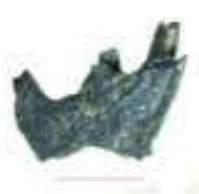

6

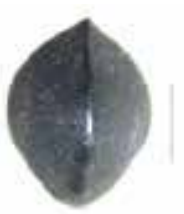

9

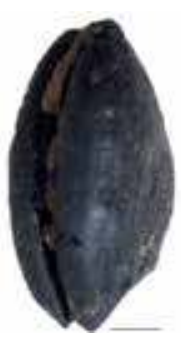

3

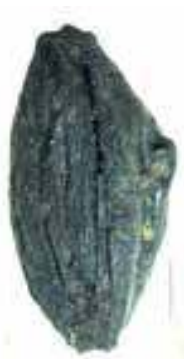

4

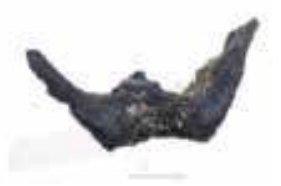

7

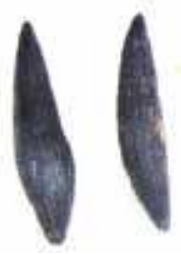

10

Figure 23 : Semences carbonisées du site de Démouville « ZAC du Clos Neuf »(Calvados).

Figure 23: Carbonized seeds from the archaeological site of Démouville "ZAC du Clos Neuf” (Calvados).

Caryopses d'orge vêtue (Hordeum vulgare) ( $n^{\circ} 1$ ), d'amidonnier (Triticum dicoccum) ( $\left.n^{\circ} 2\right)$, d'engrain (Triticum monococcum) ( ${ }^{\circ} 3$ et 4 , ce dernier encore enveloppé de sa balle), de blé nu (Triticum aestivum/turgidum/durum) ( $\left.n^{\circ} 5\right)$; furcas d'engrain ( $n^{\circ} 6$ ) et d'amidonnier $\left(n^{\circ} 7\right)$; semences de pavot somnifère (Papaver somniferum) ( $\left.n^{\circ} 8\right)$, de renouée-liseron (Fallopia convolvulus) ( $n^{\circ} 9$ ) et de lampsane commune (Lapsana communis) ( $\left.n^{\circ} 10\right)$. Toutes les semences proviennent de la fosse rubanée 26, à l'exception de la furca d'amidonnier, issue de la fosse 24 (NMII). Chaque échelle représente $1 \mathrm{~mm}$.

Barley (Hordeum vulgare) ( $n^{\circ} 1$ ), emmer (Triticum dicoccum) ( $\left.n^{\circ} 2\right)$, einkorn (Triticum monococcum) ( $n^{\circ} 3$ et 4 , the latter remaining enclosed in its glume), naked wheat (Triticum aestivum/turgidum/durum) ( $n^{\circ}$ 5); spikelet forks of einkorn ( $\left.n^{\circ} 6\right)$ and emmer ( $\left.n^{\circ} 7\right)$; seeds of opium poppy (Papaver somniferum) ( $\left.n^{\circ} 8\right)$, blackbindweed (Fallopia convolvulus) ( $n^{\circ}$ g) and nipplewort (Lapsana communis) ( $n^{\circ}$ 10). All the macroremains are issued from the Bandkeramik pit 26, except for the spikelet fork of emmer issued from the pit 24 (Middle Neolithic II). Each scale represents $1 \mathrm{~mm}$.

Clichés M.F. Dietsch-Sellami. @ Inrap / Photos M.F. Dietsch-Sellami @ Inrap.

cardiaux de la péninsule Ibérique, tels ceux de La Draga en Catalogne (Buxó et Piqué, 2008), de La Coveta de l'Or et de la Cueva de los Murcielagos (Hopf, 1988; Zohary et Hopf, 2000) accordent une place de choix.

\section{Les macrorestes végétaux associés à l'occupation du Néolithique moyen II}

Les fosses 11, 24, 34 et 67 relèvent de cette période d'occupation du site. Ces quatre structures livrent dernières est très limitée : dans le premier cas (st. 11), elle se résume à un fragment de coquille de noisette (Corylus avellana), dans le second (st. 67) à un unique grain d'orge nue (Hordeum vulgare var. nudum).

Les taxons attestés au sein des deux autres fosses sont au nombre de neuf dont quatre plantes cultivées. Il s'agit uniquement de céréales, deux à grains nus, l'orge, dont la variété est reconnaissable aux ridules transversales caractéristiques visibles en surface de ses grains, et un blé (Triticum aestivum/turgidum/durum), deux à grains vêtus, les blés amidonniers (Triticum dicoccum, fig. 23, 


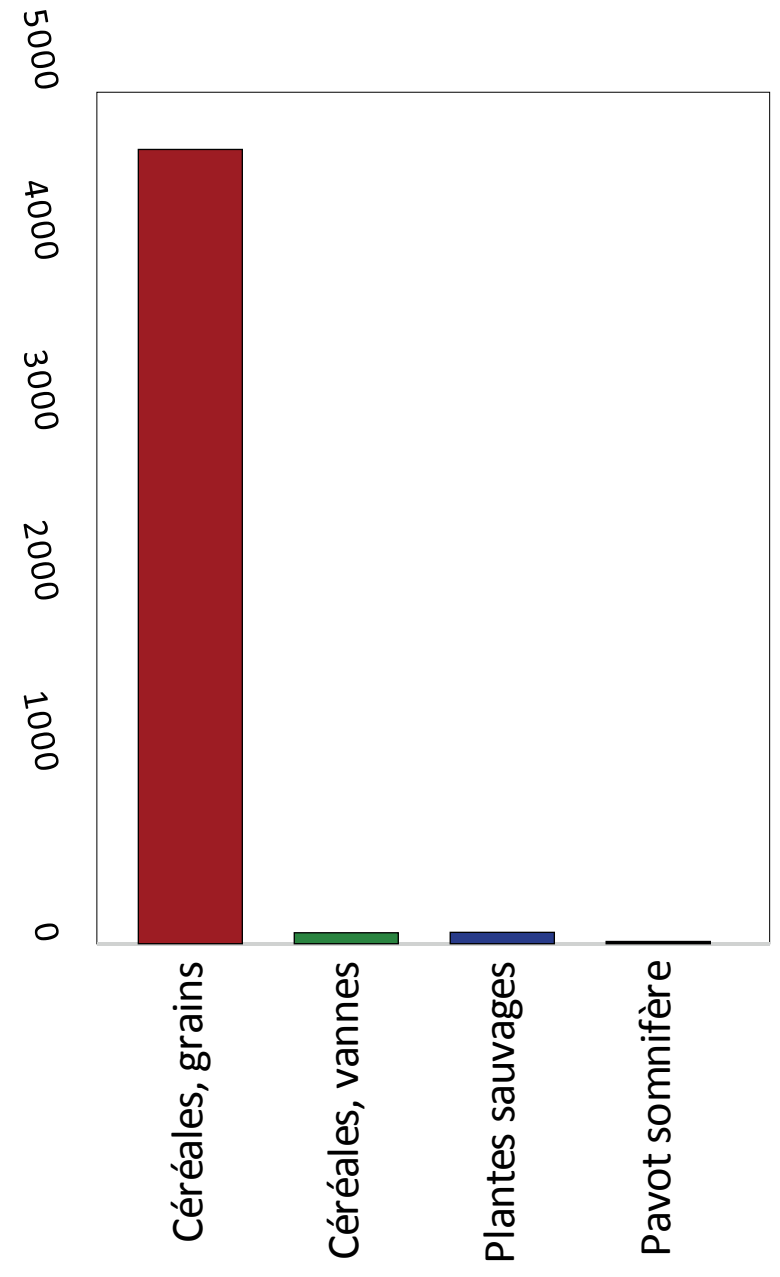

Figure 24 : Représentation relative des différentes catégories de restes végétaux au sein de la fosse rubanée 26 de Démouville «ZAC du Clos Neuf » (Calvados).

Figure 24: Relative distribution of different categories of plant remains in the Bandkeramik pit 26 of Démouville "ZAC du Clos Neuf" (Calvados).

$\mathrm{n}^{\circ} 7$ ) et engrain (Triticum monococcum). Les cinq herbacées sauvages qui complètent ce corpus sont le chénopode blanc (Chenopodium album), la renouée liseron (Fallopia convolvulus), la lampsane commune (Lapsana communis), le pâturin annuel (Poa annua) et des graminées indéterminées (Poaceae).

La fosse 11 livre 653 restes, soit $8 \%$ du corpus. La composition de l'assemblage issu de cette fosse, presque exclusivement formée de grains de céréales (deux fragments de coquilles de noisette sont les seules exceptions notables), peut s'expliquer par l'absence du refus de flottation le plus fin, nous privant d'éventuels sous-produits du traitement des récoltes (vannes, herbacées sauvages). Le caractère vraisemblablement incomplet de cet assemblage ne nous autorise pas à évaluer correctement la représentation des différentes céréales, non plus qu'à comprendre dans quelles circonstances et à quel stade de leur traitement elles ont brûlé. Sur la base de leurs seuls

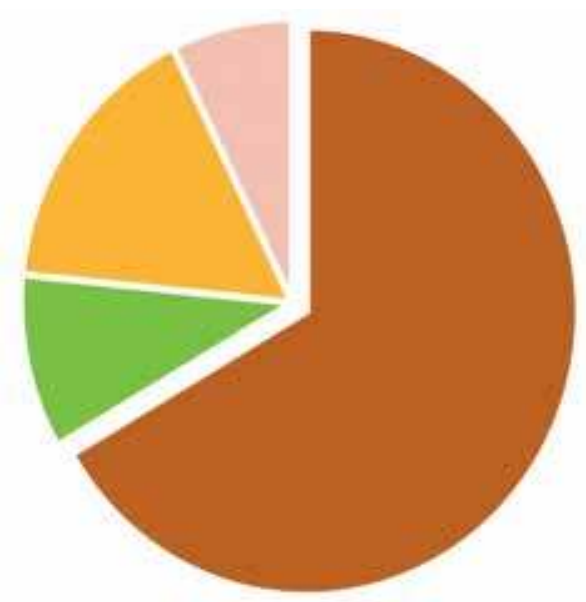

Triticum aestivum/turgidum/durum

\section{Triticum monococcum}

\section{Hordeum vulgare}

\section{Triticum dicoccum}

Figure 25: Représentation des différentes céréales (grains et vannes) dans l'assemblage de la fosse rubanée 26 de Démouville «ZAC du Clos Neuf »(Calvados).

Figure 25: Relative distribution of the different cereals (seeds and chaff) in the assemblage of Bandkeramik pit 26 of Démouville "ZAC du Clos Neuf” (Calvados).

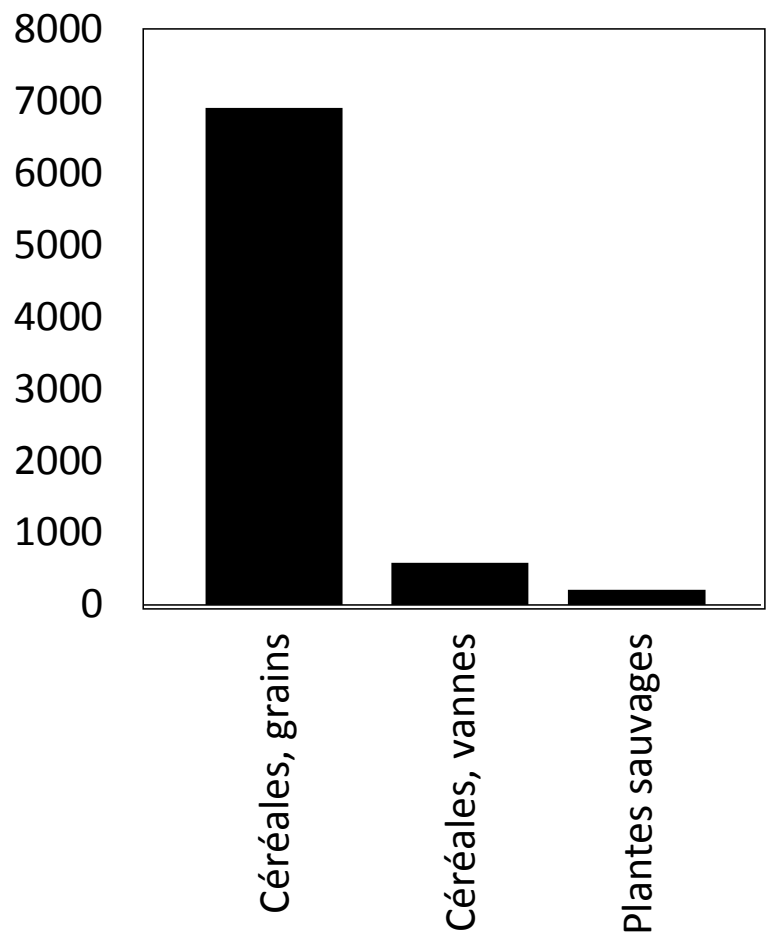

Figure 26 : Représentation relative des différentes catégories de restes végétaux au sein de la fosse NMII 24 de Démouville «ZAC du Clos Neuf ».

Figure 26: Relative distribution of botanical remains in the feature NMII St. 24 at Démouville "ZAC du Clos Neuf" (Calvados). 
grains, les céréales nues - orge et blé - sont nettement prédominantes, formant respectivement $65 \%$ et $30 \%$ de l'assemblage, contre $5 \%$ aux deux blés vêtus. La prépondérance de l'orge nue rejoint les observations formulées à propos des silos du Néolithique moyen II de Mondeville (Dietsch-Sellami, 2006) et plus largement à propos de cette phase du Néolithique dans la moitié nord de la France (Dietsch-Sellami, 2004; Hamon et al., 2019).

En dernier lieu, l'assemblage de la fosse 24, riche de 7744 macrorestes, comporte essentiellement des grains des céréales précédemment mentionnées (fig. 26). Sa composition nous amène à démentir ce qui vient d'être dit sur l'importance des céréales à grains nus au Néolithique moyen II (et à souligner la nécessité de travailler sur les fréquences des plantes cultivées sur un même site et dans un cadre géographique élargi et donc de procéder en amont à un échantillonnage aussi exhaustif que possible!), dans la mesure où les blés vêtus, en particulier l'amidonnier, y sont nettement mieux représentés que les céréales à grains nus. Le corpus céréalier compte $63 \%$ d'amidonnier, $5 \%$ d'engrain dont on notera au passage l'assez bonne performance d'orge nue $(30 \%)$ et seulement $1 \%$ de blé nu. Ici encore, l'orge nue semble favorisée par rapport au blé nu (fig. 27). Ces céréales à grains nus semblent nettoyées (seuls deux rachis de blé nu ont été dénombrés),

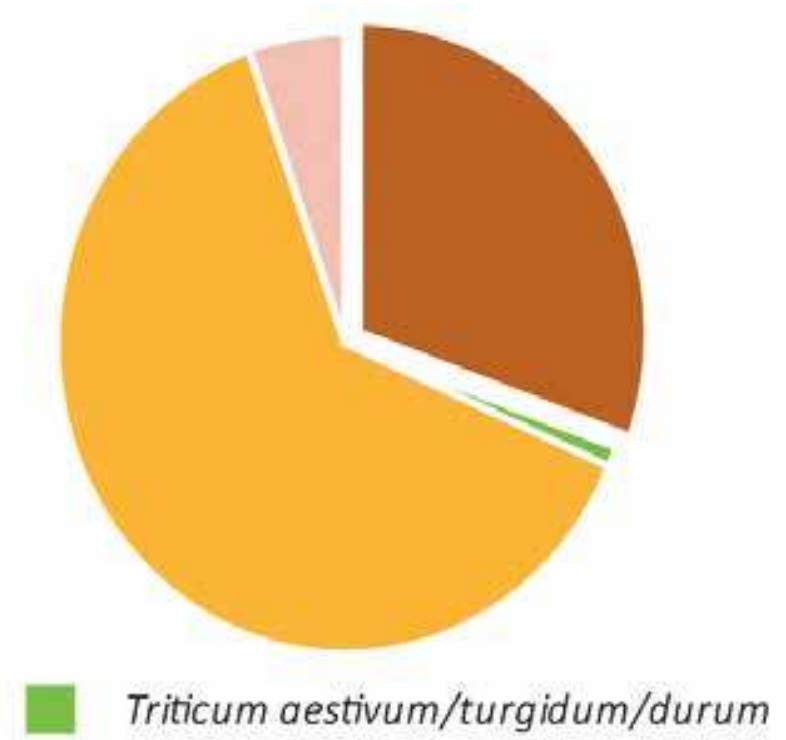

Triticum monococcum

Hordeum vulgare

\section{Triticum dicoccum}

Figure 27 : Représentation des différentes céréales (grains et vannes) dans l'assemblage de la fosse NMII 24 de Démouville « ZAC du Clos Neuf ».

Figure 27: Relative distribution of the different cereals (seeds and chaff) in the assemblage of pit 24 (Middle Neolithic II) of Démouville "ZAC du Clos Neuf" (Calvados). tandis que les sous-produits du traitement des blés vêtus, ajoutés aux semences d'herbacées sauvages, représentent environ $10 \%$ de l'assemblage (fig. 26).

La composition de cet assemblage, mêlant des céréales qui ne requièrent pas les mêmes traitements et dont certaines apparaissent moins nettoyées que d'autres, est le reflet de différents processus antérieurement à leur rejet dans la fosse. Parmi ces activités domestiques, il en est peut-être une qui concerne cette fois une des herbacées sauvages attestées, la renouée-liseron (Fallopia convolvulus). La forte représentation des akènes de cette renouée (fig. 28), dont le NMI est évalué à 168, et la proportion des semences qu'ils représentent parmi les plantes sauvages $(77,7 \%)$, suggèrent que leur présence n'est peut-être pas seulement liée à leur seul statut d'adventice des récoltes. Sans pouvoir en apporter la preuve absolue, il n'est pas exclu que cette plante ait été utilisée par les populations du Néolithique moyen II de Démouville pour les qualités gustatives que leur attribue F. Couplan (1989) - «tendres, farineuses, et [...], même crues, d'un goût agréable exempt d'astringence » et qui font dire à cet auteur qu'elles ont " été mangées en bouillie depuis le Néolithique».

\section{LA CHRONOLOGIE ET LES DATATIONS ${ }^{14} \mathrm{C}$}

L'étude du mobilier et la réalisation de multiples datations par le radiocarbone permet de séparer deux occupations néolithiques distinctes : l'une dans une phase précoce du Néolithique ancien régional, la seconde à la charnière Néolithique moyen I et II ${ }^{7}$.

\section{Le mobilier du Néolithique ancien}

Le lot céramique est assez restreint, mais il permet tout de même quelques observations. Son calage dans une étape finale du RRBP s'appuie sur l'utilisation du peigne à trois dents et celle de bandes incisées hachurées, ces dernières étant particulièrement bien représentées dans cette étape, notamment à Colombelles. Deux éléments contrastent fortement avec ce dernier site : la présence de décors secondaires (totalement absents à Colombelles et qui disparaissent dans le BVSG) et celle de la céramique de type Limbourg (uniquement représentée par deux tessons à Colombelles). Ces différences pourraient être l'indice d'une antériorité de Démouville sur Colombelles.

Concernant l'industrie lithique, la série de Démouville présente des caractères communs à la plupart des sites du Néolithique ancien de la Plaine de Caen, et plus largement, du Bassin parisien, mais également des particularités notables. On retrouve, à l'instar des autres sites de la

7. Cinq de ces datations ont été réalisées dans le cadre du programme Artemis-Culture. 


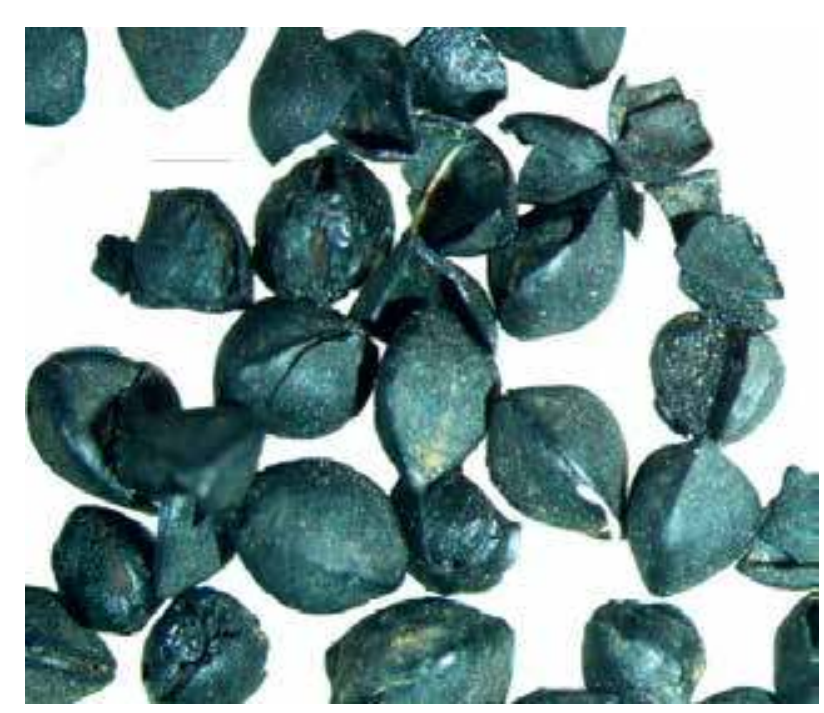

Figure 28 : Akènes de renouée-liseron (Fallopia convolvulus) provenant de la fosse 24 de Démouville « ZAC du Clos Neuf ».

Figure 28: Achenes of black-bindweed (Fallopia convolvulus) issued from pit 24 of Démouville “ZAC du Clos Neuf” (Calvados).

région, une préférence marquée pour l'apport de matières premières de qualité (ici le silex du Cinglais) exploitées pour un débitage laminaire réalisé sur place, sans qu'il s'agisse pour autant d'une production excédentaire. Une production d'éclats est également reconnue : elle met en jeu des savoir-faire moins élevés et s'exerce indifféremment des matériaux reconnus. Ces caractères sont communs à Démouville et Colombelles pour la période Rubanée (Billard et al., 2014), mais également au BVSG, notamment à Verson (Germain-Vallée et al., 2014), et sont partagés par la majorité des sites du Néolithique ancien du Bassin parisien (Bostyn, 1994). En revanche, Démouville présente un panel d'outils qui présente quelques particularités, au premier rang desquelles les burins sur lame, quasiment absents de la série alors qu'ils sont souvent représentés en forte proportion dans les assemblages du Néolithique ancien, y compris à Colombelles (ibid.). De fait, la série présente une forte part d'outils sur éclats, notamment d'outils expédients, à côté desquels les outils sur lame apparaissent minoritaires. La présence d'un pic massif en grès est également rare dans ce type de contexte.

\section{Les datations de l'occupation Néolithique ancien}

Au total, sept datations ont été nécessaires pour la partie néolithique du site (tabl. 8). Elles s'ajoutent aux cinq datations portant sur la nécropole de l'âge du Bronze : l'une d'entre elles a été réalisée en complément de la fouille préventive pour confirmer la datation à l'âge du Bronze d'une tombe n'ayant livré pour tout mobilier funéraire qu'un lot de dentales (sépulture 83).
Les trois datations ${ }^{14} \mathrm{C}$ effectuées sur les structures $\mathrm{du}$ Néolithique ancien sont très proches et couvrent la fin $\mathrm{du} \mathrm{VI}^{\mathrm{e}}$ millénaire, voire le début du premier siècle $\mathrm{du} \mathrm{V}^{\mathrm{e}}$ millénaire, avec pour intervalle commun [52004950] cal. BC. Ces dates sont totalement convergentes avec celles fournies par le site de Colombelles, pour lequel sept échantillons sur treize offrent pour intervalle commun la période [5225-5053] cal. BC.

Cet intervalle, soit les deux derniers siècles du VI ${ }^{\mathrm{e}}$ millénaire, est clairement plus ancien que les dates attribuées au BVSG. On sait toutefois que, pour les sites à la charnière des $\mathrm{VI}^{\mathrm{e}}$ et $\mathrm{V}^{\mathrm{e}}$ millénaires, l'effet plateau a clairement un effet négatif pour cette période, effet qui tend à " tirer » artificiellement les intervalles vers 5200 . Pour le Chasséen méridional, T. Perrin a bien démontré que la phase admise par la plupart des auteurs concernant la phase ancienne du Chasséen méridional (4280-4000 cal. BC) se superpose parfaitement avec l'un des intervalles de plateau de la courbe de calibration (Perrin, 2016). Qu'il s'agisse du Chasséen méridional ou de la fin du Rubané, cet effet statistique apporte un biais important à toute tentative actuelle de séquençage.

Si les datations de Démouville assurent l'antériorité de l'occupation du Néolithique ancien par rapport au BVSG, elles ne permettent pas de la situer chronologiquement par rapport à Colombelles. Elles confirment seulement la précocité de la colonisation rubanée de la Plaine de Caen, en particulier si on les compare à celles obtenues dans d'autres secteurs du Bassin parisien.

\section{Les datations de l'occupation Néolithique moyen}

En faible quantité, le mobilier céramique associé à l'occupation du Néolithique moyen ne permet pas d'affiner la position chronologique. Le mobilier lithique de la phase Néolithique moyen présente également un effectif réduit. Deux petites haches en dolérite sont rattachables à cette phase. Pour le reste, les rares éléments typologiques ne permettent pas une comparaison pertinente avec les autres ensembles de la région.

Trois des quatre datations par le radiocarbone effectuées sur des structures du Néolithique moyen sont regroupées dans l'intervalle [4300-4000] cal. BC, soit le début du Chasséen (tabl. 8). Seule la structure 67 s'en écarte avec un intervalle qui correspond davantage à l'extrême fin du BVSG ou au Cerny : [4767-4541] av. J.-C. cal. Si cette datation est correcte, nous aurions donc un cumul de trois occupations néolithiques sur le même lieu, cas de figure très rarement rencontré, à l'exception du site de Saint-André-sur-Orne (Calvados) qui voit se succéder trois occupations entre le Néolithique récent et le Campaniforme (Ghesquière et al., 2019). 


\section{L'ORGANISATION DES STRUCTURES NÉOLITHIQUES ET LA FONCTION DU SITE}

Cette opération montre toute la difficulté de différencier des structures d'habitat du Néolithique ancien et moyen dans ce type de contexte limoneux, d'autant que l'abondance de céréales est commune aux deux phases d'occupation. Des informations se dégagent néanmoins de cette opération.

Pour l'occupation du Néolithique ancien, le schéma de conservation de type Colombelles ou Verson se reproduit ici, avec notamment une absence totale de trous de poteau observés, les structures se limitant à des fosses de grande taille (fosses latérales et probables silos). L'organisation générale des structures met en évidence deux groupes de structures : au sud, les structures 86 et 174 appartiennent au même alignement et sont peut-être associées aux fosses 68 et 78; au nord, les fosses 26 et 28 forment un deuxième ensemble, auquel sont peut-être associées certaines des fosses voisines non datées. L'occupation du Néolithique ancien pourrait donc se résumer à deux ensembles distants d'environ $60 \mathrm{~m}$ et susceptibles de matérialiser une voire deux unités d'habitation orientées grossièrement est-ouest.

En comparaison du site de Colombelles, la présence des céréales est mieux marquée à Démouville.
Une possible extension du site hors emprise n'est pas impossible, mais les tranchées du diagnostic n'ont livré aucune autre structure néolithique (fig. 1).

L'occupation du Néolithique moyen est plus difficile à cerner et se résume à un minimum de six petites fosses proches les unes des autres où les céréales sont également bien présentes. Les cas de découverte de silos apparemment déconnectés de tout habitat sont relativement fréquents en Plaine de Caen, à moins d'invoquer des problèmes de conservation ou de détection des structures d'habitat. La quantité de mobilier reste toutefois trop peu importante pour assurer la présence d'un habitat à proximité. Il n'en reste pas moins que l'attractivité du site reste intacte entre la fin du Rubané et le début du Chasséen.

\section{CONCLUSION : DÉMOUVILLE ET LA COLONISATION AGRICOLE NORMANDE}

Démouville rejoint le petit groupe des sites de ce Néolithique très ancien, sites qui contribuent à ancrer l'image d'une colonisation agricole précoce en Plaine de Caen : Colombelles "Le Lazzaro " en premier lieu, mais aussi Moult "Le Relais de Poste " (Ghesquière et al., 2018). Ce dernier site découvert dans la plaine alluviale de la Dives, au pied de la cuesta du Pays d'Auge, a fourni

\begin{tabular}{|c|c|c|c|c|}
\hline $\begin{array}{l}\text { Référence } \\
\text { datation }\end{array}$ & $\begin{array}{l}\text { Référence } \\
\text { échantillon }\end{array}$ & Contexte échantillon & Matériau & Résultat \\
\hline $\begin{array}{l}\text { Lyon-7798 } \\
\text { (GrA) }\end{array}$ & $\begin{array}{l}\text { st. 86, US } \\
161.7(21)\end{array}$ & Fosse Néolithique ancien & $\begin{array}{l}\text { Noisette } \\
\text { carbonisée }\end{array}$ & 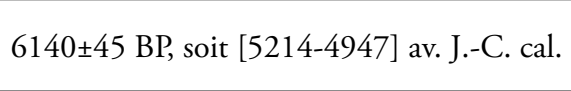 \\
\hline $\begin{array}{c}\text { Ly-8302 (SacA } \\
25487)\end{array}$ & st. 174 & Fosse Néolithique ancien & $\begin{array}{c}\text { Noisette ou } \\
\text { noyau carbonisé }\end{array}$ & 6120 \pm 35 BP, soit [5207-4947] av. J.-C. cal. \\
\hline Ly-7800 (GrA) & $\begin{array}{c}\text { st. 26, US 75-1 } \\
(6)\end{array}$ & Fosse Néolithique ancien & Carporestes & 6075 \pm 45 BP, soit [5201-4847] av. J.-C. cal. \\
\hline Ly-7799 (GrA) & $\begin{array}{c}\text { st. } 34 \text {, US } 56 \\
(4)\end{array}$ & Fosse Néolithique moyen & $\begin{array}{l}\text { Charbon de } \\
\text { bois }\end{array}$ & $5420 \pm 50$ BP, soit [4350-4080] av. J.-C. cal. \\
\hline $\begin{array}{l}\text { Lyon-13041 } \\
\text { (SacA 46618) }\end{array}$ & $\begin{array}{c}\text { St. } 67 \text {, US } 208- \\
\text { 1, fait } 30\end{array}$ & Fosse Néolithique moyen & Céréale & $5795 \pm 40 \mathrm{~PB}$, soit $[4767-4541]$ av. J.-C. cal. \\
\hline $\begin{array}{l}\text { Lyon-13041 } \\
\text { (SacA 46618) }\end{array}$ & $\begin{array}{l}\text { St. 24, US 28-1, } \\
\text { fait } 3\end{array}$ & Fosse Néolithique moyen & Céréale & 5315 \pm 40 BP, soit [4311-4004] av. J.-C. cal. \\
\hline $\begin{array}{c}\text { Ly-15808 } \\
(\text { SacA-55295) }\end{array}$ & St.11, US 20-1 & Fosse Néolithique moyen & Céréale & 5305 \pm 30 BP, soit [4235-4045] av. J.-C. cal. \\
\hline Beta-281973 & st. $97 /$ sq. 113 & Sépulture nécropole & Os & $3060 \pm 40$ BP, soit [1420-1220] av. J.-C. cal. \\
\hline Beta-281974 & st. $112 /$ sq. 177 & Sépulture nécropole & Os & $3370 \pm 40$ BP, soit [1750-1530] av. J.-C. cal. \\
\hline Beta-281975 & st. $88 /$ sq. 541 & Sépulture nécropole & Os & 3220 40 BP, soit [1600-1420] av. J.-C. cal. \\
\hline Lyon-7801 & st. $175 /$ sq. 369 & Sépulture nécropole & Os & $3430 \pm 40$ BP, soit [1877-1631] av. J.-C. cal. \\
\hline $\begin{array}{c}\text { Ly-1 } 1719 \text { (SacA } \\
39748)\end{array}$ & st. $83 /$ sq. 224 & Sépulture nécropole & Os & 3330 \pm 30 BP, soit [1689-1528] av. J.-C. cal. \\
\hline
\end{tabular}

Tableau 8: Résultats des datations par radiocarbone du site de Démouville « ZAC du Clos Neuf ». 
un lot de mobilier caractérisé par l'utilisation du peigne à deux dents et une bonne représentation de la céramique de type Limbourg.

Un tesson à motif de décor imprimé en $\mathrm{T}$ a également été signalé par B. Edeine sur le site de La Brèche-auDiable à Soumont-Saint-Quentin (Calvados) [Edeine, 1965a et b, 1970, 1972; Desloges, Ghesquière, Marcigny, 2011, fig. 7].

Un simple regard sur la situation des sites mentionnés - Colombelles, Démouville, Moult et SoumontSaint-Quentin en incluant Fontenay-le-Marmion "La Hoguette " - permet d'observer que les quatre sites considérés sont tous situés dans une zone géographique restreinte de la Plaine de Caen, à l'est du cours de l'Orne. La localisation de ce groupe de sites à plus de $200 \mathrm{~km}$ à l'ouest des sites rubanés les plus occidentaux, tels que ceux de la basse-vallée de la Marne (Lanchon, 2008) ou ceux de l'Oise tels que Chambly (Boucneau et al., 1996; Herbaut et Martinez, 1997) et de Pont-Sainte-Maxence (Prodeo et al., 1997) ne pose plus de problème d'interprétation dans la mesure où des découvertes récentes ont permis de combler les vides intermédiaires, comme à Saint-Pierred'Autils (Eure) ou à Sours (Eure-et-Loir). Dans la basse vallée de la Seine, le site de la carrière GSM à Saint-Pierred'Autils (Eure) a livré un mobilier qui semble pouvoir appartenir à l'étape finale du Rubané récent du Bassin parisien (Prost et al., 2012) : on y retrouve le motif de décor en $\mathrm{T}$ ainsi que la présence de décors secondaires.

Géographiquement proche, le site de Sours, en Eure-etLoir, près de Chartres, offre également certains attributs propres au corpus des sites de la fin du Rubané (Dupont, Liagre et Irribaria, 2010), notamment le motif de décor en $\mathrm{T}$ avec un décor secondaire en croix et la présence de céramique Limbourg ou d'inspiration Limbourg.

Par ses datations convergentes avec celles de Colombelles, le site de Démouville assure un bon ancrage chronologique et confirme la présence de la céramique non rubanée de type Limbourg en contexte rubané.

L'identification des céramiques de type Hoguette et Limbourg en contexte rubané constitue un des éléments majeurs du débat sur les modalités de la néolithisation du Bassin parisien. Par le hasard des découvertes, elle touche particulièrement la Normandie, puisque l'un de ces deux faciès céramiques a été défini à partir d'un vase découvert dans le paléosol du tumulus de la Hoguette à Fontenayle-Marmion (Calvados) qui se situe à une dizaine de kilomètres de Colombelles (Caillaud et Lagnel, 1972).

Sans rentrer dans la discussion sur l'origine de cette production céramique, Hoguette et Limbourg constituent probablement deux phénomènes distincts, le premier plutôt associé à des contextes du Rubané ancien et moyen (avec toutefois une datation récente de la céramique
Hoguette d'Alizay (Eure), à la confluence de la Seine et de l'Eure : [5370-5222] cal. av. J.-C.; Ghesquière, 2017). La céramique du Limbourg, particulièrement dans le RRBP de la vallée de l'Aisne, semble plus récente et son influence pourrait se retrouver dans certains styles céramiques $\mathrm{du}$ Bq-VSG.

Le site de Démouville constitue donc un nouveau jalon pour attester d'une colonisation agricole précoce de la Normandie dans une étape finale du RRBP et la présence de céramique de type Limbourg susceptibles d'être des productions de populations autochtones en voie d'acculturation.

La présence de blocs d'hématite confirme également l'intégration précoce de ce matériau dans les réseaux d'échange des pionniers rubanés. La présence d'un grand pic en grès pourrait constituer un indice supplémentaire de l'émergence d'une activité d'extraction minière.

Démouville constitue enfin une référence par l'abondance de macrorestes végétaux découverts. Comme à Colombelles et comme sur les sites rubanés, on y retrouve la présence systématique de l'amidonnier et de l'engrain. À Démouville comme à Colombelles, l'abondance de l'orge vêtue constitue un fait remarquable, une particularité dans le Rubané français, qu'il faudra expliquer.

La culture du blé nu, bien attestée à Démouville, vient à nouveau alimenter le débat d'une possible introduction de cette céréale depuis les régions méditerranéennes et doit être rapprochée de l'hypothèse d'échanges à longue distance avec la sphère cardiale dont témoigne, à Colombelles, la découverte d'une perle en variscite provenant de la péninsule Ibérique.

\section{Bibliographie}

Alix P., Arbogast R. M., Pinard E. et Prodeo F., 1997, Le méandre de Pont-Sainte-Maxence (Oise) au Néolithique ancien, dans Jeunesse C. (dir.), Le Néolithique danubien et ses marges, entre Rhin et Seine, Actes du 22 Colloque interrégional sur le Néolithique (Strasbourg, 1995), Cahiers de l'APRAA, supplément $\mathrm{n}^{\circ} 3$, p. 359-399.

Artur E. et Billard C., avec la collaboration de Hervieu G., Marie N. et Dubrulle F., 2008, "Les occupations du Mésolithique final de Biéville-Beuville "Le Vivier" (Calvados) ", Revue archéologique de l'Ouest, 25, p. 53-92.

Aubry B., Honoré D., Guillon M. et Fromont N., 2010, "Une sépulture du Néolithique ancien à Saint-Pierre-d'Autils "carrière GSM" ", dans Billard C. et Legris M. (dir.), "Premiers Néolithiques de l'Ouest", colloque interrégional sur le Néolithique (Le Havre, 2007), Rennes, PUR, coll. " Archéologie et Culture ", p. 101-116.

Bakels C. C., 1979, "Linearbandkeramische Früchte und Samen aus den Niederlanden ", Archaeo-Physika, 8, p. 1-10. 
Bakels C. C., 1984, "Carbonized seeds from Northern France», Analecta praehistorica Leidensia, 17, p. 1-27.

BAKels C. C., 1990, "The crops of the Rössen culture: significantly diffrent from their bandkeramik predecessors - french influence? ", dans Cahen D. et Отte M. (éd.), Rubané et Cardial, actes du colloque de Liège (novembre 1988), Liège, ERAUL, 39, p. 83-87.

Bakels C. C., 1991, "Western Continental Europe ", dans VaN Zeist, Wasylikowa et Behre (éd.), Progress in Old World Palaeoethnobotany, Rotterdam, Balkema, p. 279-295.

Bakels C. C., 1995a, "Les restes carbonisés de graines et de fruits ", dans Ilett M. et Plateaux M., Le site néolithique de Berry-au-Bac "Le Chemin de la Pêcherie" (Aisne), Monographie du Centre de Recherches Archéologiques, 15, Paris, CNRS éditions, p. 142-143.

Bakels C. C., 1995b, "Bucy-le-Long "La Fosse Tounise”/"La Héronnière" et la continuité Rubané-Villeneuve-Saint-Germain : l'apport de l'analyse des graines carbonisées ", dans Ilett M., Constantin C. et Farrugia J.-P., Bâtiments voisins du Rubané et du groupe de Villeneuve-Saint-Germain sur le site de Bucy-le-Long "La Fosse Tounise " (Aisne), n spécial 9, Revue archéologique de Picardie, p. 37-38.

Bakels C. C. et Rousselle R., 1985. "Restes botaniques et agriculture du Néolithique ancien en Belgique et aux PaysBas", Helinium, XXV, p. 37-57.

Berrio L., 2012, Économie végétale et alimentaire en Lorraine au Néolithique ancien. Étude carpologique du site rubané de Marainville-sur-Madon "Sous le chemin de Naviot" (Vosges), mémoire de master 2 "Archéologie et Environnement", Université Paris I.

Billard C., Bostyn F., Hamon C. et Meunier K., dir., 2014, L'habitat du Néolithique ancien de Colombelles "Le Lazzaro " (Calvados), Paris, Société préhistorique française (Mémoires, 58), $410 \mathrm{p}$.

Billard C., Savary X., Dupret L. et Hamon C., 2016a, « Premières données sur l'exploitation de l'hématite en BasseNormandie durant la préhistoire récente : ses contextes archéologiques et géologiques, son insertion dans le cadre de la néolithisation de l'ouest du Bassin parisien ", dans BILLARD C. et al. (éd.), Autour de l'hématitelAbout haematite, actes de la table ronde de Jambes (7-8 février 2013), Anthropologica et Praehistorica, 125, p. 63-87.

Billard C., Savary X., Bosquet D., Jadin I., Hamon C., Goemaere E., Dreesen R., Dupret L. et Querré G., 2016b, «Différenciation des hématites oolithiques à partir d'observations macroscopiques non destructives : essais de comparaison des matériaux ordoviciens normands et dévoniens belges ", dans Billard C. et al. (éd.), Autour de l'hématitel About haematite, actes de la table ronde de Jambes (7-8 février 2013), Anthropologica et Praehistorica, 125, p. 193-202.

Bostyn F., 1994, Caractérisation des productions et de la diffusion des industries lithiques du groupe néolithique de Villeneuve-Saint-
Germain, thèse de doctorat de l'université de Paris X, 745 p.

Bostyn F., Charraud F. et Denis S., 2019, "Variabilités techniques, évolutions et aires d'influence des centres de productions laminaires au sein de la culture de Blicquy/Villeneuve-Saint-Germain ", dans Montoya C., Fagnart J.-P. et Lоснт J.-L. (dir.), Préhistoire de l'Europe du Nord-Ouest : mobilités, climats et entités culturelles. XXVIII Congrès préhistorique de France (Amiens, 30 mai-4 juin 2016), Paris, Société Préhistorique Française, p. 43-56.

Boucneau N., Bostyn F. et Martinez R., 1996, "Chambly "Le Clos de la Rivière" (Oise) : un site Rubané récent très occidental ", "La Bourgogne entre les bassins rhénan, rhodanien et parisien : carrefour et frontière? ", actes du $18^{e}$ colloque interrégional sur le Néolithique (Dijon, 1991), $14^{\mathrm{e}}$ supplément, Revue archéologique de l'Est, p. 271-294.

Chancerel A., Marcigny C. et Ghesquière E., 2006, Le plateau de Mondeville (Calvados) du Néolithique à l'âge du Bronze, Paris, Éditions de la Maison des Sciences de l'Homme (Document d'Archéologie française, 99), 205 p.

Charraud F., 2015, « Exploitation minière et gestion des lames en silex du Cinglais au Néolithique ancien : de la minière d'Espins (Calvados) "Foupendant" aux habitats du nordouest de la France ", Bulletin de la Société préhistorique française, t. $102, \mathrm{n}^{\circ} 2$, p. 317-338.

Constantin C., 1985, "Fin du Rubané, céramique du Limbourg et post-Rubané, le Néolithique le plus ancien en Bassin parisien et en Hainaut ", Oxford, Bar Publishing (British archaeological reports, 273), 2 vol., 650 p.

Constantin C., 2013, « Précision sur l'étape finale du Rubané récent du Bassin parisien et la formation du groupe de Villeneuve-Saint-Germain ", Chronologie du Rubané dans le Bassin parisien, actes de la séance de la Société préhistorique française (Paris, 10 juin 2011), Paris, Société préhistorique française, Bulletin de la Société préhistorique française, 110, 3, p. 473-494.

Constantin C. et Ilett M., 1997, "Une étape finale dans le Rubané récent du Bassin parisien ", dans Jeunesse C. (dir.), Le Néolithique danubien et ses marges, entre Rhin et Seine, Actes du 22 Colloque interrégional sur le Néolithique (Strasbourg, 1995), Cahiers de l'APRAA, supplément n³, p. 281-300.

Couplan F., 1989, Le régal végétal. Plantes sauvages comestibles. Encyclopédie des plantes comestibles de l'Europe, vol. 1, Flers, Équilibres Aujourd'hui, 453 p.

Desloges J., 1986, “Fouilles de mines à silex sur le site néolithique de Bretteville-le-Rabet (Calvados) ", Actes $d u X^{e}$ colloque interrégional sur le Néolithique (Caen, 1983), supplément $\mathrm{n}^{\circ} 1$, Revue archéologique de l'Ouest, p. 73-101.

Desloges J., Ghesquière E. et Marcigny C., 2010, « La minière Néolithique ancien / moyen I des Longrais à SoumontSaint-Quentin (Calvados) ", Revue archéologique de l'Ouest, 27, p. 21-38.

Dietsch-Sellami M.-F., 2001, Étude carpologique du remplissage de quelques fosses du site néolithique ancien (Villeneuve-Saint- 
Germain) de Trosly-Breuil (Aisne), rapport inédit, Amiens, SRA Picardie.

Dietsch-Sellami M.-F., 2004, « L'alternance céréales à grains vêtus, céréales à grains nus au Néolithique : nouvelles données, premières hypothèses ", Internéo, Actes de la Journée d'information du 20 novembre 2004, Association pour les études interrégionales sur le Néolithique, Paris, Société préhistorique française, p. 125-135.

Dietsch-Sellami M.-F., 2006, "Carpologie », dans Chancerel A., Marcigny C. et Ghesquière E. (dir.), Le plateau de Mondeville (Calvados) du Néolithique à l'âge du Bronze, Paris, Éditions de la Maison des Sciences de l'Homme (Documents d'Archéologie française, 99), p. 80-87.

Dietsch-Sellami M.-F., 2014, «Étude carpologique », dans BiLlard C., Bostyn F., Hamon C. et Meunier K. (dir.), L'habitat du Néolithique ancien de Colombelles "Le Lazzaro " (Calvados), Paris, Société préhistorique française (Mémoire LVIII de la Société préhistorique française, 58), p. 275-279.

Dreesen R., Savary X. et Goemaere E., « Definition, classification and microfacies characteristics of oolitic ironstones, used in the manufacturing of red ochre - a comparative petrographical analysis of Palaeozoic samples from France, Belgium and Germany", dans Billard C. et al. (éd.), Autour de l'hématitelAbout haematite, actes de la table ronde de Jambes (7-8 février 2013), Anthropologica et Praehistorica, 125, p. 203-223.

Dupont F., Liagre J. et Irribaria R., 2010, "Sours "Les Ouches" (Eure-et-Loir), un site original du Villeneuve-SaintGermain ancien en région Centre ", dans Billard C. et LEGRIs M. (dir.), "Premiers Néolithiques de l'Ouest ", colloque interrégional sur le Néolithique (Le Havre, 2007), Rennes, PUR, coll. "Archéologie et Culture ", p. 75-100.

Franchet L. et Giraux L., 1923, Les ateliers néolithiques de la forêt de Montmorency, Bordeaux, $47^{\mathrm{e}}$ session de l'Association française pour l'Avancement des Sciences, p. 642-648.

Fromont N., Charraud F. et Maingaud A., 2013, "Un exemple d'extraction des matériaux calcaires : premiers éléments sur les fosses-carrières et l'outillage du site Néolithique moyen de Saint-Sylvain (Calvados, France) ", dans Guyodo J.-N. et MENs E. (dir.), Les premières architectures en pierre en Europe occidentale, actes du colloque international de Nantes (2-4 octobre 2008), Rennes, PUR, p. 107-122.

Germain-Vallée C., Jan D., Charraud F., Fromont N., Hamon C., Riquier C., Marcoux N., Béguier I. et DietschSellami M.-F., 2019, "Un hameau du Néolithique ancien à Verson (Calvados, Normandie) ", dans Fromont N. et Marchand G. (dir.), Statut des objets, des lieux et des hommes au Néolithique, actes du $32^{e}$ colloque interrégional sur le Néolithique (Le Mans, 24-25 novembre 2017).

Ghesquière E., 2017, Le Mésolithique en Basse-Normandie, Rennes, PUR, coll. "Archéologie et Culture ", 414 p.

Ghesquière E., Charraud F., Giazzon D., Hachem L. et Manceau L., 2019, "Grands bâtiments du Néolithique final à Saint-André-sur-Orne (Calvados) ", dans Montoya C., Fagnart J.-P. et Locht J.-L., Préhistoire de l'Europe du Nord-Ouest : mobilité, climats et identités culturelles, vol. 3, Néolithique - âge du Bronze, Paris, Société préhistorique française, p. 289-306.

Ghesquière E. et Marcigny C., 2014, "Les composantes du paysage et des territoires du Néolithique moyen : autour des enceintes de la plaine de Caen ", dans Joussaume R. et LARGE J.-M. (dir.), Enceintes néolithiques de l'ouest de la France de la Seine à la Gironde, actes du colloque Crabe (19-21 septembre 2012, Les Lucs-sur-Boulogne), Chauvigny, Association des publications chauvinoises (Mémoire XLVIII), p. 255-272.

Ghesquière E., Le Maux N., Lepaumier H. et Marcigny C., 2018, «Un site Rubané/Limbourg en Normandie occidentale : Moult «Le Relais de Poste " (Calvados)». InterNéo, 12 , p. $18-25$.

Ghesquiere E. et Marcigny C., 2011, Cairon. Vivre et mourir au Néolithique, La pierre Tourneresse en Calvados, Rennes, PUR, coll. "Archéologie et Culture ", 200 p.

Goemaere E., Кatsch A., Eschghi I. et Dreesen R., 2016a, "Geological record and depositional setting of Paleozoic oolitic ironstones in Western Europea ", dans Billard C. et al. (éd.), Autour de l'hématite/About haematite, actes de la table ronde de Jambes (7-8 février 2013), Anthropologica et Praehistorica, 125, p. 23-43.

Goemaere E., Salomon H., Billard C., Guerre G., Mathis F., Golitko M., Dubrulle-Brunaud C., Savary X. et DreeSEN R., 2016b, " Les hématites oolithiques du Néolithique ancien et du Mésolithique de Basse-Normandie (France). Caractérisation physico-chimique et recherche des provenances ", dans Billard C. et al. (éd.), Autour de l'hématitel About haematite, actes de la table ronde de Jambes (7-8 février 2013), Anthropologica et Praehistorica, 125, p. 203-223.

Griselin S. avec la collaboration de HAMON C., 2020, Fabrication et fonction des outils de type montmorencien: nouveau regard à partir des découvertes récentes sur les habitats mésolithiques, Paris, Société préhistorique de France (Mémoires, 66), 234 p.

Hamon C., Salavert A., Dietsch-Sellami M.-F. et MonCHABlon C., 2019, "Cultiver et consommer les plantes au Néolithique entre Seine et Meuse : technologie des meules et analyses carpologiques ", dans Montoya C., Fagnart J.-P. et Locht J.-L., Préhistoire de l'Europe du Nord-Ouest : mobilités, climats et entités culturelles, actes du XXVIII colloque de la Société préhistorique française (Amiens, 30 mai-4 juin 2016), vol. 2 : Paléolithique supérieur ancien, Paléolithique final-Mésolithique, Paris, Société préhistorique française, p. 119-137.

Heim J., 1983, "Apports récents de la paléobotanique à la connaissance de l'importance des activités culturales (agricoles) des néolithiques anciens entre Rhin et Seine ", dans DE LaEt S. J. (éd.), Progrès récents dans l'étude du Néolithique ancien, Actes du colloque international de Gand (21-22 mai 1982), Dissertationes Archaeologicae Gandenses, XXI, p. 62-70. 
Heim J., 1985, "Recherches sur l'environnement paléobotanique du village rubané de Darion par l'étude des pollens et des restes de diaspores (graines) ", Bulletin de la Société royale belge d'Anthropologie et de Préhistoire, 96, p. 31-48.

Heiм J., 1988, "Concentration de graines carbonisées dans une fosse danubienne à Crisnée "La Mai" ", Helinium, XXVIII, p. 51-58.

Herbaut F. et Martinez R., 1997, «L'habitat Rubané final de Chambly "Le Clos de la Rivière"(Oise) ", Revue archéologique de Picardie, vol. 3, 3-4, p. 9-28.

Ilett M. et Meunier K., 2013, " Avant-propos ", Chronologie du Rubané dans le Bassin parisien, Actes de la séance de la Société prébistorique française (Paris, 10 juin 2011), Bulletin de la Société préhistorique française, 110, 3, p. 414-420.

Joseph P., 1982, Le minerai de fer ordovicien du Massif armoricain : sédimentologie et paléogéographie, Paris, thèse de l'école nationale supérieur des mines, $352 \mathrm{p}$.

Le Saint-Allain M. avec la collaboration de Dylewski M., Boyle A., Bouvier S., Prat B., Charraud F., Michel J., Delalande A. et Bailliot M., 2013, Démouville, ZAC du Clos Neuf (Calvados), opération de fouille archéologique préventive, rapport final d'opération, Oxford Archaeology, 2 t., 254 p. et $198 \mathrm{p}$.

Le Saint-Allain M., 2013, « La nécropole du Bronze moyen de la "ZAC du Clos Neuf" à Démouville (Calvados) ", Bulletin de l'APRAB, 11, p. 41-44.

Le Roux C.-T., 1999, L'outillage de pierre polie en métadolérite du type A. Les ateliers de Plussulien (Côtes-d'Armor) : production et diffusion au Néolithique dans la France de l'Ouest et audelà, Travaux du laboratoire "Anthropologie, Préhistoire et Quaternaire Armoricains ", Unité mixte de Recherche 6566 "Civilisations atlantiques et Archéosciences ", n 43, Rennes, Université Rennes 1, 244 p.

Lethrosne H., Irribarria R., Creusillet M.-F. et ChaMAux G., 2016, "Quel(s) Chasséen(s) en région Centre-Val de Loire? État des lieux ", dans Perrin T., Chambon P., Gibaja J.-F. et Goude G. (dir.), Le Chasséen, des Chasséens... Retour sur une culture nationale et ses parallèles: Sepulcres de fossa, Cortaillod, Lagozza, Actes du colloque international tenu à Paris (France) du 18 au 20 novembre 2014, Toulouse, Archives d'Écologie Préhistorique, p. 91-122.

MaRINVAL P., 1988, Cueillette, agriculture et alimentation végétale de l'Épipaléolithique jusqu'au deuxième âge du fer en France méridionale, thèse doctorale de l'EHESS.

Marinval P., 1990, «Relations Cardial-Rubané, les apports de la carpologie ", dans Cahen D. et Otтe M. (dir.), "Rubané et Cardial ", Actes du colloque de Liège, novembre 1988, Liège, ERAUL, 39, p. 401-404.

Marinval P., 1993, "Données récentes sur l'agriculture au Néolithique ancien et moyen dans le Nord de la France ", "Le Néolithique au quotidien ", actes du XVI" colloque interrégional sur le Néolithique, Paris, Éditions de la Maison des
Sciences de l'Homme, Documents d'Archéologie Française, 39, p. 122-126.

MARTINEZ R., 1991, "Chasséen septentrional ou néolithique moyen régional ", dans Beeching A., Binder D., BlanChet J.-C., Constantin C., Dubouloz J., Martinez R., Mordant D., Thévenot J.-P. et VAquer J. (dir.), Identité du Chasséen, acte du colloque international de Nemours du 17 au 19 mai 1989, Nemours, Association Pour la Promotion de la Recherche Archéologique en Île-de-France, p. 177-198.

Meunier K., 2012, «Styles céramiques et néolithisation dans le Sud-Est du Bassin parisien ", Recherches archéologiques, $\mathrm{n}^{\circ} 5$, Paris, CNRS Éditions, 276 p.

Meunier K., 2013a, « La céramique de Juvigny "les Grands Traquiers" (Marne) et le Rubané récent champenois ", Chronologie du Rubané dans le Bassin parisien, Actes de la séance de la Société préhistorique française (Paris, 10 juin 2011), Bulletin de la Société préhistorique française, 110, 3, p. 421-446.

Perrin T., 2016, « Le délicat séquençage du Chasséen méridional ", dans Perrin T., Chambon P., Gibaja J.-F. et Goude G. (dir.), Le Chasséen, des Chasséens... Retour sur une culture nationale et ses paralleles: Sepulcres de fossa, Cortaillod, Lagoz$z a$, Actes du colloque international tenu à Paris (France) du 18 au 20 novembre 2014, Toulouse, Archives d'Écologie Préhistorique, p. 437-455.

Prodeo F., Pinard E., Arbogast R.-M. et Alix P., 1997, « Un habitat du Rubané Récent du Bassin Parisien à Pont-SainteMaxence "Le Jonquoire 2" (Oise), dans Jeunesse C. (dir.), Le Néolithique danubien et ses marges, entre Rhin et Seine, Actes $d u 22^{\circ}$ Colloque interrégional sur le Néolithique (Strasbourg, 1995), Cahiers de l'APRAA, supplément nº 3, p. 359-399.

Prost D., Bedault L., Biard M., Dupont C., Fromont N. et Hamon C., 2012, « Le site du Néolithique ancien de SaintPierre-d'Autils (Haute-Normandie-Eure) : présentation liminaire », InterNéo, 9, p. 49-59.

Rioult M., Coutard J.-P., Helluin M., Pellerin J., Quinejure-Helluin E., Larsonneur C. et Alain Y., 1989, Carte géologique de la France (1/50 000), feuille Caen, Orléans, Bureau des ressources géologiques et minières.

Rioult M., Coutard J.-P., de la Querière P., Helluin M., Pellerin J., Larsonneur C., Provost M., 1989, Notice explicative, carte géologique de la France (1/50 000), feuille Caen, Orléans, Bureau des ressources géologiques et minières, $104 \mathrm{p}$.

Tarrête J., 1977, Le Montmorencien, Gallia Préhistoire, suppl. $\mathrm{n}^{\circ} 10$, Paris, CNRS Éditions.

VAN Berg P.-L., 1990, "Céramique du Limbourg et néolithisation en Europe du Nord-Ouest ", dans Cahen D. et Otte M. (dir.), Rubané et Cardial, actes du colloque de Liège (1988), Liège, Études et recherches archéologiques de l'université de Liège, 39, p. 161-208.

Zohary D. et Hopf M., 2000, Domestication of Plants in the Old World, Oxford, Oxford University Press. 
Neben einem bronzezeitlichen Gräberfeld hat die am Fundplatz von Démouville "ZAC du Clos Neuf " (Calvados, Normandie) durchgeführte Rettungsgrabung verschiedene Befunde des Neolithikums zu Tage gefördert, bei denen es sich überwiegend um Vorratsgruben gehandelt haben dürfte. Die Fundanalyse und die Radiokarbondatierungen haben es ermöglicht, zwei verschiedene Siedlungsphasen voneinander abzugrenzen, von denen eine dem Beginn des Frühneolithikums und die andere dem Mittelneolithikum (Néolithique moyen) zuzuweisen ist.

Obwohl es nicht sehr umfangreich ist, hat das keramische Fundmaterial der frühneolithischen Besiedlung von Démouville gestattet, die ältesten Befunde einer kleinen Gruppe von Fundplätzen in der Normandie zuzuordnen, die der Spätphase der Bandkeramik des Pariser Beckens (Rubané récent du Bassin parisien; RRBP) angehören. Durch die Übereinstimmung der Datierungen mit jenen des Fundplatzes von Colombelles bestätigen die Ergebnisse von Démouville die chronologische Einstufung dieser Pionierphase frühneolithischer Besiedlung sowie das Auftreten von nichtbandkeramischen Keramikfunden vom Typ Limburg an bandkeramischen Fundstellen in der Normandie. Die Entdeckung von zwei Hämatitblöcken sowie von einer großen, aus Sandstein gefertigten Hacke bestätigt die frühe Nutzung dieses zum Färben verwendeten Minerals im Rahmen eines Netzwerkes zum Warenaustausch der bandkeramischen Pioniere. Diese Funde stellen starke Indizien für eine Entwicklung eines unterirdischen Gesteinsabbaus dar.

Die frühneolithischen Befunde werden hier von einer Gruppe von mindestens sechs Gruben des Mittelneolithikums begleitet, die einem Fundplatztyp entsprechen, wie er in der Ebene von Caen nicht selten ist und der wohl vorrangig der Verarbeitung und der Speicherung der Ernteerträge diente. Der Fundplatz von Démouville ist ferner durch die große Zahl der dort für beide Perioden des Neolithikums nachgewiesenen botanischen Makroreste besonders bemerkenswert. Was das Frühneolithikum betrift, belegt er ein für die bandkeramische Landwirtschaft typisches Artenspektrum, jedoch hebt er sich wie der Fundplatz von Colombelles durch die Häufigkeit des Auftretens von Spelzgerste hervor, die bisher ausschließlich in bandkeramischen Befunden östlich des Rheins nachgewiesen wurde. Schließlich stimuliert der nachgewiesene Anbau von Nacktweizen erneut die Diskussion einer möglichen Einführung dieses Getreides aus dem Mittelmeergebiet über frühe Fernhandelsbeziehungen mit der Cardial-oder Impressokultur. Für das Mittelneolithikum stimmt das Vorherrschen der Nacktgerste und ganz allgemein von freidreschendem Getreide, mit den Ergebnissen und Beobachtungen von anderen zeitgleichen Fundstellen im nördlichen Frankreich überein.

Schlagwortindex: Démouville, Normandie, Neolithikum, Bandkeramik, Getreide, botanische makroreste.

\section{Las ocupaciones neolíticas de Démouville "ZAC du Clos Neuf »(Calvados) y los inicios de la colonización agrícola en Normandía}

Además de una necrópolis de la Edad de Bronce, la excavación preventiva del yacimiento de Démouville "ZAC du Clos Neuf" (Calvados) ha sacado a la luz estructuras neoliticas, una buena parte de las cuales podrian corresponder a estructuras de almacenamiento. El análisis del material y las fechas de radiocarbono han permitido identificar dos ocupaciones principales, una a principios del Neolítico antiguo y la segunda en el Neolitico medio. El material cerámico del Neolítico antiguo de Démouville, aunque escaso, permite vincular las estructuras más antiguas a un pequeño grupo de yacimientos normandos atribuidos a una etapa final del RRBP (Cultura de la cerámica de bandas reciente de la cuenca de París). Por sus fechas convergentes con las de Colombelles, el yacimiento de Démouville garantiza un buen anclaje cronológico a esta fase pionera y confirma la presencia de cerámica de tradición diferente, de tipo Limburgo, en el contexto de la cerámica de bandas de Normandía. El descubrimiento de 2 bloques de hematita y un gran pico de arenisca también confirma la integración temprana de este material colorante en las redes de intercambio de los pioneros de la cerámica de bandas y puede constituir un indicio importante del surgimiento de una actividad de extracción de minerales.

Las estructuras del Neolitico antiguo están asociadas a un grupo de al menos 6 hoyos del Neolítico medio que forman un tipo de yacimiento recurrente en la llanura de Caen y que parecen dedicados al procesamiento y almacenaje de las cosechas.

Démouville constituye finalmente un punto de referencia por la abundancia de macro-restos botánicos descubiertos (para los dos periodos de ocupación neolíticos). Para el Neolítico antiguo, si el yacimiento encaja bien en las prácticas agrarias de la cerámica de bandas, se distingue (como Colombelles), por la abundancia de cebada vestida, que sólo se observa en los contextos de cerámica de bandas al este del Rin. El cultivo de trigo desnudo alimenta una vez más el debate sobre una posible introducción de este cereal desde el Mediterráneo y los primeros intercambios a larga distancia con la esfera cardial. Para el Neolítico medio, la preponderancia de la cebada desnuda, y en general de los cereales desnudos, coincide con las observaciones realizadas sobre los contextos de la mitad norte de Francia.

Palabras clave: Démouville, Normandía, Neolitico, cerámica de bandas, semillas, restos vegetales. 
